\title{
Calibração Entrópica da Superfície de Volatilidade em Mercados Incompletos
}

\author{
Marcelo Magalhães Taddeo
}

TESE APRESENTADA

$\mathrm{AO}$

INSTITUTO DE MATEMÁTICA E ESTATÍ́STICA PARA

OBTENÇÃO DO GRAU DE MESTRE

EM

MATEMÁTICA APLICADA

\begin{abstract}
Área de Concentração: Matemática Aplicada
Orientador: Prof. Dr. Pedro Paulo Schirmer
\end{abstract}

Durante a elaboração deste trabalho o autor recebeu apoio financeiro da $\mathrm{CNPq}$

São Paulo, março de 2003 


\title{
Calibração Entrópica da Superfície de Volatilidade em Mercados Incompletos
}

\author{
Este exemplar corresponde à redação final \\ da dissertação de mestrado devidamente \\ corrigida e defendida por \\ Marcelo Magalhães Taddeo \\ e aprovada pela comissão julgadora.
}

São Paulo, maio de 2003.

Banca examinadora:

- Prof. Dr. Pedro Paulo Schirmer - IME-USP

- Prof. Dr. Henrique Von Dreifus - IME-USP

- Prof. Dr. Rogério Rosenfeld - IFT-UNESP 

Dedicado a minha família. 


\section{Resumo}

Nosso objetivo é a determinação de uma medida de equilíbrio em mercados incompletos e a determinação da estrutura de volatilidade de ativos financeiros. Para isso nós introduzimos dentro da teoria financeira os principais conceitos da teoria da informação: entropia e entropia relativa. Nós mostramos como a entropia pode ser entendida como uma medida de incerteza e como essa interpretação é repassada para o ambiente de finanças. Mais ainda, fornecemos uma caracterização financeira para a entropia.

Tratamos também de problemas de otimização com restrições aplicada a entropia (maximização) e entropia relativa (minimização). Essas são questões importantes para a determinação da medida de Arrow-Debreu. A determinação dessa medida surge como consequência de dois princípios duais os quais denominamos de Princípio da Máxima Entropia e Princípio da Mínima Entropia Relativa. Mostramos também que esses princípios são compatíveis com a teoria corrente. De fato, a partir deles é possível determinar, de forma simplificada, a medida usada na teoria de apreçamento de derivativos de Black e Scholes.

Finalmente, expomos um modelo intertemporal para a determinação da estrutura de volatilidade de um ativo qualquer a partir de um prior. A estrutura de volatilidade surge como o resultado da minimização da entropia relativa impondo restrições sobre o valor descontado dos fluxos de caixa. 


\section{Agradecimentos}

A realização desse trabalho não se deve ao esforço de uma única pessoa. Gostaria de agradecer a todos aqueles que comigo colaboraram. Em particular, gostaria de agradecer a meu orientador e amigo Prof. Pedro Paulo Schirmer que, além de exercer com maestria o papel de orientador, expandiu minha percepção em relação ao que significa aplicar a matemática ao mundo real e em relação a beleza contida em finanças. Agradeço também aos meus pais, Mara e José Carlos, pelo exemplo, apoio e colaboração que eles têm me dado a vida inteira e aos quais estarei sempre em débito; a meu irmão, Guto, pela inestimável amizade; a minha namorada Patrícia (Pixy) por todos os bons momentos em que estivemos juntos, pela companhia e pela acessoria para assuntos gerais; aos amigos André, Cris, Márcio e Mariela; aos membros da banca examinadora Prof. Henrique von Dreifus e Prof. Rogério Rosenfeld; aos professores Antonio Luis Pereira, Edson de Faria e Iracema Bund; e, finalmente, à CNPq pelo auxílio financeiro que possibilitou a execução desse trabalho. 


\section{Sumário}

1 Teoria da Informação - Preliminares 1

1.1 Introdução - O Problema . . . . . . . . . . . . . . . . . . 1

1.2 Teoria da Informação - Conceitos Gerais . . . . . . . . . . . . 2

1.2.1 Entropia - Caso Discreto . . . . . . . . . . . . . . . 2

1.2.2 Propriedades da Entropia . . . . . . . . . . . . . . 4

1.2.3 Um Teorema de Existência e Unicidade . . . . . . . . . . 8

1.2.4 Entropia - Caso Contínuo ............. . . 11

1.2 .5 Entropia Relativa . . . . . . . . . . . . . . . . . . 19

1.2 .6 A Distribuição Normal . . . . . . . . . . . . . . . . . . 23

1.2.7 Propriedades da Entropia Relativa . . . . . . . . . . . . 28

1.2.8 A Desigualdade de Csiszár . . . . . . . . . . . . . . . 32

1.2.9 Representação Dual da Entropia Relativa . . . . . . . . . 39

1.2.10 Caracterização Financeira da Entropia . . . . . . . . . . 42

2 Teoria Entrópica de Apreçamento $\quad 45$

2.1 Introdução . . . . . . . . . . . . . . . . . . . . 45

2.2 Entropia em Mercados Incompletos . . . . . . . . . . . . . . . 46

2.3 Minimizando a Entropia Relativa . . . . . . . . . . . . . . 51

2.3.1 Casos Discreto e Contínuo . . . . . . . . . . . . . . 51

2.3.2 Caso Geral . . . . . . . . . . . . . . . . . 54

2.4 Maximizando a Entropia . . . . . . . . . . . . . . . 61

2.5 Aplicação do Princípio Entrópico . . . . . . . . . . . . . . . 63

2.6 Núcleo de Apreçamento e Entropia . . . . . . . . . . . . . . . . . 68

2.7 Entropia e o Modelo de Black-Scholes . . . . . . . . . . . . . 70

2.8 Entropia Relativa e a Informação de Fisher . . . . . . . . . . . 73

2.8.1 A Desigualdade de Cramér-Rao . . . . . . . . . . . . 73

2.8.2 Entropia Relativa e a Informação de Fisher . . . . . . . . 76

3 Modelos Intertemporais $\quad 79$

3.1 Calibrando uma Superfície de Volatilidade . . . . . . . . . . . . . 79

3.1.1 Aproximando a Entropia Relativa via Árvores Trinomiais 79

3.2 Problema de Controle Estocástico . . . . . . . . . . . . . 88 



\section{Capítulo 1}

\section{Teoria da Informação - Preliminares}

\subsection{Introdução - O Problema}

\section{Mercados Completos e Incompletos}

O Princípio da Não-Arbitragem, que afirma não existirem oportunidades de arbitragem no mercado, exerce um papel central na teoria de apreçamento de ativos, pois garante a existência de pelo menos uma medida de Arrow-Debreu. A última, por sua vez, permite o apreçamento de ativos. Basicamente, a medida de Arrow-Debreu é uma medida de probabilidade para a qual o retorno esperado descontado pela taxa livre de risco de qualquer ativo deve igualar o valor presente do ativo, ou seja, é como se ela fosse "cega" ou "indiferente" ao risco. O problema é que apesar de termos garantida a existência de tal medida não sabemos nada a respeito da unicidade e, portanto, classificamos os mercados relativamente a essa unicidade. Mais precisamente dividimos o mercado em duas categorias, as quais denominamos de mercado completo e mercado incompleto. Como veremos no próximo capítulo denominamos o mercado de completo se dado qualquer vetor de payoffs pré-determinado podemos atingi-lo com uma escolha correta do portfólio. ${ }^{1}$ Caso contrário, isto é, se existir um vetor de payoffs que não pode ser atingido por nenhum portfólio, então, dizemos que o mercado é incompleto. Ocorre que para mercados completos existe uma única medida de Arrow-Debreu, enquanto que para mercados incompletos não há unicidade. Todos esses conceitos serão expostos de modo mais preciso no próximo capítulo quando, enfim, aplicaremos as ferramentas matemáticas introduzidas nesse capítulo ao problema de apreçamento.

No caso em que o mercado é completo, o problema de apreçamento está bem-definido pois há exatamente uma medida de Arrow-Debreu. Portanto, o foco dessa dissertação reside, principalmente, no caso em que os mercados são

\footnotetext{
${ }^{1}$ Veja [6] para mais detalhes.
} 
incompletos sendo nosso objetivo propor um método para a determinação da "melhor" medida de Arrow-Debreu entre todas as possibilidades. Essa questão não é nova e diversos métodos já foram propostos, porém, muitos deles não demonstraram serem muito eficientes. Os dois principais critérios consideram a distribuição de mínima variância ou a estrutura de preferência do investidor através da maximização da função de utilidade (nesse caso, retornando ao antigo inconveniente da necessidade de se levantar hipóteses acerca do comportamento dos investidores, ou seja, da função de utilidade, o qual havia sido eliminado no caso em que os mercados são completos pela teoria de apreçamento de ativos desenvolvida por Harrison, Kreps e Pliska). O método que estudamos aqui utiliza a teoria da informação de Shannon para determinar qual medida de Arrow-Debreu escolher. Basicamente, ele procura determinar a distribuição ou medida de probabilidade que maximiza a entropia. A entropia é o principal ente da teoria da informação e serve como uma medida do grau de desordem embutido numa dada medida de probabilidade ou, se preferirmos, como um quantificador da informação contida na mesma. Em outros casos, o método procura a medida de probabilidade que minimiza a entropia relativa entre uma distribuição ou medida de probabilidade dada a priori (também conhecida por prior ou medida de referência). A entropia relativa é um outro elemento da teoria da informação de extrema importância e que em um certo sentido faz o papel de uma métrica no espaço das medidas de probabilidade. Como veremos mais adiante esse é um método que não depende dos agentes e que, portanto, independe da estrutura de preferência dos mesmos mas que, sendo em certas ocasiões um método bayesiano, incorpora as convicções dos agentes no momento da escolha do prior. Nas próximas seções apresentaremos as principais definições e resultados, as quais sozinhas justificam seu uso em finanças e as encerraremos fornecendo uma caracterização monetária da entropia relativa.

\subsection{Teoria da Informação - Conceitos Gerais}

\subsubsection{Entropia - Caso Discreto}

O conceito o qual focaremos é a entropia. Comecemos definindo o caso discreto e enunciando em seguida algumas de suas propriedades e exemplos os quais ilustrem seu significado como uma medida de incerteza. Seja $X$ uma variável aleatória, definida em um espaço $\Omega$ dado, e suponhamos que $X$ assuma os valores $x_{1}, \ldots, x_{m}$. A variável aleatória $X$ está associada uma incerteza com respeito a suas realizações, a qual é avaliada através de uma medida de probabilidade $\mathbf{P}$ que a cada possível resultado associa um peso denotados da seguinte forma:

$$
\mathbf{P}\left(X=x_{i}\right):=p_{i}
$$

para $i=1, \ldots, m$. A pergunta que nos fazemos é quanta informação tal distribuição de probabilidades carrega a respeito das realizações futuras de tal variável aleatória (ou qualquer outra cuja distribuição seja idêntica a acima)? Para respondê-la definimos o conceito de entropia. 
Definição 1 Seguindo a notação acima, definimos a entropia de $X$ (ou de $\mathbf{p}:=$ $\left.\left(p_{1}, \ldots, p_{m}\right)\right)$ por:

$$
h(X) \equiv h(\mathrm{p}):=-\sum_{j=1}^{m} p_{j} \log p_{j}
$$

Note que introduzimos duas notações para uma mesma grandeza, a dizer, $h(X)$ e $h(\mathbf{p})$, as quais usaremos sem medo de confusão. No entanto, preferimos utilizar a segunda delas, pois entendemos que a entropia mede o grau de incerteza intrínseco da distribuição de probabilidade e, portanto, independe da variável aleatória definida em $\Omega$. Além disso, não especificamos a base do logaritmo, pois seu valor não é relevante para o desenvolvimento da teoria uma vez que as propriedades da função logaritmo independem da mesma. Ainda assim, costuma-se trabalhar na base 2 , sendo a entropia expressa em bits, ou na base $e$, expressando a entropia em nats. A razão da primeira escolha provém da teoria da comunicação, enquanto que a segunda costuma ser utilizada devido às suas vantagens computacionais.

Também, a partir de agora adotaremos a seguinte convenção:

$$
0 \log 0=0 .
$$

$\mathrm{Na}$ verdade $\log 0$ não está definido, mas, $\lim _{x \rightarrow 0} x \log x=0 \mathrm{e}$, portanto, essa convenção está baseada nesse limite. Nós a adoteremos para simplificar a argumentação que segue abaixo. Um outro modo de escrever a definição de entropia e que não envolve nenhuma convenção é $h(\mathrm{p})=-\sum_{j=1}^{m} \phi\left(p_{j}\right)$, onde $\phi\left(p_{j}\right)=p_{j} \log p_{j}$ se $p_{j}>0$ e $\phi\left(p_{j}\right)=0$, caso contrário. No entanto, consideramos o primeiro modo mais intuitivo e, consequentemente, o manteremos.

A seguir calculamos explicitamente a entropia em dois casos distintos a título de ilustração:

\section{Exemplo 1 (Distribuição Uniforme)}

Seja $\Omega=\left(\omega_{1}, \ldots, \omega_{N}\right)$ com $N \in \mathbb{N}$ e suponha que sobre esse conjunto tenhamos uma distribuição uniforme, ie, $\mathbf{p}_{N}=\left(\frac{1}{N}, \ldots, \frac{1}{N}\right)$. Vamos calcular a entropia dessa distribuição:

$$
h\left(\mathbf{p}_{N}\right):=-\sum_{j=1}^{N} p_{j} \log p_{j}=-\sum_{j=1}^{N} \frac{1}{N} \log \frac{1}{N}=\log N
$$

Observe que a entropia é uma função crescente de $N$ e que $\lim _{N \rightarrow \infty} \mathbf{p}_{N}=\infty$. Isso está de acordo com a nossa expectativa, pois, numa distribuição uniforme, quanto maior for o número de estados da natureza, maior é a incerteza com relação ao resultado do experimento, pois qualquer resultado tem a mesma probabilidade de ocorrência. 


\section{Exemplo 2 (Experimento de Bernoulli)}

Considere um experimento de Bernoulli, com probabilidade de sucesso $p$. Neste caso a entropia é dada por:

$$
h((p, 1-p))=p \log p+(1-p) \log (1-p)
$$

Note que para $p=0$ ou $p=1$, a entropia se anula, pois, neste caso não nenhuma incerteza quanto ao resultado, ou seja, não há aleatoriedade no experimento. Além disso, ela atinge seu máximo em $p=\frac{1}{2}$, novamente exprimindo caráter de uma medida de incerteza, dado que com $p=\frac{1}{2}$ é quase impossível fazer alguma previsão de qual realização realmente ocorrerá, o que caracteriza um alto grau de incerteza.

Apesar de assumirmos que a variável aleatória $X$ assume apenas um número finito de valores, isso não é realmente necessário e a generalização para o caso em que assume-se uma quantidade infinita, porém enumerável, de valores (ou, equivalentemente, no caso em que as medidas de probabilidade moram no espaço das sequências cujos elementos são não-negativos e cuja soma dos mesmos é igual a um) é imediata. De fato se $X$ assume os valores $x_{1}, x_{2}, \ldots$ com probabilidade $\mathbf{p}=\left(p_{1}, p_{2}, \ldots\right)$, então:

$$
h(X)=h(\mathbf{p})=-\sum_{i=1}^{\infty} p_{i} \log p_{i}
$$

Note que se a sequência $\left(\log p_{i}\right)_{i} \in l^{1}(\mathbb{N})$, então, a sequência acima é convergente.

\subsubsection{Propriedades da Entropia}

Nesta seção provaremos algumas propriedades da entropia no caso discreto e esperamos com isso tentar convencer o leitor da vedadeira natureza desse conceito. No entanto, começaremos demonstrando um lema que nos será útil a seguir em diversas outras ocasiões:

Lema 1 A função:

$$
\phi(x)= \begin{cases}x \log x & \text { se } x>0, \\ 0 & \text { caso contrário. }\end{cases}
$$

é convexa.

Prova. Analisemos essa função restrita ao intervalo $(0, \infty)$. Temos $\phi^{\prime}(x)=$ $1+\log x$ e, portanto, estritamente crescente. Logo, $\phi$ é estritamente convexa em $(0, \infty)$. Agora seja $\epsilon>0$, então,

$$
\frac{\phi(\epsilon)-\phi(0)}{\epsilon}=\log \epsilon<0
$$


para $\epsilon$ suficientemente pequeno. Portanto, a função $\phi$ é decrescente em uma vizinhança de 0 . Consequentemente, $\phi$ é estritamente convexa em $[0, \infty)$.

Seguindo a mesma notação da seção anterior:

Teorema 1 As seguintes afirmações são verdadeiras:

1. A entropia é sempre não-negativa: $h(X) \geq 0$;

2. A entropia anula-se se, e somente se, $p_{j}=1$ para algum $j=1,2, \ldots$ (e, consequentemente, igual a zero nos demais $j$ 's);

3. Se a variável aleatória $X$ assume um número finito de valores, digamos $n$ valores, então sua entropia atinge o máximo exatamente quando os $p_{j}$ 's são todos idênticos, isto é, $p_{j}=\frac{1}{n}$ para todo $j=1, \ldots, n$. Em particular, $h(X) \leq \log n$.

Prova.O primeiro item do teorema segue do fato que $0 \leq p_{i} \leq 1$ e, portanto, $\log p_{i} \leq 0, \log \mathrm{o},-\sum_{i} p_{i} \log p_{i} \geq 0$.

Agora, se existe $m$ tal que $p_{m}=1$, então todos os $p_{j}$ 's restantes anulam-se e, consequentemente, pela nossa convenção $p_{j} \log p_{j}=0$. E como $p_{m} \log p_{m}=$ $1 \log 1=0$ segue que $h(X)=0$. Por outro lado, se $h(X)=0$ então $p_{j} \log p_{j}=0$ para todo $j$ e, portanto, dado $j$ tem-se $p_{j} \log p_{j}=0$ ou $p_{j} \log p_{j}=1, \log , p_{j}=0$ ou $p_{j}=1$. Consequentemente, segue das propriedades de uma distribuição de probabilidade que existe um único $j$ tal que $p_{j}=1$ de modo que as demais componentes dessa distribuição anulam-se.

Resta mostrarmos a terceira parte do teorema. Consideremos a função $\phi(x):=x \log x$, a qual sabemos é convexa e, portanto, tem-se $\phi\left(\frac{1}{n} \sum_{j=1}^{n} x_{j}\right) \leq$ $\frac{1}{n} \sum_{j=1}^{n} \phi\left(x_{j}\right)=\frac{1}{n} \sum_{j=1}^{n} x_{j} \log x_{j}$. Substituindo $x_{j}$ por $p_{j}$ e lembrando que $\sum_{j=1}^{n} p_{j}=1$ tem-se que $\phi\left(\frac{1}{n}\right) \leq-\frac{1}{n} h\left(p_{1}, \ldots, p_{n}\right), \operatorname{logo}, h(X) \leq-n\left(\frac{1}{n} \log \frac{1}{n}\right)=$ $h\left(\frac{1}{n}, \ldots, \frac{1}{n}\right)$, qualquer que seja a distribuição de probabilidade $\left(p_{1}, \ldots, p_{n}\right)$ associada a $X$, e assim demonstramos a primeira afirmação do terceiro item do teorema. A segunda afirmação desse item segue do cálculo explicito da entropia da distribuição uniforme, o qual resulta em: $h\left(\frac{1}{n}, \ldots, \frac{1}{n}\right)=\log n$.

Então, quando um evento é certo, a entropia é mínima, ou seja, não há mais nenhuma quantidade de informação disponível. Conhecemos o resultado sem nenhuma dúvida. Por outro lado, quando todos eventos têm a mesma probabilidade de ocorrer, então não é possível extrair nenhuma informação de sua distribuição de probabilidade no sentido de obtermos alguma previsão, isto é, a entropia é máxima.

A entropia pretende medir a quantidade de informação que uma certa distribuição de probabilidade transmite em relação às possíveis realizações de uma 
variável aleatória. Pode-se imaginar essa variável aleatória como um experimento e, agora, imaginemos que um outro experimento é realizado concomitantemente àquele representado por $X$. Esse novo experimento pode nos dar informação extra com relação ao primeiro, isto é, com relação a $X$. Para medirmos esse acréscimo informacional introduzimos o conceito de entropia condicional.

Representemos o segundo experimento por uma variável aleatória $Y$ a qual assume os valores $\left(y_{1}, \ldots, y_{n}\right)$ e definamos $\mathbf{P}\left(Y=y_{j}\right):=q_{j}$ e $\mathbf{P}\left(X=x_{i}, Y=\right.$ $\left.y_{j}\right):=r_{i j}$, para todos $i=1, \ldots, m$ e $j=1, \ldots, n$. Se $\mathbf{P}\left(X=x_{i} \mid Y=y_{j}\right):=p_{i \mid j}$, então, $r_{i j}=p_{i \mid j} q_{j}$ e observemos, também, que se $X$ e $Y$ são independentes, então, $r_{i j}=p_{i} q_{j}$, para todos $i=1, \ldots, m$ e $j=1, \ldots, n$. Assumindo essas notações enunciemos a seguinte definição:

Definição $2 A$ entropia condicional de $X$ dado que $Y=y_{j}$ é dada por $h\left(X \mid Y=y_{j}\right):=-\sum_{i=1}^{m} p_{i \mid j} \log p_{i \mid j} . A$ entropia condicional de $X$ dado $Y$, $h(X \mid Y)$, é o valor esperado das entropias condicionais definidas acima, ou seja,

$$
h(X \mid Y)=\sum_{j=1}^{n} q_{j} h\left(X \mid Y=y_{j}\right) .
$$

Para cada $j$ fixado o vetor $\left(p_{1 \mid j}, \ldots, p_{m \mid j}\right)$ define uma nova distribuição de probabilidade sobre os resultados de $X$, portanto, $h\left(X \mid Y=y_{j}\right)$ é a entropia da variável aleatória sob a nova distribuição. Ou seja, quando tomamos conhecimento do resultado de um novo experimento a incerteza com relação aos resultados do experimento original alteram-se. A intuição nos diz que ela deve diminuir, ou no máximo manter-se inalterada (caso ambos sejam independentes), pois nova informação é fornecida. É exatamente isso que afirma o próximo resultado:

Teorema 2

$$
h(X \mid Y) \leq h(X) .
$$

$E$ se $X$ e $Y$ são independentes, então vale a igualdade.

Prova. Novamente estaremos usando a convexidade da função $\phi(x)=x \log x$. De fato,

$$
\begin{aligned}
h(X \mid Y) & =\sum_{j=1}^{n} q_{j} h\left(X \mid Y=y_{j}\right)=-\sum_{j=1}^{n} q_{j} \sum_{i=1}^{m} p_{i \mid j} \log p_{i \mid j} \\
& =-\sum_{i=1}^{m}\left(\sum_{j=1}^{n} q_{j} \phi\left(p_{i \mid j}\right)\right)
\end{aligned}
$$


Como $\phi$ é convexa, segue que $\phi\left(\sum_{j=1}^{n} q_{j} x\right) \leq \sum_{j=1}^{n} q_{j} \phi(x)$, logo,

$$
\begin{aligned}
h(X \mid Y) & \leq-\sum_{i=1}^{m}\left(\sum_{j=1}^{n} q_{j} p_{i \mid j}\right) \log \left(\sum_{j=1}^{n} q_{j} p_{i \mid j}\right) \\
& =-\sum_{i=1}^{m} p_{i} \log p_{i}=h(X) .
\end{aligned}
$$

Assumindo a independência entre $X$ e $Y$ temos:

$$
p_{i \mid j}=\frac{r_{i, j}}{q_{j}}=\frac{p_{i} q_{j}}{q_{j}}=p_{i}
$$

logo, $h\left(X \mid Y=y_{j}\right)=-\sum_{i=1}^{m} p_{i} \log p_{i}=h(X)$. E, portanto,

$$
h(X \mid Y)=\sum_{j=1}^{n} q_{j} h\left(X \mid Y=y_{j}\right)=h(X) \sum_{j=1}^{n} q_{j}=h(X)
$$

ou seja, vale a igualdade em (1.5)

Em outras palavras, o conhecimento de uma distribuição (associado a algum experimento) nunca diminui a quantidade de informação obtida de uma outra distribuição, podendo, eventualmente, até mesmo aumentá-la. Caso duas distribuições sejam independentes, então uma não contribui em nada à outra no sentido de diminuir suas respectivas entropias.

Teorema 3 Usando a mesma notação acima, tem-se:

$$
h(X, Y):=h(\mathbf{r})=h(Y)+h(X \mid Y) .
$$

Prova

$$
\begin{aligned}
h(X, Y) & =-\sum_{i=1}^{m} \sum_{j=1}^{n} r_{i j} \log r_{i j}=-\sum_{i=1}^{m} \sum_{j=1}^{n} p_{i \mid j} q_{j} \log p_{i \mid j} q_{j} \\
& =-\sum_{i=1}^{m} \sum_{j=1}^{n} p_{i \mid j} q_{j} \log q_{j}-\sum_{i=1}^{m} \sum_{j=1}^{n} p_{i \mid j} q_{j} \log p_{i \mid j} \\
& =-\sum_{j=1}^{n} q_{j} \log q_{j} \sum_{i=1}^{m} p_{i \mid j}+\sum_{j=1}^{n} q_{j} h\left(X \mid Y=y_{j}\right) \\
& =h(Y)+h(X \mid Y)
\end{aligned}
$$

Corolário 1 Se $X$ e $Y$ são independentes, então:

$$
h(X, Y)=h(X)+h(Y)
$$

Prova. Esse corolário segue imediatamente da aplicação dos teoremas (2) e (3). 


\subsubsection{Um Teorema de Existência e Unicidade}

Nesta seção tentaremos justificar porque a entropia, no caso discreto, é definida da forma como foi acima. Ou seja, assumiremos algumas propriedades básicas que uma função que se proponha a medir a incerteza de distribuições de probabilidade ou variáveis aleatórias devem satisfazer, e a partir destas deduziremos a expressão dada na definição de entropia.

Algumas dessas propriedades foram enunciadas e demonstradas na seção anterior. Destaquemos duas:

Propriedade 1. Dado $n \in \mathbb{N}$, a função $h\left(p_{1}, \ldots, p_{n}\right)$ atinge seu máximo em

$\left(\frac{1}{n}, \ldots, \frac{1}{n}\right) ;$
$\mathrm{n}$ vezes

Propriedade 2. Usando a mesma notação da seção anterior, $h(X, Y)=$ $h(Y)+h(X \mid Y)$, onde $h(X \mid Y)=\sum_{j=1}^{n} q_{j} h\left(X \mid Y=y_{j}\right)$ e se $X$ e $Y$ são independentes, então, $h(X \mid Y)=h(X)$.

Os significados de ambas as propriedades já foram discutidas na seção anterior. A elas, adicionemos mais uma:

Propriedade 3. Seja $\mathbf{p}=\left(p_{1}, \ldots, p_{n}\right)$ uma distribuição de probabilidade qualquer. Então, $h\left(p_{1}, \ldots, p_{n}\right)=h\left(p_{1}, \ldots, p_{n}, 0\right)$.

Ou seja, a inclusão de eventos impossíveis não altera a entropia de uma distribuição.

As propriedades (1), (2) e (3) são bastante naturais e é razoável assumir que qualquer medida ou informacional ou de incerteza as satisfaçam. O resultado a seguir nos diz que a definição que demos acima para a entropia pode, na verdade, ser deduzida se assumimos as propriedades acima como axiomas (axiomas de informação):

Teorema 4 Se $h$ é uma função contínua não-constante definida no espaço das distribuições de probabilidade para um número finito de estados (isto é, no espaço dos elementos $\left(p_{1}, \ldots, p_{n}\right)$ tal que $p_{j} \geq 0$, para todo $j=1, \ldots, n$ e $\sum_{j=1}^{n} p_{j}=1$ para todo $n \in \mathbb{N}$ ) satisfazendo as propriedades 1 , 2 e 3 acima, então,

$$
h\left(p_{1}, \ldots, p_{n}\right)=-\lambda \sum_{j=1}^{n} p_{j} \log p_{j},
$$

onde $\lambda$ é alguma constante positiva.

Prova. Comecemos demonstrando um caso particular do teorema o qual usaremos na demonstração do caso geral. Para todo inteiro positivo $n$ definamos:

$$
f(n):=h\left(\frac{1}{n}, \ldots, \frac{1}{n}\right)
$$


de modo que queremos mostrar a igualdade:

$$
f(n)=\lambda \log n,
$$

para alguma constante positiva $\lambda$.

Das propriedades 1 e 3 acima, temos:

$$
f(n)=h\left(\frac{1}{n}, \ldots, \frac{1}{n}\right)=h\left(\frac{1}{n}, \ldots, \frac{1}{n}, 0\right) \leq h\left(\frac{1}{n+1}, \ldots, \frac{1}{n+1}\right)=f(n+1)
$$

$\operatorname{logo}, f$ é uma função não-decrescente de $n$. Consideremos agora as $m$ seguintes variáveis aleatórias $X_{1}, \ldots, X_{m}$, cada uma das quais assumindo $k$ valores distintos igualmente prováveis, ou seja, assumimos que cada uma dessas variáveis aleatória tem distribuição uniforme $U(k)$. Então,

$$
h\left(X_{j}\right)=h\left(\frac{1}{k}, \ldots, \frac{1}{k}\right)=f(k)
$$

para $j=1, \ldots, m$. Pela segunda propriedade de $h$, temos:

$$
h\left(X_{1}, \ldots, X_{m}\right)=\sum_{j=1}^{m} h\left(X_{j}\right)=m f(k) .
$$

Por outro lado, a variável aleatória $Z:=\left(X_{1}, \ldots, X_{m}\right)$ assume $k^{m}$ valores igualmente prováveis e, portanto, $h(Z)=f\left(k^{m}\right)$. Consequentemente,

$$
f\left(k^{m}\right)=m f(k) .
$$

Como $m$ e $k$ foram escolhidos arbitrariamente, segue que dado $l$, um inteiro positivo, também vale que:

$$
f\left(l^{n}\right)=n f(l) .
$$

Agora, suponhamos que $k, l$ e $n$ são dados (escolhidos arbitrariamente) e tomemos $m$ tal que:

$$
k^{m} \leq l^{n} \leq k^{m+1},
$$

então, como a função log é monótona, segue que:

$$
m \log k \leq n \log l \leq(m+1) \log k,
$$

ou seja,

$$
\frac{m}{n} \leq \frac{\log l}{\log k} \leq \frac{m}{n}+\frac{1}{n}
$$

Por outro lado, da monotonicidade da função $f$ :

$$
f\left(k^{m}\right) \leq f\left(l^{n}\right) \leq f\left(k^{m+1}\right),
$$

e, consequentemente,

$$
m f(k) \leq n f(l) \leq(m+1) f(k),
$$


$\log$,

$$
\frac{m}{n} \leq \frac{f(l)}{f(k)} \leq \frac{m}{n}+\frac{1}{n}
$$

Segue, portanto, das equações (1.11) e (1.12):

$$
\left|\frac{f(l)}{f(k)}-\frac{\log l}{\log k}\right| \leq \frac{1}{n}
$$

e como o lado esquerdo da desigualdade acima independe de $n$ temos:

$$
\frac{f(l)}{\log l}=\frac{f(k)}{\log k} .
$$

Então, devido a arbitrariedade de $l$ e $k$, deve existir uma constante $\lambda$ tal que:

$$
h\left(\frac{1}{n}, \ldots, \frac{1}{n}\right)=f(n)=\lambda \log n,
$$

e, como $f$ é não-decrescente, $\lambda$ é não-negativa. Isso mostra a afirmação feita no início da demonstração.

Resumindo, mostramos o teorema no caso particular em que $p_{j}=\frac{1}{n}$ para $j=1, \ldots, n$. Vamos agora analisar o caso mais geral em que $p_{j} \in \mathbb{Q}$. Nesse caso, podemos escrever $p_{j}=\frac{\xi_{j}}{\xi}$, onde $\xi_{j}$ e $\xi$ são números inteiros não negativos, $\xi \neq 0$, e $\sum_{j=1}^{n} \xi_{j}=\xi$. Consideremos então a variável aleatória $Y$ assumindo $n$ valores $y_{1}, \ldots, y_{n}$ com probabilidades $p_{1}, \ldots, p_{n}$. Nosso objetivo é determinar a expressão de $h(Y)$ dadas as propriedades enunciadas mais acima. Para isso, definamos uma outra variável aleatória $X$ dependente de $Y$ da seguinte maneira: $X$ assume valores $x_{1}, \ldots, x_{\xi}$ os quais dividimos em $n$ grupos disjuntos $\chi_{1}, \ldots, \chi_{n}$ contendo $\xi_{1}, \ldots, \xi_{n}$ elementos de $\left\{x_{1}, \ldots, x_{\xi}\right\}$, respectivamente, cuja reunião preenche esse conjunto. Assumiremos também que se conhecemos qual valor foi assumido por $Y$, digamos $Y=y_{k}$, para algum $k=1, \ldots, n$, então $\mathbf{P}(X=x)=\frac{1}{\xi_{k}}$, se $x \in \chi_{k}$, e $\mathbf{P}(X=x)=0$, caso contrário. Ou seja, dada a ocorrência de um elemento $y_{k}$, então, as possíveis realizações da variável aleatória $X$ reduz-se aos elementos de $\chi_{k}$ com probabilidades de ocorrência iguais. Consequentemente, temos:

$$
h\left(X \mid Y=y_{k}\right)=h\left(\frac{1}{\xi_{k}}, \ldots, \frac{1}{\xi_{k}}\right)=f\left(\xi_{k}\right)=\lambda \log \chi_{k}
$$

conforme a primeira parte dessa demonstração. Logo,

$$
\begin{aligned}
h(X \mid Y) & =\sum_{j=1}^{n} p_{j} h\left(X \mid Y=y_{j}\right)=\lambda \sum_{j=1}^{n} p_{j} \log \xi_{j} \\
& =\lambda \sum_{j=1}^{n} p_{j} \log p_{j} \xi
\end{aligned}
$$

e, portanto

$$
h(X \mid Y)=\lambda \sum_{j=1}^{n} p_{j} \log p_{j}+\lambda \sum_{j=1}^{n} p_{j} \log \xi
$$


Agora consideremos a variável aleatória $Z=(X, Y)$. Uma realização de, digamos $Z=\left(x_{k}, y_{l}\right)$, é possível se, e somente se, $x_{k} \in \chi_{l}$. Então, o número de possíveis realizações $\left(x_{k}, y_{l}\right)$ dado $l$ é $\xi_{l}$, logo, a quantidade total delas é $\sum_{j=1}^{n} \xi_{j}=\xi$. Além disso,

$$
\mathbf{P}\left(Z=\left(x_{k}, y_{l}\right)\right)=\mathbf{P}\left(X=x_{k} \mid Y=y_{l}\right) \mathbf{P}\left(Y=y_{l}\right)=\frac{p_{l}}{\xi_{l}}=\frac{1}{\xi},
$$

ou seja, todas realizações de $Z$ têm a mesma probabilidade de ocorrência, de modo que:

$$
h(X, Y)=h(Z)=\lambda \log \chi .
$$

Pela segunda propriedade e pelas equações (1.15) e (1.16):

$$
\lambda \log \xi=h(X, Y)=h(Y)+h(X \mid Y)=h(Y)+\lambda \sum_{j=1}^{n} p_{j} \log p_{j}+\lambda \log \xi,
$$

portanto,

$$
h(Y)=-\lambda \sum_{j=1}^{n} p_{j} \log p_{j} .
$$

Demonstramos assim o teorema no caso em que $p_{j}$ é racional para todo $j=1, \ldots, n$, com $n$ inteiro positivo e arbitrário. Como estamos assumindo $h$ contínua, então, o resultado deve valer para todo $p_{j} \in \mathbb{R}$, satisfazendo $p_{j} \geq 0 \mathrm{e}$ $\sum p_{j}=1$. Além disso, $h$ não-constante implica $\lambda$ estritamente positivo.

A magnitude de $\lambda$ não importa muito, pois isso significa apenas uma mudança de unidades, logo, sem perda de generalidade, podemos tomar $\lambda=1$.

\subsubsection{Entropia - Caso Contínuo}

Nessa seção introduziremos o conceito de entropia no caso em que as variáveis aleatórias (ou suas distribuições de probabilidade associadas) são contínuas. Quando houver a necessidade de distinção, denominaremos a entropia, no caso em que a variável aleatória é contínua, por entropia contínua (é comum encontrar na literatura também a denominação entropia diferencial), e no caso em que a variável aleatória é discreta por entropia discreta. Veremos que ambas coincidem em muitos pontos, mas não em todos e que, além disso, uma não é o caso limite da outra. Como na seção anterior, seja $X$ uma variável aleatória assumindo valores em $\mathbb{R}^{n}$ e seja $f=f\left(x_{1}, \ldots, x_{n}\right)$ a função densidade de probabilidade associada a $X$. Então:

Definição $3 A$ entropia de $X$ (ou de $f$ ) é dada por:

$$
h(X)=h(f):=-\int_{\mathbb{R}} \log (f(x)) f(x) d x
$$

caso a integral acima exista. 
O próximo exemplo já ilustra o primeiro contraste com o caso discreto, a dizer, a entropia contínua pode assumir valores negativos:

\section{Exemplo 3 (Distribuição Uniforme)}

Suponha $n=1$ e que $X$ tenha uma distribuição uniforme, digamos,

$$
f(x)= \begin{cases}\frac{1}{b-a} & \text { se } x \in(a, b) \\ 0 & \text { caso contrário }\end{cases}
$$

onde $b>a$ são números arbitrários (a escolha do intervalo $(a, b)$ aberto foi arbitrária, podendo ser esse semi-aberto ou mesmo fechado sem que isso prejudicasse o argumento). Então,

$$
h(X)=-\int_{a}^{b} \log \left(\frac{1}{b-a}\right) \frac{1}{b-a} d x=\log (b-a) .
$$

Agora, se $b-a<1$, então, $h(X)<0$ ! Além disso, quanto maior for o intervalo $(a, b)$, maior será a entropia, ou em termos informacionais, quanto maior o intervalo $(a, b)$, menos informação nos é dada com relação aos possíveis eventos.

Uma outra característica da entropia contínua é que essa não é simplesmente o caso discreto visto no limite. De fato, seja $X$ uma variável aleatória com função densidade de probabilidade $f(x)$ e, então, definamos as seguintes variáveis aleatórias: fixado $n \in \mathbb{N}$ qualquer, tomemos:

$$
X_{n}:=\frac{k}{n} \text { se } \frac{k}{2^{n}} \leq X<\frac{k+1}{2^{n}}
$$

onde $k \in \mathbb{Z}$. É evidente que para todo $n$, as $X_{n}^{\prime} s$ são variáveis aleatórias discretas e estão definidas no mesmo espaço que $X$. Segue também da definição dessas variáveis que $X_{n} \rightarrow X$ quando $n \rightarrow \infty$ na norma $L^{p}(f(x) d x)$, para qualquer $1 \leq p \leq \infty$. Para cada $n$ e cada $k$ fixados, definamos os intervalos semi-abertos:

$$
I_{n, k}:=\left[\frac{k}{2^{n}}, \frac{k+1}{2^{n}}\right)
$$

e, então, para cada $n$ podemos atribuir uma distribuição de probabilidade para a variável $X_{n}$ dada por:

$$
\mathbf{P}\left(X_{n}=\frac{k}{2^{n}}\right)=\mathbf{P}\left(X \in I_{n, k}\right)=\int_{I_{n, k}} f(x) d x .
$$

Por questão de simplicidade definamos

$$
\mathbf{P}\left(X_{n}=\frac{k}{2^{n}}\right):=\varphi_{n, k}
$$


para todos $n \in \mathbb{N}$ e $k \in \mathbb{Z}$. Resumindo, acabamos de construir uma sequência de variáveis aleatórias $\left(X_{n}\right)_{n \in \mathbb{N}}$, que converge para $X$, dotadas, para cada $n$, de uma distribuição de probabilidade $\left(\ldots, \varphi_{n,-1}, \varphi_{n, 0}, \varphi_{n, 1}, \ldots\right)$. Consequentemente, a entropia de $X_{n}$ é:

$$
h\left(X_{n}\right)=-\sum_{k=-\infty}^{\infty} \varphi_{n, k} \log \varphi_{n, k} .
$$

No entanto, a soma acima diverge quando $\varphi_{n, k} \rightarrow 0$, e é exatamente isso o que ocorre quando $X_{n} \rightarrow X$, ou seja, quando $n \rightarrow \infty, X_{n} \rightarrow X$, porém, $h\left(X_{n}\right) \nrightarrow h(X)$ !

Ora, mostramos então que não é necessariamente verdade que a entropia contínua de uma variável aleatória $X$ é o limite das entropias de variáveis aleatórias discretas que convergem para a mesma. A pergunta natural que surge é se existe alguma relação entre ambas, e se existe, qual sua representação. O próximo resultado esclarece essa questão:

Teorema 5 Seja $f$ uma função satisfazendo

1. f é limitada e contínua quase sempre;

2. $\int_{\mathbb{R}}|f(x) \log f(x)| d x<\infty$;

3. $h\left(X_{0}\right)=-\sum_{k=-\infty}^{\infty} \varphi_{0, k} \log \varphi_{0, k}<\infty$;

então,

$$
\lim _{n \rightarrow \infty}\left[h\left(X_{n}\right)-n \log 2\right]=h(X) .
$$

Prova. Definamos $f_{n}(x):=2^{n} \varphi_{n, k}$ se $x \in I_{n, k}$ para todos $n \in \mathbb{N}$ e $k \in \mathbb{Z}$. Então,

$$
\begin{aligned}
\int_{I_{n, k}} f_{n}(x) \log f_{n}(x) d x & =2^{n} \varphi_{n, k} \log \left(2^{n} \varphi_{n, k}\right)\left|I_{n, k}\right|=2^{n} \varphi_{n, k} \log \left(2^{n} \varphi_{n, k}\right) \frac{1}{2^{n}} \\
& =\varphi_{n, k} \log \left(2^{n} \varphi_{n, k}\right) .
\end{aligned}
$$

Então, como $\sum_{k} \varphi_{n, k}=1$ :

$$
\begin{aligned}
h\left(X_{n}\right)-n \log 2 & =-\sum_{k} \varphi_{n, k} \log \varphi_{n, k}-\log 2^{n} \sum_{k} \varphi_{n, k} \\
& =-\sum_{k} \varphi_{n, k} \log \left(2^{n} \varphi_{n, k}\right) \\
& =-\sum_{k} \int_{I_{n, k}} f_{n}(x) \log f_{n}(x) \\
& =-\int_{\mathbb{R}} f_{n} \log f_{n}(x) d x \\
& =-\int_{-K}^{K} f_{n}(x) \log f_{n}(x) d x-\int_{|x| \geq K} f_{n}(x) \log f_{n}(x) d x
\end{aligned}
$$


onde $K \in \mathbb{Z}_{+}$é arbitrário. Agora fixando $x_{0}, \lim _{n} f_{n}\left(x_{0}\right)=\lim _{n} 2^{n} \varphi_{n, k(n)}$, onde $k(n)$ é tal que $x_{0} \in I_{n, k(n)}$. Então,

$$
\lim _{n \rightarrow \infty} f_{n}\left(x_{0}\right)=\lim _{n \rightarrow \infty} \frac{1}{\left|I_{n, k(n)}\right|} \int_{I_{n, k(n)}} f(x) d x=f\left(x_{0}\right)
$$

pois, $f$ é contínua, logo, pelo teorema da convergência limitada:

$$
-\lim _{n \rightarrow \infty} \int_{-K}^{K} f_{n}(x) \log f_{n}(x) d x=-\int_{-K}^{K} f(x) \log f(x) d x
$$

e, pela propriedade (2):

$$
-\lim _{K \rightarrow \infty} \int_{|x| \geq K} f(x) \log f(x) d x=0 .
$$

Consequentemente, resta apenas mostrar que:

$$
\lim _{K \rightarrow \infty} \int_{|x| \geq K} f_{n}(x) \log f_{n}(x) d x=0
$$

uniformemente em $n$. De fato, neste caso teremos:

$$
\begin{aligned}
& \lim _{n \rightarrow \infty}\left(h\left(X_{n}\right)-n \log 2\right)=\lim _{K \rightarrow \infty} \lim _{n \rightarrow \infty}\left(h\left(X_{n}\right)-n \log 2\right)= \\
= & \lim _{K \rightarrow \infty} \lim _{n \rightarrow \infty}\left(-\int_{-K}^{K} f_{n}(x) \log f_{n}(x) d x-\int_{|x| \geq K} f_{n}(x) \log f_{n}(x) d x\right) \\
= & \lim _{K \rightarrow \infty}-\int_{-K}^{K} f(x) \log f(x) d x-\lim _{n \rightarrow \infty} \lim _{K \rightarrow \infty} \int_{|x| \geq K} f_{n}(x) \log f_{n}(x) d x \\
= & -\int_{\mathbb{R}} f(x) \log f(x) d x=h(X)
\end{aligned}
$$

que é exatamente a tese do teorema. Provemos, então, a igualdade (1.22).

Como

$$
\sum_{l 2^{n} \leq k<(l+1) 2^{n}} \varphi_{n, k}=\mathbf{P}(l \leq X<l+1)=\varphi_{0, l}
$$


e lembrando que $\phi(x)=x \log x$ é convexa, segue que:

$$
\begin{aligned}
-\int_{l}^{l+1} f_{n}(x) \log f_{n}(x) d x & =-\sum_{l 2^{n} \leq k<(l+1) 2^{n}} \int_{I_{n, k}} f_{n}(x) \log f_{n}(x) d x \\
& =\frac{1}{2^{n}}\left(-\sum_{l 2^{n} \leq k<(l+1) 2^{n}} 2^{n} \varphi_{n, k} \log \left(2^{n} \varphi_{n, k}\right)\right) \\
& =\frac{1}{2^{n}} \sum_{l 2^{n} \leq k<(l+1) 2^{n}}\left(-\phi\left(2^{n} \varphi_{n, k}\right)\right) \\
& \leq-\phi\left(\sum_{l 2^{n} \leq k<(l+1) 2^{n} .} \varphi_{n, k}\right) \\
& =-\varphi_{0, l} \log \varphi_{0, l} .
\end{aligned}
$$

Como $\int_{I_{n, k}} f(x) d x=\varphi_{n, k}$ e $\left|I_{n, k}\right|=\frac{1}{2^{n}}$, então,

$$
\begin{aligned}
-\int_{I_{n, k}} f(x) \log f(x) d x & =-\int \phi(f(x)) d x \\
& \leq-\left|I_{n, k}\right| \phi\left(\left|I_{n, k}\right|^{-1} \varphi_{n, k}\right) \\
& =-\int_{I_{n, k}} f_{n}(x) \log f_{n}(x) d x .
\end{aligned}
$$

Segue, então, dessas duas desigualdades que

$$
\begin{aligned}
-\int_{|x| \geq K} f(x) \log f(x) d x & \leq-\int_{|x| \geq K} f_{n}(x) \log f_{n}(x) d x \\
& \leq-\sum_{|k| \geq K} \varphi_{n, k} \log \varphi_{n, k} .
\end{aligned}
$$

e, fazendo $K \rightarrow \infty$, obtemos (1.22) concluindo a demonstração.

Corolário 2 Nas mesmas hipóteses do teorema acima, consideremos a variável aleatória real $Y$, cuja função densidade de probabilidade também satisfaz as propriedades (1)-(3), e a sequência de variáveis aleatórias discretas $\left(Y_{n}\right)_{n}$ definidas de modo análogo às $X_{n}^{\prime} s$. Então,

$$
\lim _{n \rightarrow \infty}\left(h\left(X_{n}\right)-h\left(Y_{n}\right)\right)=h(X)-h(Y) .
$$

Prova. Basta notarmos que:

$$
h\left(X_{n}\right)-h\left(Y_{n}\right)=\left(h\left(X_{n}\right)-n \log 2\right)-\left(h\left(Y_{n}\right)-n \log 2\right)
$$

e aplicarmos o teorema. 
Mostramos acima, através de exemplo e teorema, como a entropia contínua e discreta têm natureza não-idênticas. No entanto, como veremos nas próximas linhas ambas gozam das mesmas propriedades e, mais importante, a entropia contínua carrega o mesmo significado num certo sentido da entropia discreta como uma medida de incerteza ou informacional. Mais precisamente, a incerteza intrínseca em uma variável aleatória contínua é infinita e isso expressa-se através da igualdade $\lim _{n \rightarrow \infty} h\left(X_{n}\right)=\infty$, consequentemente, a entropia contínua não quantifica de um modo absoluto (assim como a entropia discreta) a incerteza contida nessa variável. No entanto, o que o corolário acima nos diz é que a diferença das entropias $h(X)-h(Y)$ indica a diferença entre as incertezas em $X$ e $Y$. Em outras palavras, quanto menor a diferença $h(X)-h(Y)$, mais próximas são as incertezas de $X$ e $Y$, logo, a entropia contínua fornece-nos uma medida relativa da incerteza.

Seguindo o mesmo roteiro da seção anterior definiremos agora o conceito de entropia condicional, que serve como parâmetro da incerteza sobre uma variável aleatória dado que conhecemos os resultados de uma segunda variável (a mesma alegoria usada na seção anterior, quando interpretamos uma variável aleatória como um experimento, vale aqui). Para isso, consideremos uma segunda variável aleatória $Y$ assumindo valores em $\mathbb{R}^{p}$, cuja função densidade de probabilidade é dada por $g=g(y)$ e também consideremos a função densidade de probabilidade conjunta de $X$ e $Y$ dada por $u=u(x, y)$.

Definição $4 A$ entropia condicional de $X$ dado $Y=y$ é dada por:

$$
h(X \mid Y=y):=-\int_{\mathbb{R}^{n}} \frac{u(x, y)}{g(y)} \log \frac{u(x, y)}{g(y)} d x
$$

$e$, então, definimos a entropia condicional de $X$ dado $Y$ simplesmente como a seguinte esperança:

$$
h(X \mid Y):=\int_{\mathbb{R}^{p}} h(X \mid Y=y) g(y) d y .
$$

Convém, por questão de praticidade, mantermos em mente também a expressão explícita da entropia de $X$ dado $Y$ em função apenas das funções densidade de probabilidade conjunta e de $Y$ :

$$
h(X \mid Y=y):=-\int_{\mathbb{R}^{p}} \int_{\mathbb{R}^{n}} u(x, y) \log \frac{u(x, y)}{g(y)} d x d y .
$$

Seguindo a mesma notação acima, temos o seguinte resultado:

Teorema 6

$$
h(X, Y)=h(Y)+h(X \mid Y)=h(X)+h(Y \mid X)
$$


Prova. Pela definição de entropia condicional acima:

$$
\begin{aligned}
h(X, Y) & =-\int u(x, y) \log u(x, y) d x d y=-\int u(x, y) \log (f(x \mid y) g(y)) d x d y \\
& =-\int u(x, y) \log g(y) d x d y-\int u(x, y) \log f(x \mid y) d x d y \\
& =-\int\left(\int f(x \mid y) d x\right) g(y) \log g(y) d y+\int g(y) h(X \mid Y=y) d y \\
& =h(Y)+h(X \mid Y) .
\end{aligned}
$$

A segunda igualdade demonstra-se analogamente.

Teorema 7 Sejam $f$ e $g$ funções densidade de probabilidade definidas em $\mathbb{R}^{n}$, então,

$$
h(f) \leq-\int_{\mathbb{R}^{n}} f(x) \log g(x) d x=\mathbf{E}^{f}[\log g]
$$

com igualdade se, e somente se, $f \equiv g$.

Prova. Lembremos que para todo $x>0, \log x \leq x-1$ e que a igualdade vale se, e somente se, $x=1$. Então,

$$
\begin{aligned}
h(f)+\int f(x) \log g(x) d x & =\int f(x) \log \frac{g(x)}{f(x)} d x \\
& \leq \int f(x)\left(\frac{g(x)}{f(x)}-1\right) d x \\
& =\int g(x) d x-\int f(x) d x=0
\end{aligned}
$$

com a igualdade valendo se, e somente se, $\frac{f(x)}{g(x)}=1$, para todo $x \in \mathbb{R}^{n}$, ou seja, se $f \equiv g$.

Corolário 3 Sejam $X, Y$ e $Z$ variáveis aleatórias contínuas, então,

$$
h(X \mid Y, Z) \leq h(X \mid Y) \leq h(X)
$$

onde, em primeiro lugar, $h(X \mid Y, Z)=h(X \mid Y)$ se, e somente se, $X$ e $Z$ e são independentes dado $Y$ e, em segundo lugar, $h(X \mid Y)=h(X)$ se, e somente se, $X$ e $Y$ são independentes.

Prova. Consideremos as variáveis aleatórias $Y,(Y, Z),(X, Y)$ e $(X, Y, Z)$ e suas respectivas funções densidade de probabilidade $g(y), g(y, z), f(x, y)$ e $u(x, y, z)$. 
Então,

$$
\begin{aligned}
h(X \mid Y, Z) & =-\iiint u(x, y, z) \log \frac{u(x, y, z)}{g(y, z)} d x d y d z \\
& =-\iint g(y, z)\left[\int \frac{u(x, y, z)}{g(y, z)} \log \frac{u(x, y, z)}{g(y, z)} d x\right] d y d z
\end{aligned}
$$

então, pelo teorema acima,

$$
\begin{aligned}
h(X \mid Y, Z) & \leq-\iint g(y, z)\left[\int \frac{u(x, y, z)}{g(y, z)} \log \frac{f(x, y)}{g(y)} d x\right] d y d z \\
& =-\iint\left[\int u(x, y, z) d z\right] \log \frac{f(x, y)}{g(y)} d x d y \\
& =-\iint f(x, y) \log \frac{f(x, y)}{g(y)} d x d y \\
& =h(X \mid Y)
\end{aligned}
$$

e, novamente pelo teorema acima, a igualdade ocorre se, e somente se, $\frac{u(x, y, z)}{g(y, z)} \equiv$ $\frac{f(x, y)}{g(y)}$, ou seja, se

$$
\frac{r(x, y, z)}{g(y)} \equiv \frac{f(x, y)}{g(y)} \frac{g(y, z)}{g(y)} .
$$

Em outras palavras, a igualdade ocorre se, e somente se, $Z$ é independente de $X$ dado $Y$.

Para mostrarmos a segunda parte do corolário consideremos as variáveis aleatórias $X, Y$ e $(X, Y)$ e suas respectivas funções densidade de probabilidade $f(x), g(y)$ e $u(x, y)$. Então,

$$
\begin{aligned}
h(X \mid Y) & =-\iint u(x, y) \log \frac{u(x, y)}{g(y)} d x d y \\
& =-\int g(y)\left[\int \frac{u(x, y)}{g(y)} \log \frac{u(x, y)}{g(y)} d x\right] d y
\end{aligned}
$$

então, pelo teorema acima,

$$
\begin{aligned}
h(X \mid Y) & \leq-\int g(y)\left[\int \frac{u(x, y)}{g(y)} \log f(x) d x\right] d y \\
& =-\int \log f(x)\left[\int u(x, y) d y\right] d x \\
& =-\int f(x) \log f(x) d x \\
& =h(X \mid Y)
\end{aligned}
$$

e, novamente pelo teorema acima, a igualdade ocorre se, e somente se, $\frac{u(x, y)}{g(y)} \equiv$ $f(x)$, ou seja, se, e somente se, $Y$ é independente de $X$. 


\subsubsection{Entropia Relativa}

Nesta seção definiremos o conceito de entropia relativa, também conhecida como Informação ou Divergência de Kullback-Leibler, a qual representa o núcleo de toda teoria contida nessa dissertação. Apesar de não ser uma métrica (embora sob certas circunstâncias seja possível demonstrar que a entropia relativa de fato satisfaz todas propriedades de uma distância), ela servirá exatamente para nos dar essa noção no espaço das medidas de probabilidade.

Definição 5 Sejam $\mathbf{P}$ e $\mathbf{Q}$ duas medidas de probabilidade quaisquer definidas sobre o mesmo espaço. Definimos, então, a entropia relativa, $D(\mathbf{P}, \mathbf{Q})$, entre $\mathbf{P}$ e $\mathrm{Q}$ como:

$$
D(\mathbf{P}, \mathbf{Q}):= \begin{cases}\int_{\Omega} \log \left(\frac{d \mathbf{Q}}{d \mathbf{P}}\right) d \mathbf{Q} & \text { se } \mathbf{Q} \ll \mathbf{P}, \\ \infty & \text { caso contrário. }\end{cases}
$$

obs. 1

A notação $\mathrm{Q} \ll \mathrm{P}$ significa que a medida $\mathrm{Q}$ é absolutamente contínua em relação a $\mathbf{P}$, isto é, para todo $A$ mensurável, $\mathbf{P}(A)=0$ implica $\mathbf{Q}(A)=0$.

obs. 2 Uma expressão alternativa para a entropia relativa

Se $\mathbf{P} \ll \mathbf{S}$ e $\mathbf{Q} \ll \mathbf{S}$ então (5) pode ser reescrita como:

$$
D(\mathbf{Q}, \mathbf{P})=\int_{\Omega} \frac{d \mathbf{P}}{d \mathbf{S}} \log \left(\frac{\frac{d \mathbf{P}}{d \mathbf{S}}}{\frac{d \mathbf{Q}}{d \mathbf{S}}}\right) d \mathbf{S}
$$

De fato, $\mathbf{P} \ll \mathbf{Q}$ e $\mathbf{Q} \ll \mathbf{S}$

$$
\begin{gathered}
\Rightarrow\left\{\begin{array}{l}
d \mathbf{P}(\omega)=\frac{d \mathbf{P}}{d \mathbf{Q}}(\omega) d \mathbf{Q}(\omega) \\
d \mathbf{Q}(\omega)=\frac{d \mathbf{Q}}{d \mathbf{S}}(\omega) d \mathbf{S}(\omega)
\end{array}\right. \\
\Rightarrow d \mathbf{P}(\omega)=\frac{d \mathbf{P}}{d \mathbf{Q}}(\omega) \frac{d \mathbf{Q}}{d \mathbf{S}}(\omega) d \mathbf{S}(\omega)(*)
\end{gathered}
$$

Por outro lado, $\mathbf{P} \ll \mathbf{S} \Rightarrow d \mathbf{P}(\omega)=\frac{d \mathbf{P}}{d \mathbf{S}} d \mathbf{S}$ e por unicidade, a menos de um conjunto de medida zero, temos que:

$$
\frac{d \mathbf{P}}{d \mathbf{S}}=\frac{d \mathbf{P}}{d \mathbf{S}} \frac{d \mathbf{Q}}{d \mathbf{S}}(* *) .
$$

Substituindo $(*)$ e $(* *)$ em (5) obtemos a expressão em (1.31).

Note que no caso discreto discreto a fórmula da entropia relativa para $\mathbf{p}=$ $\left(p_{1}, \ldots, p_{n}\right)$ e $\mathbf{q}=\left(q_{1}, \ldots, q_{n}\right)$ sobre um conjunto $\Omega=\left\{\omega_{1}, \ldots, \omega_{n}\right\}$ é dada por: 


$$
D(\mathbf{p}, \mathbf{q})=\sum_{i=1}^{n} q_{i} \log \frac{q_{i}}{p_{i}}
$$

Exemplo 4 Entropia relativa entre dois experimentos de Bernoulli

Seja $\Omega=\{0,1\}$ e considere as seguintes distribuições de probabilidades sobre $\Omega$ :

$$
\mathrm{q}:=(1-r, r)
$$

$\mathrm{e}$

$$
\mathbf{p}:=(1-s, s)
$$

Então,

$$
D(\mathbf{p}, \mathbf{q})=\left(\log \frac{1-r}{1-s}\right)(1-r)+\left(\log \frac{r}{s}\right) r
$$

enquanto que,

$$
D(\mathbf{q}, \mathbf{p})=\left(\log \frac{1-s}{1-r}\right)(1-s)+\left(\log \frac{s}{r}\right) s .
$$

Tomando $r=\frac{1}{2}$ e $s=\frac{1}{4}$ temos:

$$
D(\mathbf{p}, \mathbf{q})=\left(\log \frac{\frac{1}{2}}{\frac{3}{4}}\right) \frac{1}{2}+\left(\log \frac{\frac{1}{2}}{\frac{1}{4}}\right) \frac{1}{2}=0,1438 \text { nats, }
$$

$$
D(\mathbf{q}, \mathbf{p})=\left(\log \frac{\frac{3}{4}}{\frac{1}{2}}\right) \frac{3}{4}+\left(\log \frac{\frac{1}{4}}{\frac{1}{2}}\right) \frac{1}{4}=0,1308 \text { nats. }
$$

Dessa maneira, observamos que $D(\mathbf{p}, \mathbf{q}) \neq D(\mathbf{q}, \mathbf{p})$, de modo que a entropia relativa não é, em geral, simétrica.

\section{Exemplo 5 Entropia relativa entre duas distribuições binomiais}

Consideremos as duas seguintes distribuições binomiais, $\mathbf{p}:=\left(p_{0}, \ldots, p_{N}\right)$ e $\mathbf{q}:=\left(q_{0}, \ldots, q_{N}\right)$, onde $p_{j}=\left(\begin{array}{c}N \\ j\end{array}\right) p^{j}(1-p)^{N-j}$ e $q_{j}=\left(\begin{array}{c}N \\ j\end{array}\right) q^{j}(1-q)^{N-j}$ para 
$j=1, \ldots, N$. Ora, então,

$$
\begin{aligned}
D(\mathbf{p}, \mathbf{q}) & =\sum_{j=0}^{N}\left(\begin{array}{c}
N \\
j
\end{array}\right) q^{j}(1-q)^{N-j} \log \frac{\left(\begin{array}{c}
N \\
j
\end{array}\right) q^{j}(1-q)^{N-j}}{\left(\begin{array}{c}
N \\
j
\end{array}\right) p^{j}(1-p)^{N-j}} \\
& =\sum_{j=0}^{N}\left(\begin{array}{c}
N \\
j
\end{array}\right) q^{j}(1-q)^{N-j}\left(j \log \frac{q}{p}+(N-j) \log \frac{1-q}{1-p}\right) \\
& =\log \frac{q}{p} \sum_{j=0}^{N} j\left(\begin{array}{c}
N \\
j
\end{array}\right) q^{j}(1-q)^{N-j} \\
& +\log \frac{1-q}{1-p}\left(N \sum_{j=0}^{N}\left(\begin{array}{c}
N \\
j
\end{array}\right) q^{j}(1-q)^{N-j}-\sum_{j=0}^{N} j\left(\begin{array}{c}
N \\
j
\end{array}\right) q^{j}(1-q)^{N-j}\right) \\
& =\log \frac{q}{p} \sum_{j=1}^{N} j\left(\begin{array}{c}
N \\
j
\end{array}\right) q^{j}(1-q)^{N-j} \\
& +\log \frac{1-q}{1-p}\left(\begin{array}{c}
N-\sum_{j=1}^{N} j\left(\begin{array}{c}
N \\
j
\end{array}\right) q^{j}(1-q)^{N-j}
\end{array}\right)
\end{aligned}
$$

e usando a seguinte identidade:

$$
j\left(\begin{array}{c}
N \\
j
\end{array}\right)=N\left(\begin{array}{c}
N-1 \\
j-1
\end{array}\right)
$$

para $j>0$, temos,

$$
\begin{aligned}
D(\mathbf{p}, \mathbf{q}) & =\log \frac{q}{p} N \sum_{j=1}^{N}\left(\begin{array}{c}
N-1 \\
j-1
\end{array}\right) q^{j}(1-q)^{N-j} \\
& +\log \frac{1-q}{1-p}\left(N-N \sum_{j=1}^{N}\left(\begin{array}{c}
N-1 \\
j-1
\end{array}\right) q^{j}(1-q)^{N-j}\right) \\
& =N q \log \frac{q}{p} \sum_{j=1}^{N}\left(\begin{array}{c}
N-1 \\
j-1
\end{array}\right) q^{j-1}(1-q)^{(N-1)-(j-1)} \\
& +N \log \frac{1-q}{1-p}\left(\begin{array}{c}
1-q \sum_{j=1}^{N}\left(\begin{array}{c}
N-1 \\
j-1
\end{array}\right) q^{j-1}(1-q)^{(N-1)-(j-1)}
\end{array}\right)
\end{aligned}
$$

e, efetuando a troca de índices, $k:=j-1$, obtemos:

$$
\begin{aligned}
D(\mathbf{p}, \mathrm{q}) & =N q \log \frac{q}{p} \sum_{k=0}^{N-1}\left(\begin{array}{c}
N-1 \\
k
\end{array}\right) q^{k}(1-q)^{(N-1)-k} \\
& +N \log \frac{1-q}{1-p}\left(1-q \sum_{k=0}^{N-1}\left(\begin{array}{c}
N-1 \\
k
\end{array}\right) q^{k}(1-q)^{(N-1)-k}\right)
\end{aligned}
$$


ou seja,

$$
D(\mathbf{p}, \mathbf{q})=N\left(q \log \frac{q}{p}+(1-q) \log \frac{1-q}{1-p}\right)=N \cdot D(p, q) .
$$

Em outras palvras, a entropia relativa entre duas distribuições binomiais, com probabilidades de sucesso distintas, é diretamente proporcional ao número de experimentos realizados e, mais ainda, a uma razão igual ao valor da entropia relativa (ou da "distância") entre as probabilidades de sucesso de cada experimento.

No caso de distribuições de probabilidade contínuas, se $\mathbf{P}$ tem uma função densidade de probabilidade $f$ e se $\mathrm{Q}$ tem uma função densidade de probabilidade $g$, então a entropia relativa entre as duas é dada por:

$$
D(\mathbf{P}, \mathbf{Q})=\int_{\Omega} \log \left(\frac{f(x)}{g(x)}\right) f(x) d x
$$

\section{Exemplo 6 Entropia relativa entre duas distribuições normais}

Como veremos mais adiante a entropia relativa entre duas distribuições normais $\mathrm{P} \sim \mathcal{N}\left(0, \sigma^{2}\right)$ e $\mathrm{Q} \sim \mathcal{N}\left(0, \tau^{2}\right)$ é dada por:

$$
D(\mathbf{P}, \mathrm{Q})=\frac{1}{2}\left(\log \frac{\tau^{2}}{\sigma^{2}}+\frac{\sigma^{2}}{\tau^{2}}-1\right)
$$

Analogamente, se $\mathbf{S} \sim \mathcal{N}\left(0, \delta^{2}\right)$, então,

$$
D(\mathbf{Q}, \mathbf{S})=\frac{1}{2}\left(\log \frac{\delta^{2}}{\tau^{2}}+\frac{\tau^{2}}{\delta^{2}}-1\right)
$$

e

$$
D(\mathbf{P}, \mathbf{S})=\frac{1}{2}\left(\log \frac{\delta^{2}}{\sigma^{2}}+\frac{\sigma^{2}}{\delta^{2}}-1\right)
$$

$\log \mathrm{O}$

$$
\begin{aligned}
D(\mathbf{P}, \mathbf{Q})+D(\mathbf{Q}, \mathbf{S}) & =\frac{1}{2}\left\{\log \left(\frac{\delta^{2}}{\sigma^{2}}\right)+\frac{\sigma^{2}}{\tau^{2}}+\frac{\tau^{2}}{\delta^{2}}-2\right\} \\
& =\frac{1}{2}\left\{\log \left(\frac{\delta^{2}}{\sigma^{2}}\right)+\frac{\sigma^{2}}{\delta^{2}}-1-\frac{\sigma^{2}}{\delta^{2}}+\frac{\sigma^{2}}{\tau^{2}}+\frac{\tau^{2}}{\delta^{2}}-1\right\} \\
& =D(\mathbf{P}, \mathbf{S})+\frac{1}{2}\left\{\frac{\sigma^{2}}{\tau^{2}}+\frac{\tau^{2}-\sigma^{2}}{\delta^{2}}-1\right\}
\end{aligned}
$$

Tomando $\sigma=1, \tau=3$ e $\delta=2$ temos que:

$$
\frac{1}{2}\left\{\frac{\sigma^{2}}{\tau^{2}}+\frac{\tau^{2}-\sigma^{2}}{\delta^{2}}-1\right\}=\frac{5}{9}>0
$$


e portanto, neste caso, $D(\mathbf{P}, \mathbf{Q})+D(\mathbf{Q}, \mathbf{S})>D(\mathbf{P}, \mathbf{S})$.

Logo, não pode-se dizer que a entropia relativa satisfaz, em geral, a desigualdade triangular.

\subsubsection{A Distribuição Normal}

Nesta seção ilustraremos o conceito de entropia e entropia relativa calculando-as explicitamente no caso em que é dada a distribuição normal. Mostraremos que é possível caracterizar a distribuição normal em termos da entropia, mais precisamente, dado um conjunto de distribuições com uma certa matriz de covariância em comum, é a distribuição normal que maximiza a entropia. Também é possível caracterizá-la em termos da entropia relativa, já que sob certas condições (que explicitaremos mais adiante) esta a minimiza.

Definição 6 Dizemos que uma variável aleatória contínua n-dimensional $\mathbf{X}=$ $\left(X_{1}, \ldots, X_{n}\right)$ tem distribuição normal se ela admite uma função densidade de probabilidade da forma:

$$
\mathrm{p}(\mathrm{x})=\frac{1}{(2 \pi)^{\frac{d}{2}}|\Gamma|^{\frac{1}{2}}} \exp \left\{-\frac{1}{2}\left\langle\mathrm{x}-\mathrm{a}, \Gamma^{-1}(\mathrm{x}-\mathrm{a})\right\rangle\right\}
$$

onde, $\mathbf{x}, \mathbf{a} \in \mathbb{R}^{n}$ e $\Gamma:=\left(\gamma_{i j}\right)_{i, j}$ é uma matriz $n \times n$ positiva definida.

É sabido que a é vetor média de $\mathbf{X}$, ie, $\mathbf{a}=\mathbf{E}[\mathrm{X}]$ e que $\Gamma$ representa a matriz de covariância de $\mathbf{X}$, ie, $\gamma_{i j}=\mathbf{E}\left[\left(X_{i}-a_{i}\right)\left(X_{j}-a_{j}\right)\right]$ e que, portanto, é uma matriz simétrica. Note também que uma distribuição normal é completamente determinada pelos parâmetros a e $\Gamma$, de modo que, se são esses os parâmetros da distribuição de $\mathrm{X}$ denotamos esse fato por $\mathrm{X} \sim \mathcal{N}(\mathbf{a}, \Gamma)$. No caso $n=1$ a função densidade de probabilidade é dada por:

$$
p(x)=\frac{1}{\sqrt{2 \pi} \sigma} \exp \left(\frac{(x-m)^{2}}{\sigma^{2}}\right) .
$$

Aqui $\sigma^{2}$ representa a variância de $\mathrm{X}$ e escrevemos $X \sim \mathcal{N}\left(m, \sigma^{2}\right)$.

O teorema a seguir nos dá explicitamente a expressão da entropia da distribuição normal:

Proposição 1 Seja p uma função densidade de probabilidade normal com parâmetros a $e \Gamma$, então a entropia de $p$ é dada por:

$$
h(p)=\frac{1}{2} \log \left\{(2 \pi e)^{n}|\Gamma|\right\}
$$

Note que a fórmula da entropia independe da média, o que não é nada surpreendente uma vez que a entropia é uma medida de incerteza da distribuição. 
Prova. De (1.18) e de (1.35) temos que:

$$
\begin{aligned}
h(\mathbf{p}) & =\frac{1}{2} \int_{\mathbb{R}^{n}}\left\langle\mathbf{x}-\mathbf{a}, \Gamma^{-1}(\mathbf{x}-\mathbf{a})\right\rangle \mathbf{p}(\mathbf{x}) d \mathbf{x}+\log \left((2 \pi)^{\frac{n}{2}}|\Gamma|^{\frac{1}{2}}\right) \int_{\Omega} \mathbf{p}(\mathbf{x}) d \mathbf{x} \\
& =\frac{1}{2} \int_{\mathbb{R}^{n}}\left\langle\mathbf{x}-\mathbf{a}, \Gamma^{-1}(\mathbf{x}-\mathbf{a})\right\rangle \mathbf{p}(\mathbf{x}) d \mathbf{x}+\frac{1}{2} \log \left((2 \pi)^{n}|\Gamma|\right)
\end{aligned}
$$

Denotemos as componentes $\Gamma^{-1}$ por $v_{i j}$, ie, $\Gamma^{-1}=\left(v_{i j}\right)_{i, j}$. Então,

$$
\left\langle\mathbf{x}-\mathbf{a}, \Gamma^{-1}(\mathbf{x}-\mathbf{a})\right\rangle=\sum_{i, j} v_{i j}\left(x_{i}-a_{i}\right)\left(x_{j}-a_{j}\right)
$$

$\log \mathrm{O}$

$$
\begin{aligned}
\int_{\mathbb{R}^{n}}\left\langle\mathbf{x}-\mathbf{a}, \Gamma^{-1}(\mathrm{x}-\mathbf{a})\right\rangle \mathbf{p}(\mathbf{x}) d \mathbf{x} & =\sum_{i, j} v_{i j} \int_{\mathbb{R}^{n}}\left(x_{i}-a_{i}\right)\left(x_{j}-a_{j}\right) \mathbf{p}(\mathbf{x}) d \mathbf{x} \\
& =\sum_{i, j} v_{i j} \gamma_{i j}
\end{aligned}
$$

Segue da simetria de $\Gamma$ que:

$$
\begin{aligned}
\int_{\mathbb{R}^{n}}\left\langle\mathbf{x}-\mathbf{a}, \Gamma^{-1}(\mathbf{x}-\mathbf{a})\right\rangle \mathrm{p}(\mathbf{x}) d \mathbf{x} & =\sum_{i, j} v_{i j} \gamma_{j i} \\
& =\sum_{i=1}^{n}\left(\Gamma^{-1} \Gamma\right)_{i i} \\
& =t r\left(\Gamma^{-1} \Gamma\right)=n
\end{aligned}
$$

portanto,

$$
\begin{aligned}
h(\mathbf{p}) & =\frac{1}{2} n+\frac{1}{2} \log \left((2 \pi)^{n}|\Gamma|\right) \\
& =\frac{1}{2} \log e^{n}+\frac{1}{2} \log \left((2 \pi)^{n}|\Gamma|\right) \\
& =\frac{1}{2} \log \left((2 \pi e)^{n}|\Gamma|\right)
\end{aligned}
$$

obs. 3 (O Caso Unidimensional)

No caso unidimensional a fórmula (1.37) é dada por:

$$
h(p)=\frac{1}{2} \log \left(2 \pi e \sigma^{2}\right) .
$$


Aqui fica ainda mais evidente a independência da entropia com relação à média. Em contraste, a variância exerce um papel central, o que faz sentido, pois quanto maior a variância mais incerta é nossa distribuição, no sentido que torna-se mais complicado fazer previsões consistentes. Mais uma vez, isso tudo reflete o fato que a entropia é uma medida da incerteza inerente às medidas de probabilidade.

No próximo teorema calcularemos a entropia relativa da distribuição normal:

Proposição 2 Sejam $\mathbf{p} \sim \mathcal{N}(\mathbf{a}, \Gamma)$ e $\mathbf{q} \sim \mathcal{N}(\mathbf{b}, \Delta)$ funções densidade normais. Então,

$$
D(\mathbf{p}, \mathbf{q})=\frac{1}{2}\left\{\log \frac{|\Delta|}{|\Gamma|}+\operatorname{tr}\left(\Gamma\left(\Delta^{-1}+\Gamma^{-1}\right)\right)+\left\langle(\mathbf{a}-\mathbf{b}), \Delta^{-1}(\mathbf{a}-\mathbf{b})\right\rangle\right\} .
$$

Prova. Por definição,

$$
D(\mathbf{p}, \mathbf{q})=\int_{\mathbb{R}^{n}} \log \frac{\mathbf{p}(\mathbf{x})}{\mathbf{q}(\mathbf{x})} \mathbf{p}(\mathrm{x}) d \mathbf{x}
$$

então,

$$
\begin{aligned}
D(\mathbf{p}, \mathbf{q}) & =\int_{\mathbb{R}^{n}} \log (\mathrm{p}(\mathrm{x})) \mathrm{p}(\mathrm{x}) d \mathbf{x}-\int_{\mathbb{R}^{n}} \log (\mathrm{q}(\mathrm{x})) \mathbf{p}(\mathrm{x}) d \mathbf{x} \\
& =-h(\mathbf{p})-\int_{\mathbb{R}^{n}} \log (\mathbf{q}(\mathbf{x})) \mathbf{p}(\mathbf{x}) d \mathbf{x}
\end{aligned}
$$

Denotemos $\Delta^{-1}=\left(\delta_{i j}\right)_{i, j}$ e $\Gamma=\left(\gamma_{i j}\right)_{i, j}$ e calculemos a integral no termo a direita da igualdade acima:

$$
\begin{aligned}
\int_{\mathbb{R}^{n}} \log (\mathrm{q}(\mathrm{x})) \mathrm{p}(\mathrm{x}) d \mathrm{x}= & -\frac{1}{2} \int_{\mathbb{R}^{n}}\left\langle\mathrm{x}-\mathbf{b}, \Delta^{-1}(\mathrm{x}-\mathbf{b})\right\rangle \mathrm{p}(\mathrm{x}) d \mathbf{x} \\
& \frac{-}{1} 2 \int_{\mathbb{R}^{n}} \log \left((2 \pi)^{n}|\Delta|\right) \mathbf{p}(\mathbf{x}) d \mathbf{x} \\
= & -\frac{1}{2} \int_{\mathbb{R}^{n}}\left\langle\mathbf{x}-\mathbf{a}+\mathbf{a}-\mathbf{b}, \Delta^{-1}(\mathbf{x}-\mathbf{a}+\mathbf{a}-\mathbf{b})\right\rangle \mathbf{p}(\mathrm{x}) d \mathbf{x} \\
& -\frac{1}{2} \int_{\mathbb{R}^{n}} \log \left((2 \pi)^{n}|\Delta|\right) \mathbf{p}(\mathrm{x}) d \mathbf{x} \\
= & -\frac{1}{2} \int_{\mathbb{R}^{n}}\left\langle\mathbf{x}-\mathbf{a}, \Delta^{-1}(\mathbf{x}-\mathbf{a})\right\rangle \mathbf{p}(\mathbf{x}) d \mathbf{x} \\
& -\frac{1}{2} \int_{\mathbb{R}^{n}}\left\langle\mathbf{x}-\mathbf{a}, \Delta^{-1}(\mathbf{a}-\mathbf{b})\right\rangle \mathbf{p}(\mathbf{x}) d \mathbf{x} \\
& -\frac{1}{2} \int_{\mathbb{R}^{n}}\left\langle\mathbf{a}-\mathbf{b}, \Delta^{-1}(\mathbf{x}-\mathbf{a})\right\rangle \mathbf{p}(\mathbf{x}) d \mathbf{x} \\
& -\frac{1}{2} \int_{\mathbb{R}^{n}}\left\langle\mathbf{a}-\mathbf{b}, \Delta^{-1}(\mathbf{a}-\mathbf{b})\right\rangle \mathbf{p}(\mathbf{x}) d \mathbf{x} \\
& -\frac{1}{2} \log \left((2 \pi)^{n}|\Delta|\right)
\end{aligned}
$$


Repetindo o mesmo cálculo feito na demonstração do teorema (1) vemos que $-\frac{1}{2} \int_{\mathbb{R}^{n}}\left\langle\mathbf{x}-\mathbf{a}, \Delta^{-1}(\mathbf{x}-\mathbf{a})\right\rangle \mathbf{p}(\mathbf{x}) d \mathbf{x}=\operatorname{tr}\left(\Gamma \Delta^{-1}\right), \log \mathrm{o}$,

$$
\begin{aligned}
\int_{\mathbb{R}^{n}} \log (\mathrm{q}(\mathbf{x})) \mathbf{p}(\mathbf{x}) d \mathbf{x}= & -\frac{1}{2} \operatorname{tr}\left(\Gamma \Delta^{-1}\right) \\
& -\frac{1}{2} \int_{\mathbb{R}^{n}} \sum_{i, j} \delta_{i j}\left(x_{i}-a_{i}\right)\left(a_{j}-b_{j}\right) \mathbf{p}(\mathbf{x}) d \mathbf{x} \\
& -\frac{1}{2} \int_{\mathbb{R}^{n}} \sum_{i, j} \delta_{i j}\left(x_{j}-a_{j}\right)\left(a_{i}-b_{i}\right) \mathbf{p}(\mathbf{x}) d \mathbf{x} \\
& -\frac{1}{2}\left\langle\mathbf{a}-\mathbf{b}, \Delta^{-1}(\mathbf{a}-\mathbf{b})\right\rangle-\frac{1}{2} \log \left((2 \pi)^{n}|\Delta|\right) \\
= & -\frac{1}{2} \operatorname{tr}\left(\Gamma \Delta^{-1}\right)-\frac{1}{2} \log \left((2 \pi)^{n}|\Delta|\right) \\
& -\frac{1}{2}\left\langle\mathbf{a}-\mathbf{b}, \Delta^{-1}(\mathbf{a}-\mathbf{b})\right\rangle
\end{aligned}
$$

Por outro lado, pelo teorema (1) temos que:

$$
\begin{aligned}
h(\mathbf{p}) & =\frac{1}{2} \log \left\{(2 \pi e)^{n}|\Gamma|\right\}=\frac{1}{2} \log \left\{(2 \pi)^{n}|\Gamma|\right\}+\frac{n}{2} \\
& =\frac{1}{2} \log \left\{(2 \pi)^{n}|\Gamma|\right\}+\frac{1}{2} \operatorname{tr}\left(\Gamma \Gamma^{-1}\right)
\end{aligned}
$$

Portanto,

$$
\begin{aligned}
h(\mathbf{p})= & -\frac{1}{2} \log \left\{(2 \pi)^{n}|\Gamma|\right\}-\frac{1}{2} \operatorname{tr}\left(\Gamma \Gamma^{-1}\right)+\frac{1}{2} \operatorname{tr}\left(\Gamma \Delta^{-1}\right)+\frac{1}{2} \log \left((2 \pi)^{n}|\Delta|\right) \\
& +\frac{1}{2}\left\langle\mathbf{a}-\mathbf{b}, \Delta^{-1}(\mathbf{a}-\mathbf{b})\right\rangle \\
= & \frac{1}{2} \log \left(\frac{|\Delta|}{|\Gamma|}\right)+\frac{1}{2} \operatorname{tr}\left(\Gamma\left(\Delta^{-1}-\Gamma^{-1}\right)\right)+\frac{1}{2}\left\langle\mathbf{a}-\mathbf{b}, \Delta^{-1}(\mathbf{a}-\mathbf{b})\right\rangle
\end{aligned}
$$

o que demonstra a proposição.

Em particular, no caso unidimensional, se $p \sim \mathcal{N}\left(a, \sigma^{2}\right)$ e $q \sim \mathcal{N}\left(b, \tau^{2}\right)$, a fórmula (1.39) é dada por:

$$
D(p, q)=\frac{1}{2}\left(\log \frac{\tau^{2}}{\sigma^{2}}+\frac{\sigma^{2}}{\tau^{2}}-1+\frac{(a-b)^{2}}{\tau^{2}}\right)
$$

Agora, mostraremos como a entropia pode caracterizar uma distribuição normal. De fato, considerando todas distribuições de probabilidade com matriz de covariância e média dadas, a distribuição normal é aquela que maximiza a entropia. 
Teorema 8 Seja $f$ uma função densidade de probabilidade $\mathcal{N}(\mathbf{a}, \Gamma)$ e $g(x)$ uma outra função densidade de probabilidade cuja matriz de covariância também é dada por $\Gamma$. Então,

$$
h(g) \leq h(f) .
$$

Prova. De fato, usando o teorema (7) temos

$$
\begin{aligned}
h(g) & \leq-\int g(\mathbf{x}) \log f(\mathbf{x}) d \mathbf{x} \\
& =-\int g(\mathbf{x}) \log \frac{1}{(2 \pi)^{\frac{d}{2}}|\Gamma|^{\frac{1}{2}}} \exp \left\{-\frac{1}{2}\left\langle\mathbf{x}-\mathbf{a}, \Gamma^{-1}(\mathbf{x}-\mathbf{a})\right\rangle\right\} d \mathbf{x} \\
& =\int g(\mathbf{x}) \log \left[(2 \pi)^{\frac{d}{2}}|\Gamma|^{\frac{1}{2}}\right] d \mathbf{x}+\frac{1}{2} \int g(\mathbf{x})\left\langle\mathbf{x}-\mathbf{a}, \Gamma^{-1}(\mathbf{x}-\mathbf{a})\right\rangle d \mathbf{x} \\
& =\log \left[(2 \pi)^{\frac{d}{2}}|\Gamma|^{\frac{1}{2}}\right]+\frac{1}{2} \int f(\mathbf{x})\left\langle\mathbf{x}-\mathbf{a}, \Gamma^{-1}(\mathbf{x}-\mathbf{a})\right\rangle d \mathbf{x}
\end{aligned}
$$

sendo a última igualdade verdadeira pois as matrizes de covariância de $f$ e $g$ são iguais. Logo,

$$
\begin{aligned}
h(g) & \leq \int \log \left[(2 \pi)^{\frac{d}{2}}|\Gamma|^{\frac{1}{2}}\right] f(\mathbf{x}) d \mathbf{x}+\frac{1}{2} \int f(\mathbf{x})\left\langle\mathbf{x}-\mathbf{a}, \Gamma^{-1}(\mathbf{x}-\mathbf{a})\right\rangle d \mathbf{x} \\
& =-\int f(\mathbf{x}) \log \frac{1}{(2 \pi)^{\frac{d}{2}}|\Gamma|^{\frac{1}{2}}} \exp \left\{-\frac{1}{2}\left\langle\mathbf{x}-\mathbf{a}, \Gamma^{-1}(\mathbf{x}-\mathbf{a})\right\rangle\right\} d \mathbf{x} \\
& =h(f)
\end{aligned}
$$

como queríamos demonstrar.

Observemos pela demonstração acima que não importa a média sob ambas funções densidade de probabilidade, ou seja, dada qualquer função densidade de probabilidade, sua entropia é superiormente limitada pela distribuição normal cuja matriz de covariância seja igual a sua.

Um outro modo de caracterizar a entropia relativa é usando o conceito de entropia relativa no sentido de que dada uma medida de referência gaussiana qualquer, então dentre todas funções densidade de probabilidade com mesma média e matriz de covariância, aquela que minimiza a entropia relativa é exatamente a distribuição normal.

Teorema 9 Sejam $f$ e $g$ funções densidade de probabilidade normais $\mathcal{N}(\mathbf{a}, \Delta)$ e $\mathcal{N}(\mathrm{b}, \Gamma)$, então dada qualquer função densidade de probabilidade $r$ com média e matriz de covariância dados por $\mathbf{b}$ e $\Gamma$ temos

$$
D(f, g) \leq D(f, r) .
$$

Prova. Pelo teorema 11 temos

$$
D(f, g)=-h(g)-\int g(\mathbf{x}) \log f(\mathbf{x}) d \mathbf{x}
$$




$$
D(f, r)=-h(r)-\int r(\mathbf{x}) \log f(\mathbf{x}) d \mathbf{x}
$$

Agora observando a identidade (1.40) na demonstração do teorema 2 e lembrando que, por hipótese, $g$ e $r$ têm a mesma média e mesma matriz de covariância temos

$$
\int g(\mathbf{x}) \log f(\mathbf{x}) d \mathbf{x}=\int r(\mathbf{x}) \log f(\mathbf{x}) d \mathbf{x} .
$$

E como pelo teorema anterior

$$
-h(g) \leq-h(r)
$$

então,

$$
D(f, g) \leq D(f, r)
$$

como queríamos demonstrar.

\subsubsection{Propriedades da Entropia Relativa}

Apesar da entropia relativa não ser efetivamente uma métrica como vimos pelos exemplos acima, ela satisfaz uma de suas propriedades. Porém antes de a enunciar e provar, consideremos o seguinte lema:

Lema 2 Para todo $x>0$ temos:

$$
\log x \leq x-1 .
$$

Prova. Consideremos a função $f(x):=x-\log x$. Em primeiro lugar, como - $\log x$ é uma função convexa, segue que $f$ também o é, logo, se $f$ tem um ponto crítico, este, obrigatoriamente, é um ponto de mínimo global. Ora, $f^{\prime}(x)=$ $1-\frac{1}{x}=0$ se, e somente se, $x=1$. Portanto, 1 é um mínimo global de $f$, ou seja, $1=f(1) \leq f(x)=x-\log x$, para todo $x>0$. Em outras palavras $\log x \leq x-1$, para qualquer $x$ positivo.

Teorema 10 Sejam $\mathbf{P}$ e $\mathbf{Q}$ medidas de probabilidade sobre $(\Omega, \mathcal{B}(\Omega))$. Então, $D(\mathbf{P}, \mathbf{Q}) \geq 0$, com igualdade se, e somente se, $\mathbf{P}=\mathbf{Q}$.

\section{Prova.}

O resultado é óbvio se $\mathbf{Q}$ não é absolutamente contínua em relação a $\mathbf{P}$, consequentemente, podemos, sem perda de generalidade, assumir que $\mathbf{Q} \ll \mathbf{P}$ e utilizando o lema anterior temos: 


$$
\begin{aligned}
D(\mathbf{P}, \mathbf{Q}) & =\int_{\Omega} \log \left(\frac{d \mathbf{Q}}{d \mathbf{P}}(x)\right) d \mathbf{Q}(x) \\
& \geq \int_{\Omega}\left(1-\frac{d \mathbf{P}}{d \mathbf{Q}}(x)\right) d \mathbf{Q}(x) \\
& =\int_{\Omega} d \mathbf{Q}(x)-\int_{\Omega} d \mathbf{P}(x)=0
\end{aligned}
$$

Como no lema acima vale a igualdade se, e somente se, $x=1$, segue que $D(\mathbf{P}, \mathbf{Q})=0$ se, e somente se, $\frac{d \mathbf{Q}}{d \mathbf{P}}(x)=1$ para $\mathbf{Q}$-q.t.p, o que equivale a $\mathbf{P}=\mathbf{Q}$.

Uma questão que nos interessa é saber qual a relação, se existir, entre entropia e entropia relativa: A importância dessa relação reside nos problemas de minimização da entropia relativa e de maximização da entropia, os quais consideraremos mais adiante. Quanto a questão da existência a resposta é afirmativa e exibiremos logo abaixo a relação entre ambas.

Teorema 11 Há dois casos a considerarmos:

1. Caso discreto. Consideremos as distribuições de probabilidades $\mathbf{p}=$ $\left(p_{1}, \ldots, p_{n}\right)$ e $\mathbf{q}=\left(q_{1}, \ldots, q_{n}\right)$, então,

$$
h(\mathbf{q})=-D(\mathbf{p}, \mathbf{q})-\sum_{i=1}^{n} q_{i} \log p_{i} .
$$

Em particular, se $\mathbf{p}=\mathbf{u}=\left(\frac{1}{n}, \ldots, \frac{1}{n}\right)$ é a distribuição uniforme, então,

$$
h(\mathbf{q})=-D(\mathbf{u}, \mathbf{q})+\log n .
$$

2. Caso contínuo. Consideremos agora as seguintes funções densidade de probabilidade $f$ e $g$, então,

$$
h(g)=-D(f, g)-\int g(x) \log f(x) d x .
$$

Em particular, se $f(x)=u(x)=\left\{\begin{array}{ll}\frac{1}{|\Delta|} & \text { se } x \in \Delta, \\ 0 & \text { caso contrário }\end{array}\right.$, onde $\Delta$ é um intervalo limitado qualquer, então,

$$
h(g)=-D(u, g)+\log |\Delta| .
$$

Prova. As demonstrações, nos dois casos, são análogas e imediatas, portanto, faremos apenas a prova do segundo caso. Ora,

$$
\begin{aligned}
D(f, g) & =\int g(x) \log \frac{g(x)}{f(x)} d x=\int g(x) \log g(x) d x-\int g(x) \log f(x) d x \\
& =-h(f)-\int g(x) \log f(x) d x
\end{aligned}
$$


o que demonstra a primeira afirmação do segundo caso. Se substituimos $f(x)$ por $u(x)$, definida no enunciado, e usamos a afirmação que acabamos de demonstrar obtemos:

$$
h(g)=-D(u, g)-\int_{\Delta} g(x) \log \frac{1}{|\Delta|} d x=-D(u, g)+\log |\Delta|
$$

como queríamos demosntrar.

O próximo resultado nos dá uma idéia do que ocorre quando procuramos minimizar a entropia relativa em relação a uma distribuição de probabilidade dada a qual denominamos distribuição de referência (ou 'prior'). Como veremos mais adiante, se nenhuma restrição é imposta o problema de minimizar a entropia relativa é trivial pois ela é maior ou igual a zero e $D(\mathbf{p}, \mathbf{p})=0$. Porém, quando restrições são impostas isso já pode não ocorrer, pois, $\mathbf{p}$ pode não satisfazer tais restrições. Sendo assim, a proposição abaixo mostra como p se "move" em direção a distribuição ótima.

Proposição 3 Sejam $\mathbf{p}$ e q distribuições discretas de probabilidade e suponhamos que $\mathbf{p}>0$. Então, temos a seguinte aproximação para a entropia relativa

$$
D(\mathbf{p}, \mathbf{q})=\sum_{k} q_{k} \log \frac{q_{k}}{p_{k}} \approx \sum_{k} \frac{1}{p_{k}}\left(q_{k}-p_{k}\right)^{2} .
$$

Prova. Usando série de Taylor

$$
\log \frac{q_{k}}{p_{k}}=\log \left(1+\frac{q_{k}-p_{k}}{p_{k}}\right)=\frac{q_{k}-p_{k}}{p_{k}}+O\left(\left(\frac{q_{k}-p_{k}}{p_{k}}\right)^{2}\right) .
$$

Assumindo que $\left|\frac{q_{k}-p_{k}}{p_{k}}\right|$ é pequeno, uma boa aproximação para $\log \frac{q_{k}}{p_{k}}$ é $\frac{q_{k}-p_{k}}{p_{k}}$. Agora,

$$
\begin{aligned}
\sum_{k} q_{k} \frac{q_{k}-p_{k}}{p_{k}} & =\sum_{k} \frac{q_{k}^{2}-p_{k} q_{k}}{p_{k}}+\sum_{k} p_{k}-\sum_{k} q_{k} \\
& =\sum_{k} \frac{\left(q_{k}-p_{k}\right)^{2}}{p_{k}} .
\end{aligned}
$$

Ou seja,

$$
D(\mathbf{p}, \mathbf{q}) \approx \sum_{k} \frac{\left(q_{k}-p_{k}\right)^{2}}{p_{k}}
$$

A partir da fórmula (1.47) podemos observar que para uma distribuição de probabilidade q fixa menos provável é um estado, ou seja, quanto menor a 
probabilidade de ocorrência $p_{k}$ desse estado mais ele contribui para que a magnitude da entropia relativa seja grande. Portanto, a distribuição ótima deve concentrar menos massa onde já havia pouca probabilidade de ocorrência e aumentar onde já havia muita. Portanto, ao minimizarmos a entropia relativa estamos deformando a distribuição de referência concedendo mais peso aos estados inicialmente mais prováveis e menos peso aos estados inicialmente menos prováveis.

Lema 3 Sejam $a_{i}, b_{i} \geq 0$ para $i=1, \ldots, m$ tais que $\sum_{i=1}^{m} a_{i}>0$ e $\sum_{i=1}^{m} b_{i}>0$. Então,

$$
\sum_{i=1}^{m} a_{i} \log \frac{a_{i}}{b_{i}} \geq\left(\sum_{i=1}^{m} a_{i}\right) \log \frac{\sum_{i=1}^{m} a_{i}}{\sum_{i=1}^{m} b_{i}} .
$$

A igualdade vale se, e somente se, $\frac{a_{1}}{b_{1}}=\ldots=\frac{a_{m}}{b_{m}}$.

Prova. Notemos que $q=\left(\frac{a_{1}}{a}, \ldots, \frac{a_{m}}{a}\right)$ e $p=\left(\frac{b_{1}}{b}, \ldots, \frac{b_{m}}{b}\right)$, onde $a=\sum_{i=1}^{m} a_{i}$ e $b=\sum_{i=1}^{m} b_{i}$, são distribuiçôes de probabilidade e, portanto

$$
D(p, q)=\sum_{i=1}^{m} \frac{a_{i}}{a} \log \frac{\sum_{i=1}^{m} a_{i}}{\sum_{i=1}^{m} b_{i}} \geq 0
$$

ou seja $\frac{1}{a} \sum_{i=1}^{m} a_{i} \log \frac{b}{a} \frac{a_{i}}{b_{i}} \geq 0, \log \mathrm{O}$

$$
\sum_{i=1}^{m} a_{i} \log \frac{b}{a}+\sum_{i=1}^{m} a_{i} \log \frac{a_{i}}{b_{i}} \geq 0
$$

e então

$$
\sum_{i=1}^{m} a_{i} \log \frac{a_{i}}{b_{i}} \geq \sum_{i=1}^{m} a_{i} \log \frac{a}{b} .
$$

Além disso, a igualdade vale se, e somente se, $p_{i}=q_{i}$ para todo $i=1, \ldots, m$, isto é, se, e somente se, $\frac{a_{1}}{b_{1}}=\ldots=\frac{a_{m}}{b_{m}}$ para todo $i=1, \ldots, m$.

Teorema 12 A entropia relativa $D(\mathbf{P}, \mathbf{Q})$ é convexa em $(\mathbf{P}, \mathbf{Q})$, ou seja, se $\mathbf{P}=\alpha \mathbf{P}_{1}+(1-\alpha) \mathbf{P}_{\mathbf{2}}$ e $\mathbf{Q}=\alpha \mathbf{Q}_{\mathbf{1}}+(1-\alpha) \mathbf{Q}_{\mathbf{2}}$ para $\alpha \in[0,1]$, então

$$
D(\mathbf{P}, \mathbf{Q}) \leq \alpha D\left(\mathbf{P}_{\mathbf{1}}, \mathbf{Q}_{\mathbf{1}}\right)+(1-\alpha) D\left(\mathbf{P}_{\mathbf{2}}, \mathbf{Q}_{\mathbf{2}}\right) \text {. }
$$

Prova. Se $P_{1}$ não é absolutamente contínua em relação a $Q_{1}$ ou $P_{2}$ não é absolutamente contínua em relação a $\mathbf{Q}_{2}$ a desigualdade é trivial, portanto, podemos assumir que $\mathbf{P}_{\mathbf{1}} \ll \mathrm{Q}_{1}$ e $\mathbf{P}_{\mathbf{2}} \ll \mathbf{Q}_{\mathbf{2}}$. Então

$$
\begin{aligned}
D(\mathbf{P}, \mathbf{Q}) & =\int \log \frac{d \mathbf{Q}}{d \mathbf{P}} d \mathbf{Q} \\
& =\int \frac{d \mathbf{Q}}{d \mathbf{P}} \log \frac{\alpha d \mathbf{Q}_{1}+(1-\alpha) d \mathbf{Q}_{2}}{\alpha d \mathbf{P}_{1}+(1-\alpha) d \mathbf{P}_{2}} d \mathbf{P} \\
& =\int \log \frac{\alpha \frac{d \mathbf{Q}_{1}}{d \mathbf{P}}+(1-\alpha) \frac{d \mathbf{Q}_{2}}{d \mathbf{P}_{2}}}{\alpha \frac{\mathbf{P}_{2}}{d \mathbf{P}}+(1-\alpha) \frac{d \mathbf{P}_{2}}{d \mathbf{P}}} \log \left(\frac{\alpha \frac{d \mathbf{Q}_{1}}{d \mathbf{P}_{1}}+(1-\alpha) \frac{d \mathbf{Q}_{2}}{d \mathbf{P}}}{\alpha \frac{\mathbf{P}_{1}}{d \mathbf{P}}+(1-\alpha) \frac{d \mathbf{P}_{2}}{d \mathbf{P}}}\right) d \mathbf{P}_{2}
\end{aligned}
$$


e, portanto, de acordo com o lema 3

$$
\begin{aligned}
D(\mathbf{P}, \mathbf{Q}) & \leq \alpha \int \frac{d \mathbf{Q}_{\mathbf{1}}}{d \mathbf{P}} \log \frac{d \mathbf{Q}_{1}}{d \mathbf{P}_{\mathbf{1}}} d \mathbf{P}+(1-\alpha) \int \frac{d \mathbf{Q}_{\mathbf{2}}}{d \mathbf{P}} \log \frac{d \mathbf{Q}_{2}}{d \mathbf{P}_{\mathbf{2}}} d \mathbf{P} \\
& =\alpha D\left(\mathbf{P}_{\mathbf{1}}, \mathbf{Q}_{\mathbf{1}}\right)+(1-\alpha) D\left(\mathbf{P}_{\mathbf{2}}, \mathbf{Q}_{\mathbf{2}}\right)
\end{aligned}
$$

como queríamos demonstrar.

Teorema 13 A entropia relativa $D(\mathbf{P}, \mathbf{Q})$ é estritamente convexa em $\mathbf{Q}$, ou seja, se $\mathbf{Q}=\alpha \mathbf{Q}_{1}+(1-\alpha) \mathbf{Q}_{2}, \mathbf{Q}_{1} \neq \mathbf{Q}_{2}, D\left(\mathbf{P}, \mathbf{Q}_{1}\right)<\infty, D\left(\mathbf{P}, \mathbf{Q}_{2}\right)<\infty e$ $\alpha \in[0,1]$, então

$$
D(\mathbf{P}, \mathbf{Q}) \leq \alpha D\left(\mathbf{P}, \mathbf{Q}_{1}\right)+(1-\alpha) D\left(\mathbf{P}, \mathbf{Q}_{2}\right) .
$$

Prova.

$$
\begin{aligned}
D(\mathbf{P}, \mathbf{Q}) & =\int \log \left(\frac{\alpha d \mathbf{Q}_{1}+(1-\alpha) d \mathbf{Q}_{2}}{d \mathbf{P}}\right)\left(\alpha \frac{d \mathbf{Q}_{1}}{d \mathbf{P}}+(1-\alpha) \frac{d \mathbf{Q}_{2}}{d \mathbf{P}}\right) d \mathbf{P} \\
& =\int \log \left(\frac{\alpha \frac{d \mathbf{Q}_{1}}{d \mathbf{P}}+(1-\alpha) \frac{d \mathbf{Q}_{2}}{d \mathbf{P}}}{\alpha \frac{d \mathbf{P}}{d \mathbf{P}}+(1-\alpha) \frac{d \mathbf{P}}{d \mathbf{P}}}\right)\left(\alpha \frac{d \mathbf{Q}_{\mathbf{1}}}{d \mathbf{P}}+(1-\alpha) \frac{d \mathbf{P}}{d \mathbf{P}}\right) d \mathbf{P}
\end{aligned}
$$

e, consequentemente, pelo lema 3

$$
\begin{aligned}
D(\mathbf{P}, \mathbf{Q}) & <\alpha \int \log \frac{d \mathbf{Q}_{\mathbf{1}}}{d \mathbf{P}} d \mathbf{Q}_{\mathbf{1}}+(1-\alpha) \frac{d \mathbf{Q}_{\mathbf{2}}}{d \mathbf{P}} d \mathbf{Q}_{\mathbf{2}} \\
& =\alpha D\left(\mathbf{P}, \mathbf{Q}_{\mathbf{1}}\right)+(1-\alpha) D\left(\mathbf{P}, \mathbf{Q}_{\mathbf{2}}\right)
\end{aligned}
$$

sendo a desigualdade estrita pois pelas nossas hipóteses

$$
\frac{\alpha \frac{d \mathbf{Q}_{1}}{d \mathrm{P}}}{\alpha \frac{d \mathrm{P}}{d \mathrm{P}}}=\frac{d \mathbf{Q}_{1}}{d \mathbf{P}} \neq \frac{d \mathbf{Q}_{2}}{d \mathbf{P}}=\frac{\alpha \frac{d \mathbf{Q}_{2}}{d \mathrm{P}}}{\alpha \frac{d \mathrm{P}}{d \mathrm{P}}} .
$$

\subsubsection{A Desigualdade de Csiszár}

Os resultados que seguem serão úteis mais a frente quando formos deduzir a representação funcional da entropia relativa, no entanto, eles também justificam, em parte, chamarmos a entropia relativa de métrica.

Seja $\Omega$ um conjunto qualquer. Dizemos que

$$
\Delta=\left\{A_{1}, \ldots, A_{n}\right\}
$$

é uma partição de $\Omega$ se os $A_{i}$ 's são dois a dois disjuntos e $\Omega=\bigcup_{i} A_{i}$. Dizemos que $\Gamma$ é um refinamento da partição $\Delta$ se $\Gamma$ também é uma partição de $\Omega$ e para 
todo $B_{j} \in \Gamma$ existe $A_{i} \in \Delta$ tal que $B_{j} \subset A_{i}$. Denotemos por $\Pi$ o conjunto de todas partições de $\Omega$.

Dada uma partição de $\Omega$ é possível, num certo sentido, discretizar a entropia relativa. Para tal definimos o conceito de entropia relativa sobre uma partição. Mais ainda, é possível mostrar que a entropia relativa entre duas medidas de probabilidade é igual ao supremo, sobre todas partições de $\Omega$, das entropias relativas sobre uma partição destas mesmas medidas de probabilidade.

Definição 7 Dadas duas medidas de probabilidade $\mathbf{P}$ e $\mathbf{Q}$ sobre $(\Omega, \mathcal{B}(\Omega))$ e dada uma partição $\Delta=\left\{A_{1}, \ldots, A_{m}\right\} \in \Pi$ definimos a entropia relativa de $\mathrm{Q}$ em relação a $\mathbf{P}$ sobre a partição $\Delta$ por:

$$
D_{\Delta}(\mathbf{P}, \mathbf{Q})=\sum_{i=1}^{m} \mathrm{Q}\left(A_{i}\right) \log \left(\frac{\mathrm{Q}\left(A_{i}\right)}{\mathrm{P}\left(A_{i}\right)}\right)
$$

Suponha que $\mathbf{Q}$ não é absolutamente contínua em relação a $\mathbf{P}$. Então, existe $A \in \mathcal{B}(\Omega)$ tal que $\mathbf{P}(A)=0$, mas $\mathbf{Q}(A)>0$, de modo que podemos considerar a partição $\left\{A, A^{C}\right\}$, de onde

$$
D_{\Delta}(\mathbf{P}, \mathbf{Q})=\mathbf{Q}(A) \log \left(\frac{\mathrm{Q}(A)}{\mathbf{P}(A)}\right)+\mathbf{Q}\left(A^{C}\right) \log \left(\frac{\mathrm{Q}\left(A^{C}\right)}{\mathbf{P}\left(A^{C}\right)}\right)=\infty .
$$

Logo, neste caso $D(\mathbf{P}, \mathbf{Q})=D_{\Delta}(\mathrm{P}, \mathrm{Q})$. Assumamos agora que $\mathrm{Q} \ll \mathbf{P}$ e seja $\frac{d \mathbf{Q}}{d \mathbf{P}}$ a derivada de Radón-Nikodyn de $\mathbf{Q}$ em relação a $\mathbf{P}$. Seja $\Delta=\left\{A_{1}, \ldots, A_{m}\right\}$ uma partição qualquer de $\Omega$, e definamos

$$
\mathbf{P}^{\Delta}(A)=\int_{A} \chi^{\Delta}(x) d \mathbf{P}(x)
$$

onde $\chi^{\Delta}(x)=\sum_{k=1}^{m} \frac{\mathbf{Q}\left(A_{k}\right)}{\mathrm{P}\left(A_{k}\right)} \mathbf{1}_{\mathbf{A}_{\mathbf{k}}}(x)$.

Além disso, é verdade que $D_{\Delta}(\mathrm{P}, \mathrm{Q})$ é a esperança de $\log \chi^{\Delta} \operatorname{com}$ relação à medida $\mathrm{Q}$, de fato,

$$
\begin{aligned}
D_{\Delta}(\mathbf{P}, \mathbf{Q}) & =\sum_{k=1}^{m} \mathbf{Q}\left(A_{k}\right) \log \frac{\mathbf{Q}\left(A_{k}\right)}{\mathbf{P}\left(A_{k}\right)} \\
& =\sum_{k=1}^{m} \log \frac{\mathbf{Q}\left(A_{k}\right)}{\mathbf{P}\left(A_{k}\right)} \int_{A_{k}} d \mathbf{Q}(x)
\end{aligned}
$$

e, portanto,

$$
D_{\Delta}(\mathbf{P}, \mathbf{Q})=\int_{\Omega} \log \chi^{\Delta}(x) d \mathbf{Q}(x)
$$


Mais ainda, é verdade que $\mathrm{Q} \ll \mathrm{Q}^{\Delta}$, pois

$$
\begin{aligned}
\mathrm{P}^{\Delta}=0 & \Rightarrow \int_{A} \chi^{\Delta}(x) d \mathbf{P}(x)=0 \\
& \Rightarrow \exists E \in \mathcal{B}(\Omega): A=E \cup E^{c}, \mathbf{P}(E)=\left.0 e \chi^{\Delta}\right|_{E^{c}} \equiv 0 \\
& \Rightarrow \mathrm{Q}(E)=0 \text { pois } \mathbf{Q} \ll \mathbf{P} e \frac{\mathbf{Q}\left(E^{c}\right)}{\mathbf{P}\left(E^{c}\right)}=0 \\
& \Rightarrow \mathrm{Q}(E)=0 e \mathbf{Q}\left(E^{c}\right)=0, \text { portanto, } \mathrm{Q}(A)=0 \\
& \Rightarrow \mathrm{Q} \ll \mathbf{P}^{\Delta} .
\end{aligned}
$$

Então, faz sentido falar na derivada de Radon-Nikodyn: $\frac{d \mathbf{Q}}{d \mathrm{P}^{\Delta}}(x)$ e vale a seguinte relação:

$$
\frac{d \mathbf{Q}}{d \mathbf{P}^{\Delta}}(x)=\frac{1}{\chi^{\Delta}(x)} \frac{d \mathbf{Q}}{d \mathbf{P}}(x)
$$

De fato,

$$
\begin{aligned}
\mathbf{P}^{\Delta}(A) & =\int_{A} \chi^{\Delta}(x) d \mathbf{P}(x) \\
& \Rightarrow \mathbf{P}^{\Delta} \ll \mathbf{P} e d \mathbf{P}^{\Delta}(x)=\chi^{\Delta}(x) d \mathbf{P}(x)
\end{aligned}
$$

$\log \mathrm{O}$

$$
\begin{aligned}
\mathbf{Q}(A) & =\int_{A} \frac{d \mathbf{Q}}{d \mathbf{P}^{\Delta}}(x) d \mathbf{P}^{\Delta}(x) \\
& =\int_{A} \frac{d \mathbf{Q}}{d \mathbf{P}^{\Delta}}(x) \chi^{\Delta}(x) d \mathbf{P}(x)
\end{aligned}
$$

por outro lado,

$$
\mathbf{Q}(A)=\int_{A} \frac{d \mathbf{Q}}{d \mathbf{P}}(x) d \mathbf{P}(x)
$$

de maneira que,

$$
\frac{d \mathbf{Q}}{d \mathbf{P}^{\Delta}}(x) \chi^{\Delta}(x)=\frac{d \mathbf{Q}}{d \mathbf{P}}(x) .
$$

Então, aplicando essa relação na fórmula da entropia relativa obtemos:

$$
\begin{aligned}
D\left(\mathbf{P}^{\Delta}, \mathbf{Q}\right) & =\int_{\Omega} \log \frac{d \mathbf{Q}}{d \mathbf{P}^{\Delta}}(x) d \mathbf{Q}(x) \\
& =\int_{\Omega} \log \left(\frac{1}{\chi^{\Delta}(x)} \frac{d \mathbf{Q}}{d \mathbf{P}}(x)\right) d \mathbf{Q}(x) \geq 0
\end{aligned}
$$

pois a entropia relativa é sempre não negativa. Logo,

$$
\int_{\Omega} \log \frac{d \mathbf{Q}}{d \mathbf{P}}(x) d \mathbf{Q}(x) \geq \int_{\Omega} \log \chi^{\Delta}(x) d \mathbf{Q}(x)
$$


ou seja:

$$
D(\mathbf{P}, \mathbf{Q}) \geq D_{\Delta}(\mathbf{P}, \mathbf{Q}) .
$$

Como a partição $\Delta$ foi tomada arbitrariamente, segue que:

$$
D(\mathbf{P}, \mathbf{Q}) \geq \sup _{\Delta \in \Pi} D_{\Delta}(\mathbf{P}, \mathbf{Q}) .
$$

Enunciemos, agora, essa desigualdade na forma de um lema:

Lema 4 Sejam $\mathbf{P}$ e $\mathbf{Q}$ medidas de probabilidade arbitrárias sobre $(\Omega, \mathcal{B}(\mathbf{P}))$. Então,

$$
D(\mathbf{P}, \mathbf{Q}) \geq \sup _{\Delta \in \Pi} D_{\Delta}(\mathbf{P}, \mathbf{Q})
$$

onde $\Pi$ é o conjunto de todas partições de $\Omega$.

O lema acima será usado mais adiante na demonstração da desigualdade de Csiszár, a qual desempenha um papel central e, portanto, nos ateremos a ela agora.

Definição 8 Dizemos que uma sequência de medidas de probabilidade $\left\{\mathbf{P}_{\mathbf{n}}\right\}_{n}$ converge em entropia para $\mathbf{P}$ se

$$
\lim _{n \rightarrow \infty} D\left(\mathbf{P}, \mathbf{P}_{\mathbf{n}}\right)=0
$$

e escrevemos $\mathbf{P}_{\mathbf{n}} \rightarrow^{\varepsilon} \mathbf{P}$.

Se $\mathrm{Q}$ é uma outra medida de probabilidade, definimos a variação total de $\mathrm{P}$ e $\mathrm{Q}$ por:

$$
\|\mathbf{P}-\mathbf{Q}\|:=\sup _{\Delta \in \Pi} \sum_{i=1}^{m}\left|\mathbf{P}\left(A_{i}\right)-\mathrm{Q}\left(A_{i}\right)\right| .
$$

Notemos que ||.|| define uma métrica sobre o espaço das medidas de probabilidade $\mathbf{P}$. Dizemos, então, que $\left\{\mathbf{P}_{\mathbf{n}}\right\}$ converge $\mathbf{e m}$ variação para $\mathbf{P}$ se

$$
\lim _{n \rightarrow \infty}\left\|\mathbf{P}_{\mathbf{n}}-\mathbf{P}\right\|=0 .
$$

Neste caso, temos para todo $A \in \mathcal{B}(\Omega)$ a igualdade

$$
\lim _{n \rightarrow \infty} \mathbf{P}_{\mathbf{n}}(A)=\mathbf{P}(A) .
$$

Lema 5 Sejam $\mathbf{Q}_{1}$ e $\mathbf{Q}_{2}$ medidas de probabilidade tais que $\mathbf{Q}_{\mathbf{i}} \ll \mathbf{P}$ para $i=$ $1,2 \ldots$, com $\mathbf{P}$ medida de probabilidade. Então,

$$
\left\|\mathrm{Q}_{2}-\mathrm{Q}_{1}\right\|=\int_{\Omega}\left|\frac{d \mathrm{Q}_{2}}{d \mathrm{P}}(x)-\frac{d \mathrm{Q}_{1}}{d \mathrm{P}}(x)\right| d \mathrm{P}(x) .
$$


Prova. Seja $\Delta \in \Pi$ uma partição qualquer, $\Delta=A_{1}, \ldots, A_{m}$. Então,

$$
\begin{aligned}
\sum_{i=1}^{m}\left|\mathbf{Q}_{2}\left(A_{i}\right)-\mathbf{Q}_{1}\left(A_{i}\right)\right| & =\sum_{i=1}^{m}\left|\int_{A_{i}}\left(\frac{d \mathbf{Q}_{2}}{d \mathbf{P}}(x)-\frac{d \mathbf{Q}_{1}}{d \mathbf{P}}(x)\right) d \mathbf{P}(x)\right| \\
& \leq \sum_{i=1}^{m} \int_{A_{i}}\left|\frac{d \mathbf{Q}_{2}}{d \mathbf{P}}(x)-\frac{d \mathbf{Q}_{1}}{d \mathbf{P}}(x)\right| d \mathbf{P}(x) \\
& =\int_{\Omega}\left|\frac{d \mathbf{Q}_{2}}{d \mathbf{P}}(x)-\frac{d \mathbf{Q}_{1}}{d \mathbf{P}}(x)\right| d \mathbf{P}(x)
\end{aligned}
$$

$\log$,

$$
\left\|\mathrm{Q}_{2}-\mathrm{Q}_{1}\right\| \leq \int_{\Omega}\left|\frac{d \mathbf{Q}_{2}}{d \mathbf{P}}(x)-\frac{d \mathbf{Q}_{1}}{d \mathbf{P}}(x)\right| d \mathbf{P}(x)
$$

Resta agora mostrar o outro lado da desigualdade. Defina

$$
B=\left\{x \in \Omega: \frac{d \mathbf{Q}_{2}}{d \mathbf{P}}(x) \geq \frac{d \mathbf{Q}_{1}}{d \mathbf{P}}(x)\right\} .
$$

Note que $\left\{B, B^{C}\right\}$ é uma partição de $\Omega$, então é verdade que:

$$
\begin{aligned}
\int_{\Omega}\left|\frac{d \mathbf{Q}_{2}}{d \mathbf{P}}(x)-\frac{d \mathbf{Q}_{1}}{d \mathbf{P}}(x)\right| d \mathbf{P}(x) & =\int_{B}\left(\frac{d \mathbf{Q}_{2}}{d \mathbf{P}}(x)-\frac{d \mathbf{Q}_{\mathbf{1}}}{d \mathbf{P}}(x)\right) d \mathbf{P}(x) \\
& +\int_{B^{C}}\left(\frac{d \mathbf{Q}_{1}}{d \mathbf{P}}(x)-\frac{d \mathbf{Q}_{2}}{d \mathbf{P}}(x)\right) d \mathbf{P}(x) \\
& =\mathbf{Q}_{2}(B)-\mathbf{Q}_{\mathbf{1}}(B)+\mathbf{Q}_{1}\left(B^{C}\right)-\mathbf{Q}_{2}\left(B^{C}\right) \\
& \leq \sup _{\Delta \in \Pi} \sum_{i=1}^{m}\left|\mathbf{Q}_{2}\left(A_{i}\right)-\mathbf{Q}_{\mathbf{1}}\left(A_{i}\right)\right| \\
& =\left\|\mathbf{Q}_{2}-\mathbf{Q}_{\mathbf{1}}\right\|
\end{aligned}
$$

Demonstraremos agora a desigualdade de Csiszár:

Teorema 14 Valem as seguintes afirmações:

1. Sejam $\mathbf{P}$ e $\mathbf{Q}$ medidas de probabilidade. Então,

$$
\|\mathbf{P}-\mathbf{Q}\| \leq \sqrt{2 D(\mathbf{P}, \mathbf{Q})}
$$

2. Se $\mathbf{P}_{\mathbf{n}} \rightarrow^{\varepsilon} \mathbf{P} \Rightarrow \lim _{n}\left\|\mathbf{P}_{\mathbf{n}}-\mathbf{P}\right\|=0$

O teorema acima nos dá uma indicação da razão pela qual podemos entender a entropia relativa como uma métrica entre medidas de probabilidade. De fato, se duas medidas estão próximas num sentido entrópico, então, pela desigualdade (1.57), as mesmas duas deverão estar próximas com relação à variação total entre elas, a qual é, de fato, uma distância. 
Prova. (1)Dadas as medidas de probabilidade $\mathbf{P}$ e $\mathbf{Q}$ existe uma terceira medida de probabilidade $\mathbf{S}$ tal que $\mathbf{P} \ll \mathrm{S}$ e $\mathrm{Q} \ll \mathrm{S}$ (por exemplo, tomemos $\left.\mathbf{S}=\frac{1}{2} \mathbf{P}+\frac{1}{2} \mathbf{Q}\right)$, e defina:

$$
B=\left\{x \in \Omega: \frac{d \mathbf{Q}}{d \mathbf{S}}(x) \geq \frac{d \mathbf{P}}{d \mathbf{S}}(x)\right\}
$$

então,

$$
\begin{aligned}
\|\mathbf{P}-\mathbf{Q}\| & =\int_{\Omega}\left|\frac{d \mathbf{P}}{d \mathbf{S}}(x)-\frac{d \mathbf{Q}}{d \mathbf{S}}(x)\right| d \mathbf{S}(x) \\
& =\int_{B}\left(\frac{d \mathbf{Q}}{d \mathbf{S}}(x)-\frac{d \mathbf{P}}{d \mathbf{S}}(x)\right) d \mathbf{S}(x)+\int_{B^{C}}\left(\frac{d \mathbf{P}}{d \mathbf{S}}(x)-\frac{d \mathbf{Q}}{d \mathbf{S}}(x)\right) d \mathbf{S}(x) \\
& =\mathbf{Q}(B)-\mathbf{P}(B)+\mathbf{P}\left(B^{C}\right)-\mathbf{Q}\left(B^{C}\right)
\end{aligned}
$$

Como $\mathrm{P}(B)+\mathrm{P}\left(B^{C}\right)=\mathrm{Q}(B)+\mathrm{Q}\left(B^{C}\right)=1$ temos que

$$
\|\mathbf{P}-\mathbf{Q}\|=2(\mathbf{Q}(B)-\mathbf{P}(B)) .
$$

Ora, $\left\{B, B^{C}\right\}$ é uma partição de $\Omega$, logo pelo lema 4 temos a desigualdade:

$$
D(\mathbf{P}, \mathbf{Q}) \geq \mathbf{Q}(B) \log \frac{\mathbf{Q}(B)}{\mathbf{P}(B)}+\mathbf{Q}\left(B^{C}\right) \log \frac{\mathbf{Q}\left(B^{C}\right)}{\mathbf{P}\left(B^{C}\right)}
$$

Utilizando a seguinte desigualdade: $\forall s>0$ e $\forall t<1$

$$
s \log \frac{s}{t}+(1-s) \log \frac{1-s}{1-t} \geq 2(s-t)^{2}
$$

temos:

$$
\begin{aligned}
D(\mathbf{P}, \mathbf{Q}) & \geq 2(\mathbf{Q}(B)-\mathbf{P}(B))^{2} \\
& =2 \frac{1}{4}\|\mathbf{P}-\mathbf{Q}\|^{2} \\
& =\frac{1}{2}\|\mathbf{P}-\mathbf{Q}\|^{2}
\end{aligned}
$$

e, portanto,

$$
\|\mathbf{P}-\mathrm{Q}\| \leq \sqrt{2 D(\mathbf{P}, \mathbf{Q})}
$$

o que demonstra a primeira parte do teorema. A segunda parte é consequência imediata da primeira.

O próximo teorema mostra qua a entropia relativa satisfaz a propriedade de semi-continuidade inferior. 
Teorema 15 Sejam $\left(\mathbf{P}_{n}\right)$ e $\left(\mathbf{Q}_{n}\right)$ duas sequências de medidas de probabilidade convergindo para $\mathbf{P}$ e $\mathbf{Q}$ no sentido de que dado qualquer conjunto Borelmensurável $A$ tem-se $\lim _{n \rightarrow \infty} \mathbf{P}_{n}(A)=\mathbf{P}(A)$ e $\lim _{n \rightarrow \infty} \mathbf{Q}_{n}(A)=\mathbf{Q}(A)$. Então,

$$
D(\mathbf{P}, \mathbf{Q}) \leq \liminf _{n \rightarrow \infty} D\left(\mathbf{P}_{n}, \mathbf{Q}_{n}\right) \text {. }
$$

Em particular, se $\left(\mathbf{P}_{n}\right)$ e $\left(\mathbf{Q}_{n}\right)$ convergem para $\mathbf{P}$ e $\mathbf{Q}$ em variação, então (1.59) também vale.

Prova. A princípio vamos assumir que $D(\mathbf{P}, \mathbf{Q})<\infty$. Então, pelo lema 4 é verdade que para qualquer $\varepsilon>0$ existe uma partição $\Delta=\left\{A_{1}, \ldots, A_{m}\right\}$ tal que

$$
D_{\Delta}(\mathbf{P}, \mathbf{Q})>D(\mathbf{P}, \mathbf{Q})-\varepsilon
$$

onde $D_{\Delta}(\mathbf{P}, \mathbf{Q})$ é a entropia relativa de $\mathbf{P}$ e $\mathbf{Q}$ sobre a partição $\Delta$. Por hipótese, para cada $A_{i}$ temos

$$
\begin{aligned}
& \lim _{n \rightarrow \infty} \mathbf{P}_{n}\left(A_{i}\right)=\mathbf{P}\left(A_{i}\right) \\
& \lim _{n \rightarrow \infty} \mathbf{Q}_{n}\left(A_{i}\right)=\mathbf{Q}\left(A_{i}\right) .
\end{aligned}
$$

$\mathrm{e}$

Consequentemente, para $n$ suficientemente grande

$$
\left|D_{\Delta}\left(\mathbf{P}_{n}, \mathbf{Q}_{n}\right)-D_{\Delta}(\mathbf{P}, \mathbf{Q})\right| \leq \sum_{i=1}^{m}\left|\mathbf{Q}_{n}\left(A_{i}\right) \log \frac{\mathbf{Q}_{n}\left(A_{i}\right)}{\mathbf{P}_{n}\left(A_{i}\right)}-\mathbf{Q}\left(A_{i}\right) \log \frac{\mathbf{Q}\left(A_{i}\right)}{\mathbf{P}\left(A_{i}\right)}\right|<\varepsilon
$$

$\log \mathrm{O}$

$$
D\left(\mathbf{P}_{n}, \mathbf{Q}_{n}\right) \geq D_{\Delta}\left(\mathbf{P}_{n}, \mathbf{Q}_{n}\right) \geq D_{\Delta}\left(\mathbf{P}_{n}, \mathbf{Q}_{n}\right)-\varepsilon>D(\mathbf{P}, \mathbf{Q})-2 \varepsilon .
$$

Ou seja,

$$
\liminf _{n \rightarrow \infty} D\left(\mathbf{P}_{n}, \mathbf{Q}_{n}\right) \geq D(\mathbf{P}, \mathbf{Q})-2 \varepsilon .
$$

E como $\varepsilon>0$ é arbitrário, segue a desigualdade em (1.59).

Se $D(\mathbf{P}, \mathbf{Q})=\infty$, então, a desigualdade em (1.60) é dada por

$$
D_{\Delta}(\mathbf{P}, \mathbf{Q}) \geq M
$$

onde $M$ é um número positivo arbitrário. Então, seguindo os mesmos passos do argumento apresentado acima temos que

$$
\liminf _{n \rightarrow \infty} D\left(\mathbf{P}_{n}, \mathbf{Q}_{n}\right)=\infty=D(\mathbf{P}, \mathbf{Q}) .
$$




\subsubsection{Representação Dual da Entropia Relativa}

O teorema que enunciaremos agora é uma caracterização funcional da entropia relativa:

Teorema 16 Representação Dual da Entropia Relativa. Dadas duas medidas quaisquer de probabilidade $\mathbf{P}$ e $\mathbf{Q}$ sobre $(\Omega, \mathcal{B}(\Omega))$, tem-se:

$$
D(\mathbf{P}, \mathbf{Q})=\sup _{\Phi \in L^{\infty}(\mathbf{P})}\left[\int_{\Omega} \Phi(x) d \mathbf{Q}(x)-\log \int_{\Omega} \exp (\Phi(x)) d \mathbf{P}(x)\right]
$$

Antes de provarmos o teorema façamos algumas definições e observações que nos auxiliarão mais adiante na demonstração:

$$
\begin{aligned}
\mathcal{U} & :=\mathcal{U}(\mathbf{P})=\left\{u \in L^{1}(\mathbf{P}): u(x) \geq 0 \text { P-q.s. e } \int_{\Omega} u(x) d \mathbf{P}(x)=1\right\} \\
\mathcal{V} & :=\mathcal{V}(\mathbf{P})=\left\{u \in \mathcal{U}: \log u \in L^{\infty}(\mathbf{P})\right\} \\
D_{\mathcal{U}}(\mathbf{P}, \mathbf{Q}) & :=\sup _{u \in \mathcal{U}} \int_{\Omega} \log u(x) d \mathbf{Q}(x) \\
D_{\mathcal{V}}(\mathbf{P}, \mathrm{Q}) & :=\sup _{u \in \mathcal{V}} \int_{\Omega} \log u(x) d \mathbf{Q}(x)
\end{aligned}
$$

e, finalmente, denotemos a função objetiva do lado direito da igualdade (1.61) por $F(\mathbf{P}, \mathbf{Q})$.

obs. 4

para todo $\Phi \in L^{\infty}(\mathbf{P})$ é possível associar uma função:

$$
u(x):=\left[\int_{\Omega} \exp (\Phi(x)) d \mathbf{P}(x)\right]^{-1} \exp (\Phi(x))
$$

que pertence à classe de funções $\mathcal{V}$.

De fato, é evidente que $u \in L^{1}(\mathbf{P}), u \geq 0$ e que $\int_{\Omega} u d \mathbf{P}=1$, portanto, $u \in \mathcal{U}$. Além disso,

$$
\log u(x)=\log (k \Phi(x)) \in L^{\infty}(\mathbf{P})
$$

onde

$$
k=\left[\int_{\Omega} \exp (\Phi(x)) d \mathbf{P}(x)\right]^{-1}
$$

ou seja, $u \in \mathcal{V}$. Mais ainda, vale a relação:

$$
\int_{\Omega} \log u(x) d \mathbf{Q}(x)=\int_{\Omega} \Phi(x) d \mathbf{Q}(x)-\log \int_{\Omega} \exp (\Phi(x)) d \mathbf{P}(x)
$$

e, consequentemente, é verdade que:

$$
F(\mathrm{P}, \mathrm{Q})=D_{\mathcal{V}}(\mathrm{P}, \mathrm{Q})
$$


de modo que, de agora em diante, denotaremos $D_{\mathcal{V}}(\mathbf{P}, \mathbf{Q})$ simplesmente por $F(\mathbf{P}, \mathrm{Q})$.

Tendo terminado estas considerações, podemos demonstrar efetivamente o teorema acima:

Prova. Afirmamos que se $D_{\mathcal{U}}(\mathrm{P}, \mathrm{Q})<\infty$, então, $D(\mathbf{P}, \mathbf{Q}) \leq D_{\mathcal{U}}(\mathrm{P}, \mathrm{Q})$.

Sob a hipótese acima temos que $\mathbf{Q} \ll \mathbf{P}$. Para mostrarmos isso, suponhamos que $\mathbf{P}(A)=0$ para algum $A \in \mathcal{B}(\Omega)$, e para algum $k>0$ escolhido arbitrariamente, definamos

$$
\Phi(x)=k \cdot \chi_{A}(x)
$$

Da fórmula (1.63), temos que

$$
\int_{\Omega} \log u(x) d \mathbf{Q}(x)=\int_{\Omega} \Phi(x) d \mathbf{Q}(x)-\log \int_{\Omega} \exp (\Phi(x)) d \mathbf{P}(x)
$$

e, portanto,

$$
\begin{aligned}
& \int_{\Omega} \log u(x) d \mathbf{Q}(x)=k \mathbf{Q}(A)-\log \left(\int_{\Omega \backslash A} d \mathbf{P}(x)+\int_{A} \exp (k) d \mathbf{P}(x)\right) \\
\Rightarrow & \int_{\Omega} \log u(x) d \mathbf{Q}(x)=k \mathbf{Q}(A)-\log (\mathbf{P}(\Omega \backslash A)+\exp (k) P(A)) \\
\Rightarrow & \int_{\Omega} \log u(x) d \mathbf{Q}(x)=k \mathbf{Q}(A)-\log (\mathbf{P}(\Omega \backslash A)) \\
\Rightarrow & \int_{\Omega} \log u(x) d \mathbf{Q}(x)=k \mathbf{Q}(A)
\end{aligned}
$$

$\log$,

$$
k \mathbf{Q}(A)=\int_{\Omega} \log u(x) d \mathbf{Q}(x) \leq D_{\mathcal{U}}(\mathbf{P}, \mathbf{Q})<\infty
$$

e como $k$ foi escolhido arbitrariamente segue que $\mathbf{Q}(A)=0$, ou seja, $\mathbf{Q} \ll \mathbf{P}$. Ora, então existe a derivada de Radón-Nikodin $\frac{d \mathbf{Q}}{d \mathrm{P}}$ e, além disso, $\frac{d \mathbf{Q}}{d \mathbf{P}} \in \mathcal{U}$, portanto,

$$
D(\mathbf{P}, \mathbf{Q})=\int_{\Omega} \frac{d \mathbf{Q}}{d \mathbf{P}}(x) d \mathbf{Q}(x) \leq D_{\mathcal{U}}(\mathbf{P}, \mathbf{Q})
$$

e isto demonstra a afirmação.

Afirmamos agora que se $F(\mathbf{P}, \mathbf{Q})<\infty$ então $D_{\mathcal{U}}(\mathbf{P}, \mathbf{Q}) \leq F(\mathbf{P}, \mathbf{Q})$. Notemos que é suficiente mostrar que $\int_{\Omega} \log u(x) d \mathbf{Q}(x) \leq F(\mathbf{P}, \mathbf{Q})$ para todo $u \in \mathcal{U}$.

Seja, portanto, $u \in \mathcal{U}$ qualquer. Como estamos assumindo que $F(\mathbf{P}, \mathbf{Q})<\infty$ então também temos que $(\log u(x))^{-} \equiv-\min (\log u(x), 0) \in L^{1}(\mathbf{Q})$. Seja

$$
u_{n}(x):=\left(u(x) \vee \frac{1}{n}\right) \wedge n
$$

para $n=1,2, \ldots$ e defina $\Phi_{n}(x) \equiv \log u_{n}(x)$. Notemos que $\Phi_{n} \in L^{\infty}(\mathbf{P})$ e que pela fórmula (1.63)

$$
\int_{\Omega} \Phi_{n}(x) d \mathbf{Q}(x) \leq \log \int_{\Omega} \exp \left(\Phi_{n}(x)\right) d \mathbf{P}(x)+F(\mathbf{P}, \mathbf{Q})
$$


Como $0 \leq u_{n}(x)=\exp \left(\Phi_{n}(x) \leq u(x)+1\right)$ segue do teorema da convergência dominada de Lebesgue que

$$
\lim _{n \rightarrow \infty} \int_{\Omega} \exp \left(\Phi_{n}(x)\right) d \mathbf{P}(x)=\int_{\Omega} u(x) d \mathbf{P}(x)=1
$$

Notemos ainda que $0 \leq\left(\Phi_{n}(x)\right)^{+} \leq\left(\Phi_{n+1}(x)\right)^{+} \leq(\log u(x))^{+}$e que $0 \leq$ $\left(\Phi_{n}(x)\right)^{-} \leq\left(\Phi_{n+1}(x)\right)^{-} \leq(\log u(x))^{-}$, logo, pelo teorema da convergência monótona

$$
\lim _{n \rightarrow \infty} \int_{\Omega} \Phi_{n}(x) d \mathbf{P}(x)=\int_{\Omega} \lim _{n \rightarrow \infty} \Phi_{n}(x) d \mathbf{P}(x)=\int_{\Omega} \log u(x) d \mathbf{P}(x) .
$$

Passando ao limite a fórmula (1.66) e aplicando os resultados obtidos acima, temos:

$$
\int_{\Omega} \log u(x) d \mathbf{P}(x) \leq \log 1+F(\mathbf{P}, \mathbf{Q})
$$

ou seja,

$$
\int_{\Omega} \log u(x) d \mathbf{P}(x) \leq F(\mathbf{P}, \mathbf{Q})
$$

para todo $u \in \mathcal{U}$. Logo,

$$
D_{\mathcal{U}}(\mathbf{P}, \mathbf{Q}) \leq F(\mathbf{P}, \mathbf{Q})
$$

Enfim, resta-nos apenas mostrar que, se $D(\mathbf{P}, \mathbf{Q})<\infty$, então:

$$
F(\mathbf{P}, \mathrm{Q}) \leq D(\mathbf{P}, \mathbf{Q})
$$

Como estamos assumindo que $D(\mathbf{P}, \mathbf{Q})<\infty$ temos $\mathbf{P} \ll \mathbf{Q}$. Dado $u \in \mathcal{V}$, defina $d \mathbf{G}(x):=u(x) d \mathbf{P}(x)$. Então, $\mathbf{Q} \ll \mathbf{P} \ll \mathbf{G}$, pois, $u(x) \geq 0 \mathbf{P}-q . s$.. Logo,

$$
\begin{aligned}
0 \leq D(\mathrm{G}, \mathrm{Q}) & =\int_{\Omega} \log \left(\frac{d \mathbf{Q}}{d \mathbf{G}}(x)\right) d \mathbf{Q}(x) \\
& =\int_{\Omega} \log \left(\frac{d \mathbf{Q}}{d \mathbf{P}}(x) \frac{d \mathbf{P}}{d \mathbf{G}}(x)\right) d \mathbf{Q}(x) \\
& =\int_{\Omega} \log \left(\frac{d \mathbf{Q}}{d \mathbf{P}}(x)\right) d \mathbf{Q}(x)+\int_{\Omega} \log \left(\frac{d \mathbf{P}}{d \mathbf{G}}(x)\right) d \mathbf{Q}(x) \\
& =D(\mathbf{P}, \mathbf{Q})-\int_{\Omega} \log u(x) d \mathbf{Q}(x)
\end{aligned}
$$

e, portanto,

$$
\Rightarrow D(\mathbf{P}, \mathbf{Q}) \geq \int_{\Omega} \log u(x) d \mathbf{Q}(x) .
$$

Ora, como a função $u$ foi escolhida arbitrariamente em $\mathcal{V}$ segue a seguinte desigualdade:

$$
F(\mathrm{P}, \mathrm{Q}) \leq D(\mathrm{P}, \mathrm{Q}) .
$$


Temos, portanto, que a partir das desigualdades (1.65), (1.67) e (1.69) vale a relação:

$$
D(\mathbf{P}, \mathbf{Q})=F(\mathbf{P}, \mathbf{Q})
$$

ou seja,

$$
D(\mathbf{P}, \mathbf{Q})=\sup _{\Phi \in L^{\infty}(\mathbf{P})}\left[\int_{\Omega} \Phi(x) d \mathbf{Q}(x)-\log \int_{\Omega} \exp (\Phi(x)) d \mathbf{P}(x)\right]
$$

como queríamos demonstrar.

\subsubsection{Caracterização Financeira da Entropia}

Assumamos que nosso espaço de estados seja dado por $(\Omega, \mathcal{B}, \mathbf{P})$ onde $\Omega$ representa os possíveis estados da natureza (trajetórias dos preços) e $\mathbf{P}$ representa a probabilidade natural da economia, ou seja, ela é a melhor medida existente (entre todas as medidas de probabilidade), e a denominaremos de medida real da economia ou mercado.

Definição 9 Sejam u uma função utilidade arbitrária e $X \in L^{\infty}$ representando um payoff qualquer ou o consumo (de fato, qualquer variável econômica cuja medida de utilidade faça sentido). Chamamos Equivalente Certo o payoff $X$ associado a função de utilidade u a funçao $c_{u}(X)$ definida por:

$$
c_{u}(X)=u^{-1}\left(\mathbf{E}_{\mathbf{P}}[u(X)]\right)
$$

Sua interpretação é imediata. Por exemplo, se $X$ for um fluxo de caixa incerto, então, $c_{u}(X)$ representa o nível de capital em risco para o qual a utilidade de um agente hoje iguala a utilidade do valor esperado da utilidade do retorno esperado amanhã. Ou seja, $c_{u}(X)$ serve de parâmetro na tomada de decisões quando estas baseiam-se na utilidade. Ou seja, determinando o equivalente certo de um agente é como se estivessemos escolhendo um Var aceitável para esse agente. Note também que se $c_{u}(X)$ é expresso nas mesmas unidades de $X$, portanto, se $X$ tem algum significado monetário o mesmo acontece com o equivalente certo.

O próximo resultado relaciona Equivalentes Certos com a entropia relativa. Mais ainda ele dá preços para a entropia relativa e avalia o custo pela escolha de uma medida de probabilidade que não seja a medida real, isto é, que não seja a "melhor" dentre todas as possibilidades. Desse modo a entropia relativa nada mais é do que uma penalização devido a má escolha da medida de probabilidade uma vez que essa não representa idealmente a informação disponível. Mais ainda, o próximo resultado fornece à entropia relativa significado monetário no sentido de que a mesma pode passar a ser expressa em unidades monetárias. Ou seja, a entropia deixa de ser meramente um conceito teórico e passa a ter um significado mais concreto em termos de dinheiro. 
Lema 6 Dada a função de utilidade exponencial

$$
u_{\alpha}(x)=-\frac{1}{\alpha} e^{-\alpha x}
$$

com $\alpha>0$, então para todo $X \in L^{\infty}$ e para toda constante $K$, vale:

$$
c_{u}(X+K)=c_{u}(X)+K .
$$

Prova. Seja $X \in L^{\infty}$. Em primeiro lugar note que $u_{\alpha}^{-1}(X)=-\frac{1}{\alpha} \log (-\alpha X)$ e, portanto, podemos calcular explicitamente o equivalente certo:

$$
c_{u}(X)=-\frac{1}{\alpha} \log \left(\mathbf{E}^{\mathrm{P}}\left[e^{-\alpha X}\right]\right) .
$$

Então,

$$
\begin{aligned}
c_{u}(X+K) & =-\frac{1}{\alpha} \log \left(\mathbf{E}^{\mathbf{P}}\left[e^{-\alpha(X+K)}\right]\right)=-\frac{1}{\alpha} \log \left(e^{-\alpha K} \mathbf{E}^{\mathbf{P}}\left[e^{-\alpha X}\right]\right) \\
& =K+c_{u}(X) .
\end{aligned}
$$

Proposição 4 (Entropia como $\mathrm{VaR}^{2}$ ) Seguindo a mesma notação do lema acima, temos:

$$
\frac{1}{\alpha} D(\mathbf{P}, \mathbf{Q})=\sup _{X \in L^{\infty}: \mathbf{E}_{\mathbf{Q}}[X] \leq 0} c_{u_{\alpha}}(X) .
$$

Prova.As medidas $\mathbf{P}$ e $\mathbf{Q}$ são dadas e, de agora em diante, assumiremos que dado qualquer payoff $X \in L^{\infty}(\mathrm{P})$, ele também é um elemento de $L^{\infty}(\mathrm{Q})$.

Afirmemos que:

$$
\sup \left\{c_{u_{\alpha}}(X): \mathbf{E}^{\mathbf{Q}}[X] \leq 0\right\}=\sup \left\{c_{u_{\alpha}}(X): \mathbf{E}^{\mathbf{Q}}[X]=0\right\}
$$

Como $\left\{c_{u_{\alpha}}(X): \mathbf{E}^{\mathbf{Q}}[X] \leq 0\right\} \supseteq\left\{c_{u_{\alpha}}(X): \mathbf{E}^{\mathbf{Q}}[X]=0\right\}$, então,

$$
\sup \left\{c_{u_{\alpha}}(X): \mathbf{E}^{\mathbf{Q}}[X] \leq 0\right\} \geq \sup \left\{c_{u_{\alpha}}(X): \mathbf{E}^{\mathbf{Q}}[X]=0\right\} .
$$

Por outro lado, pelo lema acima,

$$
c_{u_{\alpha}}(X) \leq c_{u_{\alpha}}(X)-\mathbf{E}^{\mathbf{Q}}[X]=c_{u_{\alpha}}\left(X-\mathbf{E}^{\mathbf{Q}}[X]\right),
$$

e como $\mathrm{E}^{\mathbf{Q}}\left[X-\mathrm{E}^{\mathbf{Q}}[X]\right]=0$ segue que:

$$
c_{u_{\alpha}}\left(X-\mathbf{E}^{\mathbf{Q}}[X]\right) \leq \sup \left\{c_{u_{\alpha}}(Z): Z \in L^{\infty}(\mathrm{Q}), \mathbf{E}^{\mathbf{Q}}[Z]=0\right\} .
$$

e, uma vez que $X$ foi tomado arbitrariamente, temos:

$$
\sup \left\{c_{u_{\alpha}}(X): \mathbf{E}^{\mathbf{Q}}[X] \leq 0\right\} \leq \sup \left\{c_{u_{\alpha}}(X): \mathbf{E}^{\mathbf{Q}}[X]=0\right\}
$$

\footnotetext{
${ }^{2}$ VaR: Value at Risk
} 
Portanto de (1.75) e (1.76) segue a afirmação (1.74).

Afirmamos agora que:

$$
\sup \left\{c_{u_{\alpha}}(X): \mathbf{E}^{\mathbf{Q}}[X]=0\right\}=\sup \left\{c_{u_{\alpha}}(X)-\mathbf{E}^{\mathbf{Q}}[X]: X \in L^{\infty}(\mathbf{Q})\right\}
$$

Ora, mas pelo lema acima $c_{u_{\alpha}}(X)-\mathbf{E}^{\mathbf{Q}}[X]=c_{u_{\alpha}}\left(X-\mathbf{E}^{\mathbf{Q}}[X]\right)$, e como o valor esperado de $X-\mathbf{E}^{\mathbf{Q}}[X]$ segundo a medida $\mathbf{Q}$ é nulo, segue que

$$
c_{u_{\alpha}}(X)-\mathbf{E}^{\mathbf{Q}}[X] \in\left\{c_{u_{\alpha}}(X): \mathbf{E}^{\mathbf{Q}}[X]=0\right\}
$$

e, portanto

$$
\left\{c_{u_{\alpha}}(X)-\mathbf{E}^{\mathbf{Q}}[X]: X \in L^{\infty}(\mathbf{Q})\right\} \subset\left\{c_{u_{\alpha}}(X): \mathbf{E}^{\mathbf{Q}}[X]=0\right\} .
$$

Consequentemente,

$$
\sup \left\{c_{u_{\alpha}}(X)-\mathbf{E}^{\mathrm{Q}}[X]: X \in L^{\infty}(\mathbf{Q})\right\} \leq \sup \left\{c_{u_{\alpha}}(X): \mathbf{E}^{\mathbf{Q}}[X]=0\right\} .
$$

Por outro lado, seja $X$ tal que $\mathbf{E}^{\mathbf{Q}}[X]=0$. Então,

$$
\begin{aligned}
c_{u_{\alpha}}(X) & =c_{u_{\alpha}}(X)-\mathbf{E}^{\mathrm{Q}}[X]=c_{u_{\alpha}}\left(X-\mathbf{E}^{\mathrm{Q}}[X]\right) \\
& \leq \sup \left\{c_{u_{\alpha}}(X)-\mathbf{E}^{\mathrm{Q}}[X]: X \in L^{\infty}(\mathbf{Q})\right\}
\end{aligned}
$$

e como $X$ foi escolhido arbitrariamente, segue que

$$
\sup \left\{c_{u_{\alpha}}(X): \mathbf{E}^{\mathrm{Q}}[X]=0\right\} \leq \sup \left\{c_{u_{\alpha}}(X)-\mathbf{E}^{\mathrm{Q}}[X]: X \in L^{\infty}(\mathbf{Q})\right\} .
$$

Logo, de (1.78) e (1.79) segue a afirmação (1.77).

Então, das igualdades (1.74) e (1.77) temos:

$$
\begin{aligned}
\sup \left\{c_{u_{\alpha}}(X): \mathbf{E}^{\mathbf{Q}}[X] \leq 0\right\} & =\sup \left\{c_{u_{\alpha}}(X)-\mathbf{E}^{\mathbf{Q}}[X]: X \in L^{\infty}(\mathbf{Q})\right\} \\
& =\sup _{X \in L^{\infty}(\mathbf{Q})}\left(-\frac{1}{\alpha} \log \mathbf{E}^{\mathbf{P}}\left[e^{-\alpha X}\right]-\mathbf{E}^{\mathbf{Q}}[X]\right)
\end{aligned}
$$

e efetuando a mudança de variáveis: $Z=-\alpha X$ obtemos:

$$
\begin{aligned}
\sup _{X \in L^{\infty}(\mathbf{Q}): \mathbf{E}^{\mathbf{Q}}[X] \leq 0} c_{u_{\alpha}}(X) & =\sup _{Z \in L^{\infty}(\mathbf{Q})}\left(-\frac{1}{\alpha} \log \mathbf{E}^{\mathbf{P}}\left[e^{Z}\right]+\frac{1}{\alpha} \mathbf{E}^{\mathbf{Q}}[Z]\right) \\
& =\frac{1}{\alpha} \sup _{Z \in L^{\infty}(\mathbf{Q})}\left(-\log \mathbf{E}^{\mathbf{P}}\left[e^{Z}\right]+\mathbf{E}^{\mathbf{Q}}[Z]\right) \\
& =\frac{1}{\alpha} D(\mathbf{P}, \mathbf{Q})
\end{aligned}
$$

pela igualdade em (1.61). 


\section{Capítulo 2}

\section{Teoria Entrópica de Apreçamento}

\subsection{Introdução}

Nesse capítulo introduziremos a teoria da informação dentro do contexto da teoria de apreçamento de ativos em mercados incompletos como um método capaz de determinar a medida de Arrow-Debreu que o mercado (agentes) deve usar ao apreçar quaisquer ativos. Lembremos que em mercados incompletos existem inúmeras medidas de Arrow-Debreu e todas elas são consistentes em relação ao Princípio da Não-Arbitragem e, portanto, a escolha de qualquer uma delas por parte dos agentes deve ser arbitrária. O que fazemos nessa seção é introduzir hipóteses (ou axiomas) na teoria de apreçamento de ativos a qual propicia a unicidade da medida de Arrow-Debreu. Chamaremos tal hipótese de Princípio da Máxima Entropia ou Princípio da Mínima Entropia Relativa as quais são em um certo sentido equivalentes. Começamos o capítulo definindo os conceitos econômicos de modo que a discussão dos conceitos acima seja mais precisa. Em seguida, introduzimos o Princípio da Máxima Entropia e como utilizá-lo no apreçamento de ativos. Nas seções seguintes provamos os resultados necessários para fundamentar a teoria e, finalmente, concluímos sua aplicação explicitando a expressão da única medida de Arrow-Debreu consistente com a nova hipótese. As duas últimas seções são devotadas a mais duas aplicações do Princípio da Máxima Entropia. Em particular, na penúltima seção deduzimos a expressão do Núcleos de Apreçamento que está de acordo com a hipótese recém introduzida e na última seção fazemos uma aplicação aos derivativos mostrando que ao assumir uma medida de referência log-normal o Princípio da Mínima Entropia Relativa induz a mesma medida de Arrow-Debreu obtida a partir do modelo de Black-Scholes-Merton. 


\subsection{Entropia em Mercados Incompletos}

\section{Distribuições Neutras ao Risco em Economias de Um Período com Espaço de Estados Discreto}

Consideremos uma economia de um período (com vencimento numa data futura $T$ ) e cujos estados são discretos e representados pelo conjunto

$$
\{1, \ldots, U\}
$$

isto é, nossa economia deve necessariamente atingir um desses estados no decorrer do tempo. Também suponhamos que existem $N$ ativos nessa economia, cujos preços são dados pelo vetor

$$
\pi:=\left(\begin{array}{c}
\pi_{1} \\
\vdots \\
\pi_{N}
\end{array}\right)
$$

e cujos payoffs são dados pela matriz de payoffs

$$
\mathrm{X}:=\left(\begin{array}{ccc}
X_{11} & \cdots & X_{1 U} \\
\vdots & \ddots & \vdots \\
X_{N 1} & \cdots & X_{N U}
\end{array}\right)
$$

onde $X_{i j}$ representa o payoff do ativo $i$ no estado $j$. Além disso, um investidor deve decidir como alocar seus recursos nesses ativos determinando quantas unidades (ou frações) de cada deseja obter. Matematicamente, expressamos essa alocação através de um vetor

$$
\alpha:=\left(\begin{array}{c}
\alpha_{1} \\
\vdots \\
\alpha_{N}
\end{array}\right) \in \mathbb{R}^{N}
$$

e a denominamos de portfólio. O valor de mercado do portfólio é dado por $\alpha^{T} \pi$, e seu payoff por $\mathrm{X}^{T} \alpha$. Definimos

$$
\Psi:=\left(\begin{array}{c}
\psi_{1} \\
\vdots \\
\psi_{U}
\end{array}\right) \in \mathbb{R}^{S}
$$

então, como o vetor de preços dos estados (ou state-prices vector) se $\pi=\mathrm{X} \Psi$. Notemos que $\Psi$ é solução de um sistema linear e, como tal, pode não admitir nenhuma solução, assim como apenas uma ou até mesmo infinitas soluções. Observemos também que

$$
\pi_{i}=\sum_{j=1}^{U} X_{i j} \psi_{j}
$$


logo, podemos interpretar $\psi_{j}$ como o custo marginal em obter uma unidade a mais do payoff no estado $j$.

Um conceito essencial que devemos definir agora é o da arbitragem:

Definição 10 Uma arbitragem é um portfólio $\alpha \in \mathbb{R}^{N}$ que satisfaz uma das seguintes condições:

1. $\alpha^{T} \pi \leq 0$ e $\mathrm{X}^{T} \alpha>0^{1}$, ou

2. $\alpha^{T} \pi<0$ e $\mathrm{X}^{T} \alpha \geq 0$.

Ou seja, a existência de arbitragem implica que é possível ganhar "algo" sem custo algum. Aqui entra aquela que é talvez a mais importante hipótese dentro da teoria econômico-financeira - o Princípio da Não-Arbitragem - o qual afirma que não existem oportunidades de arbitragem na economia. É sabida a equivalência entre a existência de um vetor de preços dos estados $\Psi$ e o princípio da não-arbitragem.

Teorema $17^{2}$ Não existem oportunidades de arbitragem se, e somente se, existe um vetor de preços dos estados.

Ora, como estamos assumindo o princípio da não-arbitragem, temos garantida a existência de pelo menos um vetor de preços de estados. No entanto, não existe nenhuma garantia quanto a unicidade e, como veremos mais adiante, é nesse ponto que reside a importância da entropia. Contudo, antes de a introduzirmos em nossa discussão, é preciso definir o conceito de probabilidades de ArrowDebreu. Pela fórmula (2.5) podemos observar que normalizando tal equação, dividindo-a por $\hat{\psi}:=\sum_{j=1}^{U} \psi_{j}$ obtemos uma nova distribuição de probabilidades $\hat{\Psi}:=\left(\frac{\psi_{1}}{\hat{\psi}}, \ldots, \frac{\psi_{U}}{\hat{\psi}}\right)$ e a seguinte relação

$$
\frac{\pi_{i}}{\hat{\psi}}=\frac{1}{\hat{\psi}} \sum_{j=1}^{U} X_{i j} \psi_{j}:=\hat{\mathbf{E}}\left[\mathbf{X}_{i}\right]
$$

onde $\mathrm{X}_{i}=\left(X_{i 1}, \ldots, X_{i U}\right)$, ou seja, temos agora que o preço normalizado do $i$ ésimo ativo é o valor esperado do seu payoff segundo essas novas probabilidades.

Suponha que exista um portfólio $\alpha_{0}$ cujo payoff seja $\mathrm{X}^{T} \alpha_{0}=(1, \ldots, 1)^{T}$, isto é, um portfólio ajustado ao risco, então, pelo princípio de não-arbitragem, o preço desse portfólio deve igualar a taxa de desconto livre-de-risco, logo, $\hat{\psi}$ deve

\footnotetext{
${ }^{1} \mathrm{~A}$ notação a $>0$, onde a é um vetor n-dimensional, indica que todas suas coordenadas são estritamente positivas.

${ }^{2}$ Veja [6] para a demonstração do teorema, assim como para uma discussão mais profunda dos conceitos acima.
} 
igualar tal taxa. De fato,

$$
\begin{aligned}
\text { Taxa de Desconto Livre-de-Risco } & =\alpha_{0}^{T} \pi=\sum_{i=1}^{N} \pi_{i} \\
& =\sum_{i=1}^{N} \hat{\psi} \hat{\mathbf{E}}\left[\mathbf{X}_{i}\right]=\hat{\psi} \hat{\mathbf{E}}\left[\sum_{i=1}^{N} \mathbf{X}_{i}\right] \\
& =\hat{\psi} \hat{\mathbf{E}}\left[\left(\sum_{i=1}^{N} X_{i 1} 1, \ldots, \sum_{i=1}^{N} X_{i S} 1\right)^{T}\right] \\
& =\hat{\psi} \hat{\mathbf{E}}\left[\mathbf{X}^{T} \alpha\right] \\
& =\hat{\psi}
\end{aligned}
$$

como queríamos demonstrar. Mais ainda, na existência de um portfólio neutro ao risco, o preço de qualquer ativo $i=1, \ldots, N$ é dado pelo valor descontado pela taxa livre-de-risco da esperança do vetor de payoffs $\mathbf{X}_{i}$, ou seja,

$$
\pi_{i}=\hat{\psi} \hat{\mathbf{E}}\left[\mathbf{X}_{i}\right]
$$

segundo essas novas distribuições de probabilidade. Note que na presença de um ativo neutro ao risco, ou seja, cujo valor do seu payoff independe do estado em que o mundo se encontra, sempre será possível construir aquele portfólio também neutro ao risco, bastando, para isso, compor tal portfólio unicamente com o ativo mencionado.

Reciprocamente, se $\hat{\psi}$ é, por hipótese, a taxa de desconto livre de risco e se existe uma distribuição de probabilidade $\mathbf{p}=\left(p_{1}, \ldots, p_{U}\right)^{T}$ tal que o preço do $i$-ésimo ativo, $\pi_{i}$, é igual ao valor descontado (por $\hat{\psi}$ ) da esperança de $\mathbf{X}_{i}$, então, existe um único vetor de preços dos estados dado por $\hat{\psi} \mathbf{p}$. De fato,

$$
\begin{aligned}
\pi_{i} & =\hat{\psi} \hat{\mathbf{E}}^{\mathrm{p}}\left[\mathbf{X}_{i}\right]=\hat{\psi} \sum_{j=1}^{U} p_{j} X_{i j} \\
& =\sum_{j=1}^{U} \hat{\psi} p_{j} X_{i j}=\mathbf{X}_{i} \hat{\psi} \mathbf{p}
\end{aligned}
$$

ou seja,

$$
\pi=\mathrm{X} \hat{\psi} \mathbf{p}
$$

e, portanto, $\hat{\psi} \mathbf{p}$ é um vetor de preços de estados.

Consequentemente, na hipótese de um ativo livre-de-risco (por exemplo, um título qualquer), para cada vetor de preços de estados temos uma única distribuição de probabilidade tal que o preço do $i$-ésimo ativo iguala-se ao valor esperado (de acordo com essa distribuição) do seu payoff descontado pela taxa livre-de-risco. Denominamos tais distribuições por distribuições de ArrowDebreu. 


\section{Mercados Completos e Incompletos}

Temos, então, duas possibilidades: há unicidade com relação ao vetor de preços dos estados, logo, existe uma única distribuição de Arrow-Debreu e, consequentemente, o preço do ativo está bem-definido. Uma segunda possibilidade seria a não-unicidade do vetor de preços dos estados, implicando dessa maneira a existência de mais de uma distribuição de Arrow-Debreu e, consequentemente, mais de um preço para os ativos de nossa economia (todos eles de acordo com o princípio da não arbitragem). São exatamente esses conceitos que determinam a completude ou incompletude de um mercado, sendo o mercado completo quando existe apenas uma única distribuição de Arrow-Debreu e incompleto caso contrário. A seguir, expressaremos essas noções de forma mais precisa.

Definição 11 Dizemos que um mercado é completo se o espaço gerado por $\mathrm{X}$ é igual a $\mathbb{R}^{U}$, isto é, se $\left\langle\left\{\mathrm{X} \alpha: \alpha \in \mathbb{R}^{N}\right\}\right\rangle=\mathbb{R}^{U}$. Caso contrário, o mercado é dito incompleto.

Em outras palavras, o mercado é completo se qualquer vetor de payoffs prédeterminado pode ser atingido com a escolha correta do portfólio. Evidentemente, se o mercado é completo, de acordo com a definição acima, então as $U$ colunas da matriz de payoffs são linearmente independentes e, portanto, tal matriz é invertível. Logo, $\pi=\mathrm{X} \Psi$ implica que $\Psi=\mathrm{X}^{-1} \pi$ e, consequentemente, existe uma única distribuição de Arrow-Debreu.

\section{Introduzindo o Princípio da Máxima Entropia}

A pergunta natural que surge é como escolher, dentre todas possibilidades, a "melhor" distribuição de Arrow-Debreu no caso de mercados incompletos. Como estudamos nos primeiro e segundo capítulos, distribuições com maior entropia carregam menos informação, a idéia aqui é escolher aquela distribuição, $\pi^{*}$, que traduz exatamente a informação conhecida pelos agentes do mercado. Nós incorporamos tais informações através de restrições em um programa de otimização da forma:

$$
\pi^{*}=\operatorname{argmax}_{\pi} h(\pi)
$$

sujeito a um conjunto de restrições, ou conjunto informacional, I. Caso uma outra distribuição neutra ao risco seja escolhida, distorções estarão sendo introduzidas, pois essa distribuição traz embutidas informações não conhecidas a priori e, eventualmente, atribui mais probabilidade a eventos menos prováveis e vice-versa. Lembremos que a distribuição que maximiza a entropia é, na verdade, aquela que está, informacionalmente, mais próxima da distribuição uniforme, ilustrando que houve a exata translação do estado de máxima ignorância para um novo estado onde algumas novas informações foram introduzidas. Se, a priori (via algum teste econométrico, por exemplo) fosse sabido que, uma determinada distribução (ex-post) modelava o mercado, então através desse enfoque informacional da economia poderíamos incorporar também mais essa informação na determinação da distribuição de Arrow-Debreu ótima, escolhendo aquela que minimiza a "distância" entrópica com relação à distribuição de referência e que, 
além disso, satisfaz as condições impostas pelo conjunto informacional $I$. Podemos resumir a exposição acima através dos princípios, ou axiomas, abaixo:

Princípio da Máxima Entropia. A distribuição de Arrow-Debreu que equlibra o mercado deve maximizar a entropia condicional às informações, ou restrições, contidas em $I$.

Ou através de sua contra-parte:

Princípio da Mínima Entropia Relativa. Conhecida uma distribuição a priori ('prior'), a distribuição de Arrow-Debreu que equilibra o mercado deve minimizar a entropia relativa condicional às informações, ou restrições, contidas em $I$.

Note que pelo Princípio da Mínima Entropia que, intuitivamente falando, o prior é minimamente modificado de modo a obtermos uma medida neutra ao risco.

Vamos considerar agora um caso concreto de inferência da distribuição de Arrow-Debreu realizada pelo investidor. Vamos supor que existem dois ativos, um ativo livre-de-risco, e uma ação representando o ativo de risco. Em primeiro lugar, devemos checar qual é a informação a sua disposição. Nós assumiremos que ele conhece (ou decide quais são) (i) o espaço onde moram suas probabilidades (a princípio assumiremos que elas são discretas e, consequentemente, moram em algum simplex contido em um espaço euclidiano de dimensão finita $U$ - com isso resolvemos o problema quando a modelagem do mercado utiliza árvores multinomiais) - (ii) o preço de um título padrão livre-de-risco, $\pi_{0}$ que no final do período paga uma unidade monetária, (iii) o preço corrente da ação, $S$ e, finalmente, (iv) a volatilidade do preço da ação (estimada a priori, através dos dados disponíveis ao investidor). Obviamente, se $M>2$, estamos numa situação de mercado incompleto e o investidor tem uma variedade de distribuições para escolher. Pela fórmula (2.6), se queremos que a distribuição resultante seja neutra ao risco, devemos ter a seguinte condição satisfeita: $\sum_{i=1}^{U} p_{i} S_{i}=\frac{S}{P}$. Ora, então, pelo princípio da máxima entropia, podemos escrever esse problema na forma do seguinte programa:

$$
\max _{\mathbf{p}}-\sum_{i=1}^{U} p_{i} \log p_{i}
$$

sujeito a:

$$
\begin{cases}\sum_{i=1}^{U} p_{i}=1 & \text { com } p_{i}>0 \\ \sum_{i=1}^{U} p_{i} S_{i}=\frac{S}{P} & \\ \sum_{i=1}^{U} p_{i} S_{i}^{2}=\sigma^{2}+\frac{S^{2}}{P^{2}} & \end{cases}
$$

onde a última restrição representa uma aposta do investidor ou um consenso do mercado com relação à volatilidade do preço da ação. 
Se estivessemos interessados em distribuições contínuas de probabilidade, então o programa de otimização acima seria reescrito da forma

$$
\max _{f}-\int f(s) \log f(s) d s
$$

sujeito a:

$$
\left\{\begin{array}{l}
f \geq 0 \\
\int f(s) d s=1 \\
\int s f(s) d s=\frac{S_{1}}{P} \\
\int s^{2} f(s) d s=\sigma^{2}+\frac{S_{1}^{2}}{P^{2}}
\end{array}\right.
$$

Mais ainda se uma medida de referência fosse conhecida, então o programa de otimização seria, por exemplo, da forma

$$
\min _{f}-\int_{S_{\min }}^{S_{\max }} f(s) \log \frac{f(s)}{u(s)} d s
$$

sujeito a:

$$
\left\{\begin{array}{l}
f \geq 0 \\
\int f(s) d s=1 \\
\int s f(s) d s=\frac{S_{1}}{P} \\
\int s^{2} f(s) d s=\sigma^{2}+\frac{S_{1}^{2}}{P^{2}}
\end{array}\right.
$$

Notemos que no caso discreto temos um problema de otimização em $\mathbb{R}^{n}$ para algum natural $n$. No entanto, no caso contínuo estamos trabalhando em espaços de funções. Esse problema de otimização pode ser ainda mais complicado se considerarmos espaços de medidas (de probabilidade) mais gerais. Notemos também que todos problemas de otimização acima envolvem restrições sobre esperanças. As próximas seções formalizam e tratam essas questões do caso mais simples (distribuições em $\mathbb{R}^{n}$ ) ao mais complicado (medidas de probabilidade gerais).

\subsection{Minimizando a Entropia Relativa}

Nessa seção trataremos de alguns aspectos geométricos da entropia relativa e investigaremos as circunstâncias em que é possível minimizá-la no sentido de que dada uma medida a priori, a qual denominaremos medida de referência, tentarmos encontrar uma distribuição de probabilidade satisfazendo algumas restrições e que ao mesmo tempo seja a mais próxima da medida de referência. Ou seja, temos um problema de existência e unicidade pela frente.

\subsubsection{Casos Discreto e Contínuo}

Em primeiro lugar, começaremos analisando o caso discreto. Já vimos no primeiro capítulo que a entropia relativa é não negativa e que ela anula-se quando 
suas entradas são iguais, portanto, se não impomos nenhuma restrição, então, dada uma medida $\mathbf{P}$ a priori, aquela que minimiza a entropia relativa é a própria P. Consequentemente, passemos a analisar o caso em que restrições são impostas, via multiplicadores de Lagrange. Formalmente, nosso problema é dado por: conhecida uma distribuição de probabilidades $\mathbf{p}=\left(p_{1}, \ldots, p_{n}\right)$, resolver o problema de otimização:

$$
\begin{cases}\min _{\mathrm{q}} D(\mathbf{p}, \mathbf{q})=\sum_{i=1}^{n} q_{i} \log \frac{q_{i}}{p_{i}} & \text { onde } \mathbf{q}=\left(q_{1}, \ldots, q_{n}\right), \text { sujeito a: } \\ \sum_{i=1}^{n} q_{i}=1 ; & \text { para } j=1, \ldots, M . \\ \sum_{i=1}^{n} \zeta_{i j} q_{i}=C_{j} & \end{cases}
$$

onde $\zeta_{i j} \in \mathbb{R}$, para todo $j=1, \ldots, M$. Ora, resolver esse problema equivale a encontrar o $\left(q_{1}, \ldots, q_{n} ; \mu ; \lambda_{1}, \ldots, \lambda_{M}\right)$-ponto-crítico da função

$$
\begin{aligned}
\mathcal{L}\left(q_{1}, \ldots, q_{n} ; \mu ; \lambda_{1}, \ldots, \lambda_{M}\right)= & \sum_{i=1}^{n} q_{i} \log \frac{q_{i}}{p_{i}}+\mu\left(\sum_{i=1}^{n} q_{i}-1\right) \\
& +\sum_{j=1}^{M} \lambda_{j}\left(\sum_{i}^{n} \zeta_{i j} q_{i}-C_{j}\right)
\end{aligned}
$$

a qual denotaremos (por questão de simplicidade) apenas por $\mathcal{L}$. Portanto, devemos calcular suas derivadas parciais e as igualar a zero. Desse modo, derivando em relação aos $q_{i}$ 's e igualando a zero, temos

$$
\mathcal{L}_{q_{i}}=\log \frac{q_{i}}{p_{i}}+1+\mu+\sum_{j=1}^{M} \lambda_{j} \zeta_{i j}=0, \text { para } i=1, \ldots, n,
$$

em relação a $\mu$, temos

$$
\mathcal{L}_{\mu}=\sum_{i=1}^{n} q_{i}-1=0,
$$

e, finalmente, em relação aos $\lambda_{j}$ 's, temos

$$
\mathcal{L}_{\lambda_{j}}=\sum_{i=1}^{n} \zeta_{i j} q_{i}-C_{j}=0, \text { para } j=1, \ldots, M .
$$

Da equação (2.12) segue a seguinte igualdade:

$$
\frac{q_{i}}{p_{i}}=\exp \left\{-1-\mu-\sum_{j=1}^{M} \lambda_{j} \zeta_{i j}\right\}, i=1, \ldots, n
$$

ou seja,

$$
q_{i}=p_{i} e^{-(1+\mu)} e^{-\sum_{j=1}^{M} \lambda_{j} \zeta_{i j}}, i=1, \ldots, n
$$


de modo que restam apenas determinar $\mu$ e os $\lambda_{j}$ 's. A determinação de $\mu$ fica por conta da equação (2.13), da qual (usando (2.15)) obtemos

$$
e^{-(1+\mu)} \sum_{i=1}^{n} p_{i} e^{-\sum_{j=1}^{M} \lambda_{j} \zeta_{i j}}=1
$$

e, portanto,

$$
\mu=-\log \frac{1}{\sum_{i=1}^{n} p_{i} e^{-\sum_{j=1}^{M} \lambda_{j} \zeta_{i j}}}-1 .
$$

Consequentemente, substituindo (2.16) em (2.15),

$$
q_{i}=\frac{e^{-\sum_{j=1}^{M} \lambda_{j} \zeta_{i j}}}{\sum_{i=1}^{n} p_{i} e^{-\sum_{j=1}^{M} \lambda_{j} \zeta_{i j}}} p_{i}, i=1, \ldots, n,
$$

o que não passa de uma simples normalização. Os $\lambda_{j}$ 's são, finalmente, calculados substituindo as $n$ equações em (2.17) nas $M$ últimas equações em (2.14) e resolvendo-as para $\lambda$ (caso haja solução). ${ }^{3}$

Finalmente, podemos passar para o caso contínuo, o qual é análogo ao anterior. De fato, nosso problema agora é dado por:

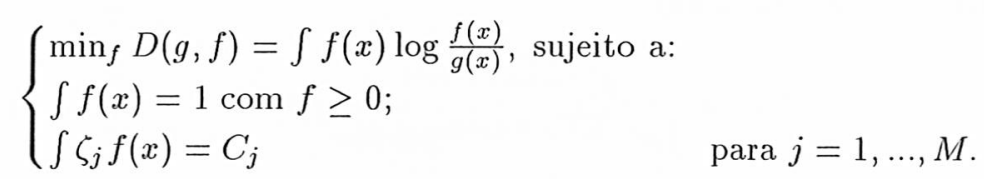

onde $\zeta_{j}$ são funções reais definidas no mesmo domínio que $f$, para todo $j=$ $1, \ldots, M$. A primeira condição apenas impõe que o espaço em que estamos trabalhando seja o espaço das funções densidade de probabilidade, enquanto que as outras $M$ condições podem ser interpretadas como (embora não necessariamente) imposições sobre os momentos da densidade minimizadora. Novamente, resolveremos esse problema via multiplicadores de Lagrange e para tal, definamos,

$J(f):=\int f(x) \log \frac{f(x)}{g(x)} d x+\sum_{j=1}^{M} \lambda_{j}\left(\int \zeta_{j}(x) f(x) d x-C_{j}\right)+\mu\left(\int f(x) d x-1\right)$

e, assumindo certas condições de regularidade temos

$$
\delta J(f ; v)=\int\left(1+\log \frac{f(x)}{g(x)}+\sum_{j=1}^{M} \lambda_{j} \zeta_{j}(x)+\mu\right) v(x) d x
$$

onde,

$$
\delta J(f ; v)=\lim _{\epsilon \rightarrow 0} \frac{J(f+\epsilon v)-J(f)}{\epsilon}
$$

${ }^{3}$ Segue do teorema da função implícita que esse sistema de equações terá solução quando o determinante da matriz jacobiana de $\mathcal{L}:=\left(\mathcal{L}_{\lambda_{1}}, \ldots, \mathcal{L}_{\lambda_{M}}, \mathcal{L}_{\mu}\right)$ em relação a $\left(\lambda_{1}, \ldots, \lambda_{M}, \mu\right)$ tiver determinante não-nulo. 
é a derivada direcional do funcional $J$ no ponto $f$ e na direção $v$, também conhecida por variação de Gâteaux ou primeira variação. Para que $f$ seja a densidade minimizadora é suficiente que $\delta J(f ; v)=0$ para todo $v$ (a existência e unicidade de tal densidade segue da convexidade da entropia relativa), logo, devemos ter:

$$
1+\log \frac{f(x)}{g(x)}+\sum_{j=1}^{M} \lambda_{j} \zeta_{j}(x)+\mu=0
$$

ou seja,

$$
f(x)=e^{-(1+\mu)} e^{-\sum_{j=1}^{M} \lambda_{j} \zeta_{j}(x)} g(x) .
$$

Agora, usando a segunda restrição em nosso programa, devemos ter:

$$
e^{-(1+\mu)} \int e^{-\sum_{j=1}^{M} \lambda_{j} \zeta_{j}(x)} g(x) d x=1
$$

ou seja

$$
e^{-(1+\mu)}=\frac{1}{\int e^{-\sum_{j=1}^{M} \lambda_{j} \zeta_{j}(x)} g(x) d x}
$$

e substituindo (2.22) em (2.21)

$$
f(x)=\frac{e^{-\sum_{j=1}^{M} \lambda_{j} \zeta_{j}(x)}}{\int e^{-\sum_{j=1}^{M} \lambda_{j} \zeta_{j}(x)} g(x) d x} g(x) .
$$

Ora, restam apenas os $\lambda_{j}$ 's $(j=1, \ldots, M)$ a serem determinados e, para tal, devemos substituir (2.23) nas $M$ últimas equações em (2.18) e resolver o sistema de equações resultante para $\lambda$.

\subsubsection{Caso Geral}

\section{Geometria Entrópica}

4

Comecemos nossa análise formalizando o problema. Seja $\mathcal{M}$ o conjunto de todas medidas de probabilidade sobre o um espaço mensurável $(X, \mathcal{B}(X))$. Então, dada uma medida de referência $\mathrm{P}_{0} \in \mathcal{M}$ e um subconjunto $\mathcal{M}^{\prime} \subset \mathcal{M}$, das medidas de probabilidade admíssiveis (ou seja, das medidas que estão de acordo com a informação disponível), nosso problema é encontrar $\mathbf{P} \in \mathcal{M}^{\prime}$ satisfazendo $D\left(\mathbf{P}_{\mathbf{0}}, \mathbf{P}\right) \leq D\left(\mathbf{P}_{\mathbf{0}}, \mathbf{Q}\right)$, para toda medida de probabilidade $\mathbf{Q} \in \mathcal{M}^{\prime}$. Em outras palavras, o que desejamos é encontrar uma distribuição de probabilidade ótima no sentido de ser a mais próxima (entropicamente) da medida de referência dentre aquelas que satisfazem certas restrições impostas pela informação disponível.

Definamos

$$
\Delta\left(\mathbf{P}_{0}, \mathcal{M}^{\prime}\right):=\inf _{\mathbf{Q} \in \mathcal{M}^{\prime}} D\left(\mathbf{P}_{0}, \mathbf{Q}\right)
$$

\footnotetext{
${ }^{4}$ As definições e resultados abaixo foram primeiramente formalizadas por Csiszár em um artigo [5] sobre a otimização e a geometria da entropia relativa.
} 
a qual representa a "distância" entrópica entre $\mathrm{P}_{\mathbf{0}}$ e $\mathcal{M}^{\prime}$. Utilizamos a notação $\Delta$ ao invés de $d$ para enfatizarmos que não estamos lidando com uma distância no sentido usual da palavra.

Suponha agora que existe $\mathbf{P} \in \mathcal{M}^{\prime}$ tal que

$$
D\left(\mathrm{P}_{0}, \mathrm{P}\right)=\Delta\left(\mathrm{P}_{\mathbf{0}}, \mathcal{M}^{\prime}\right)
$$

então, dizemos que $\mathbf{P}$ é a projeção entrópica de $\mathbf{P}_{0}$ sobre $\mathcal{M}^{\prime}$. É fácil ver que nem existência, nem unicidade da projeção entrópica são garantidas em qualquer caso. Por exemplo, se para toda medida $\mathbf{P} \in \mathcal{M}^{\prime}$ tem-se que $\mathbf{P}$ não é absolutamente contínua em relação a $\mathbf{P}_{0}$, então $D\left(\mathbf{P}_{0}, \mathbf{P}\right)=\infty$, para toda $\mathrm{P} \in \mathcal{M}^{\prime}$. O próximo resultado refere-se exatamente às circunstâncias nas quais podemos garanti-las. Note a analogia com o Teorema de Riesz para espaços de Hilbert.

Teorema 18 Lema da Projeção Entrópica. Seja $\mathcal{M}^{\prime} \subset \mathcal{M}$ um conjunto convexo tal que $\Delta\left(\mathbf{P}_{0}, \mathcal{M}^{\prime}\right)<\infty$. Então,

1. se existe a projeção entrópica de $\mathrm{P}_{\mathbf{0}}$ sobre $\mathcal{M}^{\prime}$, ela é única;

2. se $\mathcal{M}^{\prime}$ é fechado em variação, então existe a projeção entrópica $\mathbf{P}$ de $\mathbf{P}_{\mathbf{0}}$ sobre $\mathcal{M}^{\prime}$.

Prova. Provemos a primeira parte do teorema. Sejam $\mathbf{P}_{1}$ e $\mathbf{P}_{2}$ projeções entrópicas de $\mathbf{P}_{0}$ sobre $\mathcal{M}^{\prime}$. Então, pela convexidade de $\mathcal{M}^{\prime}$ tem-se que $\mathbf{P}:=$ $\frac{1}{2}\left(\mathbf{P}_{\mathbf{1}}+\mathbf{P}_{\mathbf{2}}\right) \in \mathcal{M}^{\prime}$. Então, pela convexidade da entropia relativa temos que:

$$
\Delta\left(\mathbf{P}_{\mathbf{0}}, \mathcal{M}^{\prime}\right) \leq D\left(\mathbf{P}_{0}, \mathbf{P}\right) \leq \frac{1}{2} D\left(\mathbf{P}_{0}, \mathbf{P}_{\mathbf{1}}\right)+\frac{1}{2} D\left(\mathbf{P}_{\mathbf{0}}, \mathbf{P}_{2}\right)=\Delta\left(\mathbf{P}_{\mathbf{0}}, \mathcal{M}^{\prime}\right)
$$

$\operatorname{logo} D\left(\mathbf{P}_{\mathbf{0}}, \mathbf{P}\right)=\frac{1}{2} D\left(\mathbf{P}_{\mathbf{0}}, \mathbf{P}_{\mathbf{1}}\right)+\frac{1}{2} D\left(\mathbf{P}_{\mathbf{0}}, \mathbf{P}_{\mathbf{2}}\right)$ e, consequentemente, $\mathbf{P}_{\mathbf{1}}=\mathbf{P}_{\mathbf{2}}$, pois $h$ é estritamente convexa na segunda variável.

Resta agora somente mostrarmos a parte (2) do teorema. A prova é análoga a prova de existência de projeção em espaços de Hilbert. Seja $\left(\mathbf{P}_{n}\right)_{n}$ uma sequência de medidas de probabilidade em $\mathcal{M}^{\prime}$ (note que, em particular, $\mathrm{P}_{n} \ll$ $\left.P_{0}\right)$ tal que

$$
D\left(\mathbf{P}_{0}, \mathbf{P}_{n}\right) \longrightarrow \Delta\left(\mathbf{P}_{0}, \mathcal{M}^{\prime}\right):=\delta
$$

quando $n \rightarrow \infty$, e note que:

$$
\begin{aligned}
D\left(\mathbf{P}_{\mathbf{0}}, \mathbf{P}_{m}\right)+D\left(\mathbf{P}_{\mathbf{0}}, \mathbf{P}_{n}\right)= & 2 D\left(\mathbf{P}_{\mathbf{0}}, \frac{\mathbf{P}_{m}+\mathbf{P}_{n}}{2}\right)+D\left(\frac{\mathbf{P}_{m}+\mathbf{P}_{n}}{2}, \mathbf{P}_{m}\right) \\
& +D\left(\frac{\mathbf{P}_{m}+\mathbf{P}_{n}}{2}, \mathbf{P}_{n}\right)
\end{aligned}
$$

(esta equação é a análoga à identidade do paralelogramo), onde $\frac{\mathbf{P}_{m}+\mathbf{P}_{n}}{2} \in \mathcal{M}^{\prime}$ devido à convexidade de $\mathcal{M}^{\prime}$. Note que os dois últimos termos de (2.27) devem convergir para zero quando $n \rightarrow \infty$. 
De fato, o lado esquerdo da expressão (2.27), assim como $2 h\left(\mathbf{P}_{\mathbf{0}}, \frac{\mathbf{P}_{m}+\mathbf{P}_{n}}{2}\right)$, convergem para $2 \delta$ devido à (2.26). Portanto,

$$
\lim _{m, n \rightarrow \infty}\left\{D\left(\frac{\mathbf{P}_{m}+\mathbf{P}_{n}}{2}, \mathbf{P}_{m}\right)+D\left(\frac{\mathbf{P}_{m}+\mathbf{P}_{n}}{2}, \mathbf{P}_{n}\right)\right\}=0
$$

e da não-negatividade da entropia relativa segue, portanto, que

$$
\lim _{m, n \rightarrow \infty} D\left(\frac{\mathbf{P}_{m}+\mathbf{P}_{n}}{2}, \mathbf{P}_{m}\right)=\lim _{m, n \rightarrow \infty} D\left(\frac{\mathbf{P}_{m}+\mathbf{P}_{n}}{2}, \mathbf{P}_{n}\right)=0
$$

Usando a desigualdade (1.57) tem-se que:

$$
\begin{aligned}
\left|\mathbf{P}_{m}-\mathbf{P}_{n}\right| \leq & \left|\mathbf{P}_{m}-\frac{\mathbf{P}_{m}+\mathbf{P}_{n}}{2}\right|+\left|\mathbf{P}_{n}-\frac{\mathbf{P}_{m}+\mathbf{P}_{n}}{2}\right| \\
\leq & \sqrt{2 h\left(\frac{\mathbf{P}_{m}+\mathbf{P}_{n}}{2}, \mathbf{P}_{m}\right)} \\
& +\sqrt{2 h\left(\frac{\mathbf{P}_{m}+\mathbf{P}_{n}}{2}, \mathbf{P}_{n}\right)} \longrightarrow 0
\end{aligned}
$$

quando $m, n \longrightarrow \infty$. E, portanto, $\left(\mathbf{P}_{m}\right)_{m}$ é uma sequência de Cauchy, logo existe $\mathbf{Q}$, medida de probabilidade, tal que

$$
\mathrm{P}_{n} \longrightarrow \mathrm{Q}
$$

quando $n \longrightarrow \infty$, de fato, pelo lema (5) temos que

$$
\left\|\frac{d \mathbf{P}_{m}}{d \mathbf{P}_{0}}-\frac{d \mathbf{P}_{n}}{\mathbf{P}_{0}}\right\|=\int_{X}\left|\frac{d \mathbf{P}_{m}}{d \mathbf{P}_{0}}-\frac{d \mathbf{P}_{n}}{\mathbf{P}_{0}}\right| d \mathbf{P}_{0}=\left|\mathbf{P}_{m}-\mathbf{P}_{n}\right| \longrightarrow 0
$$

quando $m, n \rightarrow \infty$. Logo, $\left(\frac{d \mathbf{P}_{n}}{d \mathbf{P}_{\mathbf{0}}}\right)_{n}$ é uma sequência de Cauchy em $L^{1}\left(\mathbf{P}_{\mathbf{0}}\right)$ e, portanto, existe $f \in L^{1}\left(\mathbf{P}_{0}\right)$ tal que

$$
\frac{d \mathbf{P}_{n}}{d \mathbf{P}_{\mathbf{0}}} \rightarrow f
$$

Defina $\mathbf{P}(A)=\int_{A} f(x) d \mathbf{P}_{0}(x), A \in \mathcal{B}(X)$.Então, $\mathbf{P} \ll \mathbf{P}_{0}$ e $\frac{d \mathbf{P}}{d \mathbf{P}_{0}}=f$. Mais ainda, $\mathbf{P}_{n} \longrightarrow_{n \rightarrow \infty} \mathbf{P}$, e como $\mathcal{M}^{\prime}$ é fechado em variação tem-se que $\mathbf{P} \in \mathcal{M}^{\prime}$. Além disso,

$$
\Delta\left(\mathbf{P}_{0}, \mathcal{M}^{\prime}\right) \leq D\left(\mathbf{P}_{0}, \mathbf{P}\right) \leq \lim _{n \rightarrow \infty} D\left(\mathbf{P}_{0}, \mathbf{P}_{n}\right)=\Delta\left(\mathbf{P}_{\mathbf{0}}, \mathcal{M}^{\prime}\right)
$$

onde na segunda desigualdade usamos o teorema (15). E, portanto, $D\left(\mathbf{P}_{\mathbf{0}}, \mathbf{P}\right)=$ $\Delta\left(\mathbf{P}_{0}, \mathcal{M}^{\prime}\right)$, ou seja, $\mathbf{P}$ é a projeção entrópica de $\mathbf{P}_{0}$ sobre $\mathcal{M}^{\prime}$.

Tendo demonstrado as condições nas quais podemos garantir a existência e a unicidade da projeção entrópica da medida de referência $P_{0}$ sobre $\mathcal{M}^{\prime}$, daremos uma caracterização dessa projeção. Para isso, definamos: 
1. $B_{r}\left(\mathbf{P}_{\mathbf{0}}\right):=\left\{\mathbf{P} \in \mathcal{M}: D\left(\mathbf{P}_{\mathbf{0}}, \mathbf{P}\right)<r\right\}$, onde $0<r \leq \infty$, o qual denominaremos bola entrópica de centro $\mathrm{P}_{0}$ e raio $r$;

2. $\mathcal{M}_{\mathbf{P}_{\mathbf{0}}, \mathbf{P}}:=\left\{\mathbf{Q} \in \mathcal{M}: \int \log \left(\frac{d \mathbf{P}}{d \mathbf{P}_{\mathbf{0}}}\right) d \mathbf{Q} \geq D\left(\mathbf{P}_{\mathbf{0}}, \mathbf{P}\right)\right\}$.

Note que, em particular, se $\mathbf{P} \in B_{r}\left(\mathbf{P}_{\mathbf{0}}\right)$, então $\mathbf{P} \ll \mathbf{P}_{\mathbf{0}}$. Além disso, $B_{r}\left(\mathbf{P}_{\mathbf{0}}\right)$ e $\mathcal{M}_{\mathbf{P}_{\mathbf{0}}, \mathbf{P}}$ são conjuntos convexos. De fato, seja $\lambda \in[0,1]$ e consideremos a medida $\mathbf{P}:=\lambda \mathbf{P}_{1}+(1-\lambda) \mathbf{P}_{\mathbf{2}}$ onde $\mathbf{P}_{\mathbf{1}}$ e $\mathbf{P}_{\mathbf{2}}$ são elementos arbitrários de $B_{r}\left(\mathbf{P}_{\mathbf{0}}\right)$. Queremos mostrar que $\mathbf{P} \in B_{r}\left(\mathbf{P}_{\mathbf{0}}\right)$. Como

$$
D\left(\mathbf{P}_{\mathbf{0}}, \mathbf{P}\right)=\int \frac{d \mathbf{P}}{d \mathbf{P}_{\mathbf{0}}} \log \left(\frac{d \mathbf{P}}{d \mathbf{P}_{0}}\right) d \mathbf{P}_{\mathbf{0}}
$$

e como a função $x \log x$ é convexa segue que:

$$
\begin{aligned}
D\left(\mathbf{P}_{\mathbf{0}}, \mathbf{P}\right) & \leq \int\left(\lambda \frac{d \mathbf{P}_{\mathbf{1}}}{d \mathbf{P}_{\mathbf{0}}} \log \left(\frac{d \mathbf{P}_{\mathbf{1}}}{d \mathbf{P}_{\mathbf{0}}}\right)+(1-\lambda) \frac{d \mathbf{P}_{\mathbf{2}}}{d \mathbf{P}_{\mathbf{0}}} \log \left(\frac{d \mathbf{P}_{\mathbf{2}}}{d \mathbf{P}_{\mathbf{0}}}\right)\right) d \mathbf{P}_{\mathbf{0}} \\
& =\lambda D\left(\mathbf{P}_{0}, \mathbf{P}_{\mathbf{1}}\right)+(1-\lambda) D\left(\mathbf{P}_{\mathbf{0}}, \mathbf{P}_{\mathbf{2}}\right) \\
& <\lambda r+(1-\lambda) r=r
\end{aligned}
$$

portanto, $\mathbf{P} \in B_{r}\left(\mathbf{P}_{\mathbf{0}}\right)$ e, consequentemente, $B_{r}\left(\mathbf{P}_{\mathbf{0}}\right)$ é um conjunto convexo. Analogamente, demonstra-se que $\mathcal{M}_{\mathbf{P}_{\mathbf{0}}, \mathbf{P}}$ é convexo. Para tal basta substituir $r$ por $\int \log \left(\frac{d \mathbf{P}}{d \mathbf{P}_{\mathbf{0}}}\right) d \mathbf{Q}$ na demonstração.

Teorema 19 Seja $\mathcal{M}^{\prime} \subset \mathcal{M}$ convexo tal que $\Delta\left(\mathbf{P}_{\mathbf{0}}, \mathcal{M}^{\prime}\right)<\infty$. Então, se $\mathbf{P} \in \mathcal{M}^{\prime} \cap B_{\infty}\left(\mathbf{P}_{0}\right)$ é uma medida que satisfaz:

$$
\mathcal{M}^{\prime} \cap B_{\infty}\left(\mathbf{P}_{0}\right) \subset \mathcal{M}_{\mathbf{P}_{0}, \mathbf{P}} .
$$

tem-se que $\mathbf{P}$ é uma projeção entrópica de $\mathbf{P}_{\mathbf{0}}$ sobre $\mathcal{M}^{\prime}$.

Prova. Seja $\mathbf{P} \in \mathcal{M}^{\prime} \cap B_{\infty}\left(\mathbf{P}_{\mathbf{0}}\right) \subset \mathcal{M}_{\mathbf{P}_{\mathbf{0}}, \mathbf{P}}$. Afirmamos que $\mathbf{P}$ é uma projeção entrópica de $\mathbf{P}_{\mathbf{0}}$ sobre $\mathcal{M}^{\prime}$, ou seja, dado qualquer $\mathbf{Q} \in \mathcal{M}^{\prime}$ tem-se $D\left(\mathbf{P}_{\mathbf{0}}, \mathbf{P}\right) \leq$ $D\left(\mathbf{P}_{0}, \mathbf{Q}\right)$.

De fato, seja $\mathbf{Q} \in \mathcal{M}^{\prime}$ qualquer. Se $D\left(\mathbf{P}_{\mathbf{0}}, \mathbf{Q}\right)=\infty$ então a afirmação acima é trivial. Portanto, vamos assumir que $D\left(\mathbf{P}_{\mathbf{0}}, \mathbf{Q}\right)<\infty$ e que, consequentemente, $\mathrm{Q} \ll \mathbf{P}_{\mathbf{0}}$. Além disso, $\mathrm{Q} \in \mathcal{M}^{\prime} \cap B_{\infty}\left(\mathbf{P}_{\mathbf{0}}\right)$ e, portanto, $\mathbf{Q} \in \mathcal{M}_{\mathbf{P}_{\mathbf{0}}, \mathrm{P}}, \operatorname{logo}$,

$$
\begin{aligned}
D\left(\mathbf{P}_{\mathbf{0}}, \mathbf{Q}\right) & =\int \log \left(\frac{d \mathbf{Q}}{d \mathbf{P}_{\mathbf{0}}}\right) d \mathbf{Q}=\int \log \left(\frac{d \mathbf{Q}}{d \mathbf{P}} \frac{d \mathbf{P}}{d \mathbf{P}_{\mathbf{0}}}\right) d \mathbf{Q} \\
& =\int \log \left(\frac{d \mathbf{Q}}{d \mathbf{P}}\right) d \mathbf{Q}+\int \log \left(\frac{d \mathbf{P}}{d \mathbf{P}_{\mathbf{0}}}\right) d \mathbf{Q} \\
& \geq D(\mathbf{P}, \mathbf{Q})+D\left(\mathbf{P}_{\mathbf{0}}, \mathbf{P}\right) \geq D\left(\mathbf{P}_{\mathbf{0}}, \mathbf{P}\right)
\end{aligned}
$$

ou seja, $D\left(\mathbf{P}_{\mathbf{0}}, \mathbf{P}\right) \leq D\left(\mathbf{P}_{0}, \mathbf{Q}\right)$ sempre que $\mathbf{Q} \in \mathcal{M}^{\prime}$, logo, $\mathbf{P}$ 'e uma projeção entrópica de $\mathbf{P}_{\mathbf{0}}$ sobre $\mathcal{M}^{\prime}$. 


\section{A Medida de Equilíbrio}

Um dos casos mais importantes, e o que nos interessa, para aplicar o método da entropia relativa mínima é aquele em que a informação disponível com relação a uma variável aleatória $S$ é dada em termos de esperanças. Ou seja, o caso em que queremos minimizar a entropia relativa de uma determinada distribuição dada a priori com relação a distribuições que, para um certo $n$ e certas funções reais mensuráveis $\zeta_{1}, \ldots, \zeta_{n}$, satisfazem restrições com relação às esperanças $\mathbf{E}\left[\zeta_{1}(S)\right], \ldots, \mathbf{E}\left[\zeta_{n}(S)\right]$ as quais são conhecidas a priori. Usaremos o último teorema para encontrar tal distribuição e, além disso, mostraremos que a mesma tem densidade do tipo exponencial com relação a medida de referência.

Para $i=1, \ldots, n$ e $\lambda=\left(\lambda_{1}, \ldots, \lambda_{n}\right)$ definamos:

$$
\theta_{i}(\lambda):=\frac{1}{\Phi(\lambda)} \mathbf{E}^{\mathbf{P}_{\mathbf{o}}}\left[\zeta_{i} \exp \left(\sum_{j=1}^{n} \lambda_{j} \zeta_{j}\right)\right]
$$

onde $\Phi(\lambda):=\mathbf{E}^{\mathbf{P}_{\mathbf{o}}}\left[\exp \left(\sum_{j=1}^{n} \lambda_{j} \zeta_{j}\right)\right]$ é um fator de normalização. Denotemos por $\Lambda$ o conjunto de todos os $\lambda$ 's para os quais as integrais acima estão bemdefinidas, e definamos o conjunto $\mathcal{A} \subset \mathbb{R}^{n}$ por:

$$
\mathcal{A}=\left\{\left(\theta_{1}(\lambda), \ldots, \theta_{n}(\lambda)\right): \lambda \in \Lambda\right\} .
$$

Temos então o seguinte resultado:

Teorema 20 Seja $\mathbf{P}_{\mathbf{o}} \in \mathcal{M}$ uma medida de referência e tomemos $\zeta_{i}(x)$, para $i=1, \ldots, n$, funções reais. Assumamos que $\mathcal{M}^{\prime} \subset \mathcal{M}$ é dado por:

$$
\mathcal{M}^{\prime}:=\left\{\mathbf{P} \in \mathcal{M}: \int \zeta_{i}(x) d \mathbf{P}(x)=C_{i}, i=1, \ldots, n\right\}
$$

onde $\left(C_{1}, \ldots, C_{n}\right) \in \mathcal{A}$ são constantes dadas. Então, a projeção entrópica de $\mathbf{P}_{0}$ sobre $\mathcal{M}^{\prime}$ existe $e$

$$
\frac{d \mathbf{P}}{d \mathbf{P}_{\mathbf{0}}}(x)=\frac{1}{\Phi(\lambda)} \exp \left(\sum_{i=1}^{n} \lambda_{i} \zeta_{i}(x)\right)
$$

onde $\lambda=\left(\lambda_{1}, \ldots, \lambda_{n}\right) \in \mathbb{R}^{n}$ é um vetor unicamente determinado pelas equações:

$$
\frac{1}{\Phi(\lambda)} \int \zeta_{i}(x) \exp \left(\sum_{j=1}^{n} \lambda_{j} \zeta_{j}(x)\right) d \mathbf{P}_{0}(x)=C_{i}
$$

para $i=1, \ldots, n$. A entropia relativa mínima correspondente é dada por:

$$
D\left(\mathbf{P}_{\mathbf{0}}, \mathbf{P}\right)=\sum_{i=1}^{n} \lambda_{i} C_{i}-\log (\Phi(\lambda))
$$


Prova. Como $\left(C_{1}, \ldots, C_{n}\right) \in \mathcal{A}$, existe um vetor $\left(\lambda_{1}, \ldots, \lambda_{n}\right) \in \Lambda$ tal que $\theta_{i}(\lambda)=$ $C_{i}$ para $i=1, \ldots, n$, ou seja, vale a fórmula (2.31). A entropia relativa da medida $\mathbf{P}$ definida em (2.30) com $\mathbf{P}_{\mathbf{0}}$ é dada por (2.32). De fato,

$$
\begin{aligned}
D\left(\mathbf{P}_{\mathbf{0}}, \mathbf{P}\right) & =\int \log \left(\frac{d \mathbf{P}}{d \mathbf{P}_{\mathbf{0}}}\right) d \mathbf{P}=\int \log \left(\frac{1}{\Phi(\lambda)} \exp \left(\sum_{i=1}^{n} \lambda_{i} \zeta_{i}(x)\right)\right) d \mathbf{P} \\
& =\sum_{i=1}^{n} \lambda_{i} \int \zeta_{i}(x) d \mathbf{P}-\int \log (\Phi(\lambda)) d \mathbf{P}=\sum_{i=1}^{n} \lambda_{i} C_{i}-\log (\Phi(\lambda))
\end{aligned}
$$

Além disso, se $\mathrm{Q}$ é uma medida em $\mathcal{M}$ então:

$$
\begin{aligned}
\int \log \left(\frac{d \mathbf{P}}{d \mathbf{P}_{\mathbf{0}}}\right) d \mathbf{Q} & =\int \log \left(\frac{1}{\Phi(\lambda)} \exp \left(\sum_{i=1}^{n} \lambda_{i} \zeta_{i}(x)\right)\right) d \mathbf{Q} \\
& =\sum_{i=1}^{n} \lambda_{i} \int \zeta_{i}(x) d \mathbf{Q}-\int \log (\Phi(\lambda)) d x
\end{aligned}
$$

e, portanto,

$$
\int \log \left(\frac{d \mathbf{P}}{d \mathbf{P}_{\mathbf{0}}}\right) d \mathbf{Q}=\sum_{i=1}^{n} \lambda_{i} C_{i}-\log (\Phi(\lambda))
$$

Por outro lado,

$$
\begin{aligned}
\mathcal{M}_{\mathbf{P}_{\mathbf{0}}, \mathbf{P}} & =\left\{\mathrm{Q} \in \mathcal{M}: \int \log \left(\frac{d \mathbf{P}}{d \mathbf{P}_{\mathbf{0}}}\right) d \mathbf{Q} \geq D\left(\mathbf{P}_{\mathbf{0}}, \mathbf{P}\right)\right\} \\
& =\left\{\mathrm{Q} \in \mathcal{M}: \sum_{i=1}^{n} \lambda_{i} \int \zeta_{i}(x) d \mathbf{Q}-\log (\Phi(\lambda)) \geq \sum_{i=1}^{n} \lambda_{i} C_{i}-\log (\Phi(\lambda))\right\} \\
& =\left\{\mathrm{Q} \in \mathcal{M}: \sum_{i=1}^{n} \lambda_{i} \int \zeta_{i}(x) d \mathbf{Q} \geq \sum_{i=1}^{n} \lambda_{i} C_{i}\right\}
\end{aligned}
$$

Porém, se $\mathbf{Q} \in \mathcal{M}^{\prime}$ então $\int \zeta_{i}(x) d \mathbf{Q}=C_{i}$ e, portanto, $\sum_{i=1}^{n} \lambda_{i} \int \zeta_{i}(x) d \mathbf{Q}=$ $\sum_{i=1}^{n} \lambda_{i} C_{i} \log \mathrm{O} \mathcal{M}^{\prime} \subset \mathcal{M}_{\mathbf{P}_{\mathbf{0}}, \mathbf{P}}$. Consequentemente, pelo último teorema $\mathbf{P}$ é a projeção entrópica de $\mathbf{P}_{\mathbf{0}}$ sobre $\mathcal{M}^{\prime}$.

No caso em que as distribuições de probabilidade são contínuas, digamos $d \mathbf{P}_{0}(x)=f_{0}(x) d x$ e $d \mathbf{P}(x)=f(x)$, então o teorema acima pode ser reescrito como:

Corolário 4 Seja $d \mathbf{P}_{\mathbf{0}}(x)=f_{0}(x) d x \in \mathcal{M}$ uma medida de referência e tomemos $\zeta_{i}(x)$, para $i=1, \ldots, n$, funções reais. Assuma que $\mathcal{M}^{\prime} \subset \mathcal{M}$ é dado por:

$$
\mathcal{M}^{\prime}:=\left\{f \in \mathcal{M}: \int \zeta_{i}(x) f(x) d x=C_{i}, i=1, \ldots, n\right\}
$$


onde $\left(C_{1}, \ldots, C_{n}\right) \in \mathcal{A}$ são constantes dadas. Então, a projeção entrópica de $f_{0}$ sobre $\mathcal{M}^{\prime}$ existe e é dada por:

$$
f(x)=\frac{1}{\Phi(\lambda)} \exp \left(\sum_{i=1}^{n} \lambda_{i} \zeta_{i}(x)\right) f_{0}(x)
$$

onde o fator de normalização agora é dado por

$$
\Phi(\lambda):=\int \exp \left(\sum_{j=1}^{n} \lambda \zeta_{j}(x)\right) f_{0}(x) d x
$$

$e \lambda=\left(\lambda_{1}, \ldots, \lambda_{n}\right) \in \mathbb{R}^{n}$ é um vetor unicamente determinado pelas equações:

$$
\frac{1}{\Phi(\lambda)} \int \zeta_{i}(x) \exp \left(\sum_{j=1}^{n} \lambda_{j} \zeta_{j}(x)\right) f_{0}(x)=C_{i}
$$

para $i=1, \ldots, n$. A entropia relativa mínima correspondente é dada por:

$$
D\left(f_{0}, f\right)=\sum_{i=1}^{n} \lambda_{i} C_{i}-\log (\Phi(\lambda))
$$

E, analogamente, para distribuições discretas:

Corolário 5 Seja $\mathbf{p}=\left(p_{1}, \ldots, p_{m}\right) \in \mathcal{M}$ uma medida de referência e tomemos $\zeta_{i}(x)$, para $i=1, \ldots, n$, funções reais. Assuma que $\mathcal{M}^{\prime} \subset \mathcal{M}$ é dado por:

$$
\mathcal{M}^{\prime}:=\left\{\mathrm{q} \in \mathcal{M}: \sum_{j=1}^{m} \zeta_{i j} p_{j}=C_{i}, i=1, \ldots, n\right\}
$$

onde $\left(C_{1}, \ldots, C_{n}\right) \in \mathcal{A}$ são constantes dadas. Então, a projeção entrópica de $\mathbf{p}$ sobre $\mathcal{M}^{\prime}$ existe e é dada por:

$$
q_{j}=\frac{1}{\Phi(\lambda)} \exp \left(\sum_{i=1}^{n} \lambda_{i} \zeta_{i j}\right) p_{j}
$$

onde o fator de normalização agora é dado por

$$
\Phi(\lambda):=\sum_{j=1}^{m} \exp \left(\sum_{i=1}^{n} \lambda \zeta_{i j}\right) p_{j}
$$

e $\lambda=\left(\lambda_{1}, \ldots, \lambda_{n}\right) \in \mathbb{R}^{n}$ é um vetor unicamente determinado pelas equações:

$$
\frac{1}{\Phi(\lambda)} \sum_{j=1}^{m} \zeta_{i j} \exp \left(\sum_{k=1}^{n} \lambda_{k} \zeta_{k j}\right) p_{j}=C_{i}
$$

para $i=1, \ldots, n$. A entropia relativa mínima correspondente é dada por:

$$
D(\mathbf{p}, \mathbf{q})=\sum_{i=1}^{n} \lambda_{i} C_{i}-\log (\Phi(\lambda))
$$




\subsection{Maximizando a Entropia}

Analisemos um outro problema, mas que mantém uma conexão com o estudado até aqui: a maximização da entropia. Evidentemente, se não impomos nenhuma restrição a essa distribuição, então, pelo teorema (1), a distribuição que maximiza a entropia é a uniforme. No entanto, e se restrições são impostas? Resolveremos esse problema tanto no caso contínuo como no caso discreto. Dessa maneira, começaremos, assim como na penúltima seção, com o caso discreto determinando diretamente a distribuição de probabilidades que maximiza a entropia. No entanto, não resolveremos o problema explicitamente via multiplicadores de Lagrange como fizemos no caso da entropia relativa, pois, conhecendo a distribuição que minimiza a entropia relativa dada uma distribuição a priori (no caso, a distribuição uniforme), é mais fácil e imediato usarmos a relação (1.45) que relaciona os conceitos de entropia e entropia relativa. Analisemos, portanto, o seguinte problema de otimização:

Para $n$ fixado:

$$
\begin{cases}\max _{\mathbf{q}}-\sum_{i=1}^{n} q_{i} \log q_{i} & \text { onde } \mathbf{q}=\left(q_{1}, \ldots, q_{n}\right) ; \\ \sum_{i}^{n} q_{i}=1 ; & \text { para } j=1, \ldots, M ; \\ \sum_{i}^{n} \zeta_{i j} q_{i}=C_{j} & \end{cases}
$$

onde $\zeta_{i j} \in \mathbb{R}$, para todo $j=1, \ldots, M$. Ora, a relação (1.45) afirma que:

$$
h(\mathbf{q})=-D(\mathbf{u}, \mathbf{q})+\log n,
$$

onde $\mathbf{u}$ é a distribuição uniforme, logo, resolver o programa em (2.39) equivale a minimizar a entropia relativa com a distribuição dada a priori igual a distribuição uniforme $\mathbf{u}=\left(\frac{1}{n}, \ldots, \frac{1}{n}\right)$, cuja solução já conhecemos e é dada pela relação $(2.17) \operatorname{com} p_{i}=\frac{1}{n}$ para $i=1, \ldots, n$. Portanto, devemos ter

$$
q_{i}=\frac{e^{\sum_{j=1}^{M} \lambda_{j} \zeta_{i j}}}{\frac{1}{n} \sum_{i=1}^{n} e^{\sum_{j=1}^{M} \lambda_{j} \zeta_{i j}}} \frac{1}{n}, i=1, \ldots, n,
$$

ou seja,

$$
q_{i}=\frac{e^{\sum_{j=1}^{M} \lambda_{j} \zeta_{i j}}}{\sum_{i=1}^{n} e^{\sum_{j=1}^{M} \lambda_{j} \zeta_{i j}}}, i=1, \ldots, n
$$

restando apenas determinar os $\lambda_{j}$ 's ótimos, os quais são, finalmente, calculados substituindo as $n$ equações em (2.41) nas $M$ últimas equações em (2.39) e resolvendo-as para $\lambda$.

No caso contínuo, a situação não é tão imediata, pois, como veremos agora, se procedemos da forma acima a função densidade de probabilidade $f$ resultante que maximiza a entropia depende do suporte da função densidade de probabilidade uniforme dada, ou seja, essa informação é incorporada na densidade $f$. Logo, apesar da relação (1.46) (contra-parte da relação (1.45) no caso contínuo) ser verdadeira e apesar de conhecermos a densidade de probabilidade que minimiza a entropia relativa dada qualquer densidade uniforme a priori, deveremos, 
se quisermos encontrar a densidade que maximiza a entropia num caso geral onde as únicas restrições são aquelas dadas no programa, resolver o problema explicitamente desde o princípio. Para efeito de comparação e ilustração, vamos, em primeiro lugar, encontrar a densidade ótima de modo similar ao feito no caso discreto, ou seja, dada a seguinte densidade uniforme

$$
u(x)= \begin{cases}\frac{1}{b-a} & \text { se } x \in(a, b) ; \\ 0 & \text { caso contrário }\end{cases}
$$

onde $a, b \in \mathbb{R}$ e $b>a$, resolver o problema

$$
\left\{\begin{array}{lc}
\max _{f} h(f)=\int f(x) \log f(x) d x, & \text { sujeito a: } \\
\int f(x)=1 & \text { com } f \geq 0 \\
\int \zeta_{j} f(x)=C_{j} & \text { para } j=1, \ldots, M
\end{array}\right.
$$

equivale a encontrar a função densidade de probabilidade $f$ que minimiza a entropia relativa $D(u, f)$ e satisfaz as $M$ condições $\int \zeta_{j} f(x)=C_{j}(j=1, \ldots, M)$ devido à relação (1.46) a qual afirma que

$$
h(f)=-D(u, f)+\log (b-a) .
$$

Ora, essa solução é conhecida e é dada pela relação (2.23), logo, devemos ter

$$
f(x)=\frac{e^{-\sum_{j=1}^{M} \lambda_{j} \zeta_{j}(x)}}{\int e^{-\sum_{j=1}^{M} \lambda_{j} \zeta_{j}(x)} u(x) d x} u(x) .
$$

ou seja,

$$
f(x)= \begin{cases}\frac{e^{-\sum_{j=1}^{M} \lambda_{j} \zeta_{j}(x)}}{\int_{a}^{b} e^{-\sum_{j=1}^{M} \lambda_{j} \zeta_{j}(x)} d x}, & \text { se } x \in(a, b) ; \\ 0, & \text { caso contrário; }\end{cases}
$$

ficando, dessa forma, evidente que a informação trazida pela densidade uniforme de que qualquer evento fora do intervalo $(a, b)$ tem probabilidade nula de ocorrer.

Para analisar o caso mais geral onde tal informação não está disponível, vamos deduzir a densidade de probabilidade que maximiza a entropia desde o prinípio, de forma análoga a feita para a entropia relativa no caso contínuo. Queremos resolver o programa (2.42), então, definamos o seguinte funcional:

$$
\begin{aligned}
J(f):= & -\int f(x) \log f(x) d x \\
& +\mu\left(\int f(x) d x-1\right)+\sum_{i=1}^{M} \lambda_{i}\left(\int \zeta(x) f(x) d x-C_{i}\right)
\end{aligned}
$$

e assumindo as mesmas condições de regularidade que no caso da entropia rela- 
tiva, temos

$$
\begin{aligned}
\delta J(f ; v) & =\lim _{\epsilon \rightarrow 0} \frac{J(f+\epsilon v)-J(f)}{\epsilon} \\
& =-\int\left(\log f(x)+1-\mu-\sum_{i=1}^{M} \lambda_{i} \zeta(x)\right) v(x) d x \\
& =0
\end{aligned}
$$

para toda $v$ se, e somente se,

$$
\log f(x)+1-\mu-\sum_{i=1}^{M} \lambda_{i} \zeta(x)=0
$$

ou seja se, e somente se,

$$
f(x)=e^{1-\mu} e^{-\sum_{i=1}^{M} \lambda_{i} \zeta(x)}
$$

Agora, substiuindo (2.45) na restrição $\int f(x) d x=1$ obtemos

$$
e^{1-\mu}=\frac{1}{\int e^{-\sum_{i=1}^{M} \lambda_{i} \zeta(x)}}
$$

e, consequentemente,

$$
f(x)=\frac{e^{-\sum_{i=1}^{M} \lambda_{i} \zeta(x)}}{\int e^{-\sum_{i=1}^{M} \lambda_{i} \zeta(x)}}
$$

onde os lambda $a_{i}$ 's, para $i=1 \ldots, M$, são calculados, como sempre, substituindoos nas $M$ últimas restrições do nosso programa.

\subsection{Aplicação do Princípio Entrópico}

Voltemos à consideração do problema de inferência da distribuição de ArrowDebreu. Vamos supor que existem dois ativos, um bond livre-de-risco e uma ação representando o ativo de risco. Como já dissemos mais acima deve-se em primeiro lugar checar qual é a informação a disposição, ou seja, deve-se decidir quais são o espaço de probabilidades com o qual modelamos o mercado (a princípio assumiremos que as distribuições são discretas e que, consequentemente, moram em algum simplex contido em um espaço euclidiano de dimensão finita $U$, de grande interesse para a modelagem do mercado via árvores multinomiais), o preço de um bond padrão livre-de-risco, $\pi_{0}$ que no final do período paga uma unidade monetária, o preço corrente da ação, $S$ e, finalmente, a volatilidade do preço da ação (estimada a priori, através dos dados disponíveis no mercado). Obviamente, se o número de estados $M$ for maior do que 2, estamos numa situação de mercado incompleto e o investidor tem uma variedade de distribuições para escolher. Pela fórmula (2.6), se queremos que a distribuição resultante seja de Arrow-Debreu, devemos ter a seguinte condição satisfeita: 
$\sum_{i=1}^{U} p_{i} S_{i}=\frac{S}{P}$. Ora, então, pelo princípio entrópico, podemos escrever esse problema da seguinte forma:

$$
\max _{\mathbf{p}}-\sum_{i=1}^{U} p_{i} \log p_{i}
$$

sujeito a:

$$
\begin{cases}\sum_{i=1}^{U} p_{i}=1 & \text { com } p_{i}>0 \\ \sum_{i=1}^{U} p_{i} S_{i}=\frac{S}{P} & \\ \sum_{i=1}^{U} p_{i} S_{i}^{2}=\sigma^{2}+\frac{S^{2}}{P^{2}} & \end{cases}
$$

onde a última restrição representa uma aposta do investidor ou um consenso do mercado com relação à volatilidade do preço da ação.

Ora, mas a solução desse problema é conhecida e se a distribuição for denotada por $\mathrm{p}^{*}$ ela é dada por:

$$
p_{i}^{*}=\frac{e^{\lambda_{1}^{*} S_{i}+\lambda_{2}^{*} S_{i}^{2}}}{\sum_{i=1}^{U} e^{\lambda_{1}^{*} S_{i}+\lambda_{2}^{*} S_{i}^{2}}}
$$

onde $i=1, \ldots, U$ e $\lambda_{1}^{*}$ e $\lambda_{2}^{*}$ são determinados substituindo a expressão de $p_{i}^{*}$ nas duas últimas restrições do programa acima. Se denotarmos a taxa de juros livre-de-risco por $r$, então, o preço do bond é igual a $e^{-r T}$ e, portanto, a segunda restrição do programa pode ser reescrita como $\sum_{i=1}^{U} p_{i} S_{i}=e^{r T} S$ e a terceira como $\sum_{i=1}^{U} p_{i} S_{i}^{2}=\sigma^{2}+e^{2 r T} S^{2}$.

Observemos que a hipótese de não-arbitragem, que nós assumimos no início da seção, não era, na realidade, necessária, pois, ela pode ser estabelecida como uma consequência do princípio entrópico como mostra o próximo resultado. De fato, seguindo a mesma notação utilizada até agora onde temos $U$ estados, $N$ ativos (sendo um deles um ativo livre-de-risco (um bond) cujos payoffs nos diversos estados são iguais a 1 e cujo preço é $P$ ), $\pi$ como o vetor de preços dos ativos e $\mathrm{X}$ como a matriz de payoffs e definindo

$$
\zeta(\mathrm{X}):=\left(\begin{array}{c}
\zeta_{1}(\mathrm{X}) \\
\vdots \\
\zeta_{M}(\mathrm{X})
\end{array}\right)
$$

onde $\zeta_{i}(\mathbf{X})=\left(\zeta_{i 1}(\mathbf{X}), \ldots, \zeta_{i U}(\mathbf{X})\right)$ é uma função matricial assumindo valores em $\mathbb{R}^{U}$ para todo $i=1, \ldots, M$, temos

Teorema 21 Se vale o princípio da máxima entropia, então não há oportunidades de arbitragem.

Prova. Se vale o princípio da máxima entropia, então, a distribuição de probabilidades neutra ao risco desse mercado é a solução de um programa do seguinte tipo:

$$
\max _{\mathbf{p}}-\sum_{i=1}^{U} p_{i} \log p_{i}
$$


sujeito a:

$$
\left\{\begin{array}{l}
\mathrm{p}^{T} \mathbf{1}=1 \\
\mathrm{Xp}=\frac{\pi}{P} \\
\zeta(\mathrm{X}) \mathrm{p}=C
\end{array}\right.
$$

onde

$$
1:=\left(\begin{array}{c}
1 \\
\vdots \\
1
\end{array}\right)
$$

é um vetor $U$-dimensional e

$$
C:=\left(\begin{array}{c}
C_{1} \\
\vdots \\
C_{M}
\end{array}\right) \in \mathbb{R}^{M} .
$$

Ora, sabemos que se tal problema de otimização tem solução (cuja unicidade é garantida pela estrita concavidade da entropia) ela é dada por

$$
p_{j}^{*}=\frac{e^{\mu^{* T} \mathbf{X} \cdot j+\lambda^{* T} \zeta \cdot j}(\mathbf{X})}{\sum_{i=1}^{U} e^{\mu^{* T} \mathbf{X} \cdot j+\lambda^{* T} \zeta \cdot j(\mathbf{X})}}
$$

para $j=1, \ldots, U$ e onde $\mathbf{X}_{\cdot j}=\left(X_{1 j}, \ldots, X_{N j}\right)^{T}, \zeta_{\cdot j}=\left(\zeta_{1 j}(\mathrm{X}), \ldots, \zeta_{M j}(\mathrm{X})\right)^{T}$ e os multiplicadores de Lagrange

$$
\mu^{*}=\left(\begin{array}{c}
\mu_{1}^{*} \\
\vdots \\
\mu_{N}^{*}
\end{array}\right)
$$

$\mathrm{e}$

$$
\lambda^{*}=\left(\begin{array}{c}
\lambda_{1}^{*} \\
\vdots \\
\lambda_{M}^{*}
\end{array}\right)
$$

são determinados nas $N+M$ últimas restrições do programa acima substituindo a distribuição $\mathbf{p}$ pelo vetor cujas componentes são as dadas pela equação (2.48) e resolvendo-as para $\mu$ e $\lambda$.

Ora, como já observamos na equação (2.8), se temos um ativo livre-derisco e uma distribuição de probabilidades que satisfaz $\mathbf{X} \mathbf{p}=\frac{\pi}{P}$, então temos, necessariamente, um vetor de preços de estados e, consequentemente, vale o princípio da não-arbitragem pelo teorema (17). 


\section{Distribuições de Arrow-Debreu em Economias de Um Período com Espaço de Estados Contínuo}

Sejam $S_{T}^{(i)}, i=1, \ldots, N$, os preços de $N$ ativos quaisquer no tempo $T$, e tomemos as funções $G_{i}=G_{i}\left(S_{T}^{(i)}\right)$ pelos payoffs desses ativos (por exemplo, no caso de opções de compra européias com vencimentos na data $T$ e preços de exercício $K_{i}$ temos $\left.G_{i}\left(S_{i}\right)=\left(S_{T}^{(i)}-K_{i}\right)^{+}\right)$. Suponhamos também que existe dentre esses ativos um que é sem risco (ou seja, cujo payoff independe do estado do mundo) e cujo preço é $P$, então, no mesmo espírito do descrito na seção relativa aos estados discretos, consideraremos que uma função densidade de probabilidade $f$ é neutra ao risco se os valores descontados das médias dos payoffs, $G_{i}=G_{i}\left(S_{T}^{(i)}\right)$, dos ativos considerados são iguais aos preços correntes dos ativos $C_{i}$, isto é, se

$$
\int G_{i}(s) f(s) d s=\frac{C_{i}}{P} .
$$

No caso de mercados incompletos, existirão mais de uma função densidade de probabilidade neutra ao risco, cabendo aos investidores escolherem dentre todas possibilidades. No entanto, se assumimos o princípio da máxima entropia a unicidade de solução do problema de inferência da função densidade de probabilidade neutra ao risco torna-se uma consequência. De fato, tal densidade é extraida de um programa de otimização do tipo (2.42), cujas existência, unicidade e expressão da solução (ver fórmula (2.46)) são conhecidas.

De fato, consideremos o exemplo (já construido mais acima no caso discreto) onde o ativo é uma ação $S_{1}$ e um ativo neutro ao risco, cujo preço é $P$. O problema consiste em inferir a medida de probabilidade neutra ao risco "correta". Vamos novamente supor que o mercado entrou em um consenso com relação à volatilidade da ação, $\sigma^{2}$. Pelo princípio da máxima entropia, o(s) investidor(es) deve $(\mathrm{m})$ resolver o seguinte programa de otimização:

$$
\max _{f}-\int f(s) \log f(s) d s
$$

sujeito a:

$$
\left\{\begin{array}{l}
f \geq 0 \\
\int f(s) d s=1 \\
\int s f(s) d s=\frac{S_{1}}{P} \\
\int s^{2} f(s) d s=\sigma^{2}+\frac{S_{1}^{2}}{P^{2}}
\end{array}\right.
$$

cuja solução é dada por (ver (2.46)):

$$
f^{*}(s)=\frac{e^{-\lambda_{1}^{*} s-\lambda_{2}^{*} s^{2}}}{\int e^{-\lambda_{1}^{*} s-\lambda_{2}^{*} s^{2}} d s}
$$

onde $\lambda_{1}^{*}$ e $\lambda_{2}^{*}$ são calculados substituindo a expressão de $f^{*}$ acima nas duas últimas restrições do programa de otimização e resolvendo-as para $\lambda_{1}^{*}$ e $\lambda_{2}^{*}$. 
No exemplo acima não consideramos nenhuma hipótese com relação aos limites superior e inferior do preço da ação. Suponhamos que antes de qualquer outra informação ou restrição ser incorporada, sabe-se apenas que o preço da mesma não pode ir além de certos limites inferior, $S_{\min }$ (por exemplo, o preço não pode ser negativo), e superior, $S_{\max }$, mas que não se tem nenhuma noção de como se comportará dentro dessa faixa. Para incorporarmos essa informação em nosso modelo e, ainda assim, "mantermos"o estado de ignorância em que encontram-se os investidores com relação ao comportamento dos preços da ação dentro dessa faixa nós usamos uma função densidade de probabilidade a priori dada por

$$
u(s)= \begin{cases}\frac{1}{S_{\max }-S_{\min }} & \text { se } s \in\left(S_{\min }, S_{\max }\right) \\ 0 & \text { caso contrário }\end{cases}
$$

ou seja, com isso estamos apenas dizendo que a probabilidade de qualquer evento fora daquela faixa não tem nenhuma chance de ocorrência e que para os investidores, qualquer preço dentro da mesma faixa tem a mesma probabilidade de ocorrer, refletindo sua ignorância. No entanto, suponhamos agora que a mesma informação do exemplo acima foi obtida, isto é, que o mercado entrou em um consenso relativo à volatilidade e que os investidores desejam inferir uma densidade neutra ao risco que leve em consideração essa nova informação e que, obviamente, incorpore também aquelas que já eram conhecidas e estão embutidas na densidade uniforme $u$. Então, podemos usar a contra-parte do princípio da máxima entropia, ou seja, o princípio da mínima entropia relativa. Desse modo, o problema dos investidores consiste agora em resolver o seguinte programa de otimização:

sujeito a:

$$
\min _{f}-\int_{S_{\min }}^{S_{\max }} f(s) \log \frac{f(s)}{u(s)} d s
$$

$$
\left\{\begin{array}{l}
f \geq 0 \\
\int f(s) d s=1 \\
\int s f(s) d s=\frac{S_{1}}{P} \\
\int s^{2} f(s) d s=\sigma^{2}+\frac{S_{1}^{2}}{P^{2}}
\end{array}\right.
$$

cuja solução é dada por (ver (2.43)):

$$
f^{*}(s)= \begin{cases}\frac{e^{-\lambda_{1}^{*} s-\lambda_{2}^{*} s^{2}}}{\int_{\mathcal{S}_{\min }}^{\mathcal{S}_{\max }} e^{-\lambda \hat{i}^{*} s-\lambda_{2}^{*} s^{2}} d x}, & \text { se } s \in\left(S_{\min }, S_{\max }\right) ; \\ 0, & \text { caso contrário; }\end{cases}
$$

onde $\lambda_{1}^{*}$ e $\lambda_{2}^{*}$ são calculados substituindo a expressão de $f^{*}$ acima nas duas últimas restrições do programa de otimização e resolvendo-as para os mesmos. Notemos que ao introduzir o prior, $u$, acabamos por concentrar a nova medida neutra ao risco obtida entropicamente no intervalo $\left(S_{\min }, S_{\max }\right)$.

Consideremos agora um outro caso, imaginemos uma cesta de $M$ opções cujo ativo subjacente seja, por exemplo, uma ação com a mesma descrição do exemplo 
anterior e um ativo neutro ao risco, cujo preço é $P$. Todas opções vencem na data $T$, seus preços $C_{1}, \ldots, C_{M}$ e preços de exercício $K_{1}, \ldots, K_{M}$ podem ser lidos no mercado e seus payoffs são representados por $G_{1}(s), \ldots, G_{M}(s)$ (por exemplo, se a $i$-ésima opção for uma opção de compra européia, então, $\left.G_{i}(s)=\left(s-K_{i}\right)^{+}\right)$). Os objetivos dos investidores, agora, são inferir a densidade neutra ao risco e, então, a partir dessa informação, inferir a volatilidade do ativo base. Como sempre, eles devem, devido ao princípio entrópico resolver o seguinte programa de otimização:

$$
\max _{f}-\int f(s) \log f(s) d s
$$

sujeito a:

$$
\left\{\begin{array}{l}
f \geq 0 \\
\int f(s) d s=1 \\
\int G_{i}(s) f(s) d s=\frac{C_{i}}{P} \quad \text { para } i=1, \ldots, M
\end{array}\right.
$$

cuja solução é:

$$
f^{*}(s)=\frac{e^{-\sum_{i=1}^{M} \lambda_{i}^{*} G_{i}(s)}}{\int e^{-\sum_{i=1}^{M} \lambda_{i}^{*} G_{i}(s)} d s}
$$

sendo que os $\lambda_{i}^{*}$ 's são determinados utilizando a segunda restrição do programa de otimização. Tendo inferido a densidade ótima $f^{*}$, a volatilidade do ativo base é obtida pela fórmula:

$$
\sigma^{2}=\int s^{2} f^{*}(s) d s-\left(\int s f^{*}(s) d s\right)^{2}
$$

\subsection{Núcleo de Apreçamento e Entropia}

Muito da teoria financeira consiste na determinação do núcleo de apreçamento ${ }^{5}$ para um determinado ativo. Como veremos mais abaixo, o conhecimento do núcleo de apreçamento permite aos agentes o apreçamento de ativos. Mais ainda, conhecendo o núcleo de apreçamento, é possível fazer correções de risco, ou seja, determinar o retorno esperado de um certo investimento para um dado nível de risco. ${ }^{6}$ Existe uma série de outras aplicações para o núcleo de apreçamento, portanto, métodos para sua determinação são importantes para a teoria financeira. O que nós fazemos nessa seção é sugerir um núcleo de apreçamento que seja compatível com o enfoque informacional do mercado.

Suponhamos um mercado em tempo discreto com um horizonte de tempo $T$ que seja modelado por um espaço de probabilidade $(\Omega, \mathcal{B}, \mathbf{P})$ e por uma filtração $\mathcal{I}_{t}$ a qual representa a informação disponível no tempo $t$. Suponhamos também a existência de $N$ ativos, os quais denotaremos por $i=1, \ldots, N$, e cujos payoffs

\footnotetext{
${ }^{5} \mathrm{Na}$ literatura, o núcleo de apreçamento também é conhecido por Fator de Desconto Estocástico. Em seu livro [3], Cochrane introduz e desenvolve logo no primeiro capítuloo conceito de Fator de Desconto Estocástico.

${ }^{6}$ Os retornos esperados são proporcionais a covariância dos retornos com o núcleo de apreçamento.
} 
sejam representados por processos estocásticos $X_{t}^{i}$. Suponhamos também que tais variáveis aleatórias sejam adaptadas a filtração $\mathcal{I}_{t}$. O preço de cada ativo $i$ no instante $t$ é dado pelo processo estocástico $p_{t}^{i}$. O Núcleo de Apreçamento ou Fator de Desconto Estocástico é o processo $m_{t}$ tal que para qualquer ativo $i=1, \ldots, N$ tem-se:

$$
p_{t}^{i}=\mathbf{E}^{\mathrm{P}}\left[m_{t} X_{t+1}^{i} \mid \mathcal{I}_{t}\right]
$$

ou mais simplesmente,

$$
p_{t}^{i}=\mathbf{E}_{t}^{\mathrm{P}}\left[m_{t} X_{t+1}^{i}\right]
$$

O retorno bruto real do i-ésimo ativo é definido por $R_{t+1}^{i}=\frac{X_{t+1}^{i}}{p_{t}^{i}}$. Então, dividindo a equação (2.50) por $p_{t}^{i}$ obtemos:

$$
1=\mathbf{E}_{t}^{\mathbf{P}}\left[m_{t} R_{t+1}^{i}\right]
$$

e, então, tomando a esperança em ambos os lados da equação acima temos:

$$
1=\mathbf{E}^{\mathrm{P}}\left[m_{t} R_{t+1}^{i}\right]
$$

para $i=1, \ldots, N$

Consideremos agora a existência de um ativo livre de risco, o qual denotaremos por $i=0$. Esse ativo gera um retorno livre de risco o qual denotaremos por $R_{0}$. Como não há risco nesse retorno então ele é um retorno certo e, consequentemente, se assumimos, sem perda de generalidade, que seu preço é 1 , então por (2.51):

$$
\mathbf{E}^{\mathrm{P}}\left[m_{t}\right]=\frac{1}{R_{0}}
$$

Consequentemente, multiplicando a equação (2.51) por $R_{0}$, o núcleo de $m$ deve satisfazer as seguintes $N+1$ restrições:

$$
\mathbf{E}^{\mathbf{P}}\left[\frac{m_{t}}{\mathbf{E}^{\mathbf{P}}\left[m_{t}\right]} R_{t+1}^{i}\right]=R_{0}
$$

para $i=1, \ldots, N$, mais aquela dada pela equação (2.52). As equações em (2.53) podem ser reescritas como:

$$
\mathbf{E}^{\mathbf{Q}}\left[R_{t+1}^{i}\right]=R_{0}
$$

efetuando a mudança de medida:

$$
d \mathbf{Q}=\frac{m_{t}}{\mathbf{E}^{\mathrm{P}}\left[m_{t}\right]} d \mathbf{P}
$$

Muito da teoria de apreçamento de ativos consiste em determinar um núcleo de apreçamento que ajuste-se aos dados. Para isso costuma-se impor condições sobre o núcleo de apreçamento, sendo a mais comum a exigência de que o núcleo de apreçamento seja aquele de mínima variância dentre todas as possibilidades. O que nós propomos agora é o uso da teoria da informação, através do princípio da máxima entropia, para a realização de tal tarefa. Ao determinar $m$ dessa 
maneira encaramos $\mathbf{P}$ como uma medida de probabilidade obtida a priori ou, mais concisamente, como nosso prior. Desse modo, procuraremos determinar a medida $\mathbf{Q}$ que encontra-se informacionalmente mais próxima de $\mathbf{P}$ e, a partir dela, determinar $m$. Portanto, devemos resolver o seguinte programa:

$$
\min _{\frac{d \mathbf{Q}}{d \mathbf{P}}} D(\mathbf{P}, \mathbf{Q})=\mathbf{E}^{\mathbf{Q}}\left[\log \frac{d \mathbf{Q}}{d \mathbf{P}}\right]
$$

sujeito a:

$$
\mathrm{E}^{\mathrm{Q}}\left[R_{t+1}^{i}\right]=R_{0} \text { para } i=1, \ldots, N .
$$

cuja solução é dada por:

$$
\frac{d \mathbf{Q}}{d \mathbf{P}}=\frac{\exp \left(\sum_{i=1}^{N} \lambda_{i}^{*} R_{t+1}^{i}\right)}{\mathbf{E}^{\mathbf{P}}\left[\exp \left(\sum_{i=1}^{N} \lambda_{i}^{*} R_{t+1}^{i}\right)\right]}
$$

onde os multiplicadores de Lagrange $\lambda_{i}$ 's ótimos são determinados resolvendo-se para $\lambda$ o sistema não-linear formado pelas $N$ seguintes equações

$$
\mathbf{E}^{\mathbf{P}}\left[R_{t+1}^{i} \frac{d \mathbf{Q}}{d \mathbf{P}}\right]=R_{0} \text { para } i=1, \ldots, N .
$$

Então, pelas relações (2.52) e (2.55) temos que

$$
m_{t}=\frac{\exp \left(\sum_{i=1}^{N} \lambda_{i}^{*} R_{t+1}^{i}\right)}{\mathbf{E}^{\mathbf{P}}\left[\exp \left(\sum_{i=1}^{N} \lambda_{i}^{*} R_{t+1}^{i}\right)\right] R_{0}} .
$$

\subsection{Entropia e o Modelo de Black-Scholes}

Pretendemos mostrar nessa seção que a medida de Arrow-Debreu induzida pelo princípio da mínima entropia relativa coincide com a medida neutra ao risco deduzida na teoria de Black-Scholes. Observemos, no entanto, que nenhuma hipótese é feita em relação à completude do mercado. Modelaremos a incerteza através do espaço de probabilidade $(\Omega, \mathcal{B}, \mathbf{P})$, onde $\mathcal{B}$ é uma $\sigma$-álgebra sobre $\Omega$ e $\mathbf{P}$ uma medida de probabilidade e, além disso, modelaremos o processo de preços de um ativo pelo modo usual, ou seja, através do processo

$$
\frac{d S(t)}{S(t)}=\mu d t+\sigma d W(t)
$$

onde $W=W(t)$ é o processo de Wiener unidimensional. Para nossos propósitos é mais conveniente trabalharmos com sua aproximação em tempo discreto

$$
\begin{aligned}
\frac{\Delta S_{n}}{S_{n}} & =\mu \Delta t+\sigma \Delta W_{n} \\
& =\mu \Delta t+\sigma \epsilon_{n} \sqrt{\Delta t}
\end{aligned}
$$


onde $\Delta S_{n}=S_{n+1}-S_{n}$ e $\epsilon_{n}$ é uma variável aleatória (choque) com distribuição $\mathcal{N}(0,1)$ no instante $n$, logo, $\Delta W_{n}$ tem média igual a 0 e variância igual a $\Delta t$. Portanto, $\epsilon_{n} \sqrt{\Delta t} \sim \mathcal{N}(0, \Delta t)$ são choques aleatórios que modelam a evolução do retorno no tempo (discreto).

Ora, se o horizonte de tempo é dado por $T$, então, supondo uma discretização em $N$ períodos, temos $\Delta t=\frac{T}{N}$. Sejam $\pi_{n}$ a probabilidade condicional dado $S_{n-1}$, ou seja, o preço do ativo no $(n-1)$-ésimo período e $r$ a taxa livre-derisco, então, por neutralidade ao risco devemos ter

$$
\mathrm{E}^{\pi_{n}}\left[\frac{\Delta S}{S}\right]=r \Delta t
$$

e tomando a derivada de Radon-Nikodym, temos

$$
\mathbf{E}\left[\frac{\Delta S}{S} \frac{d \pi_{n}}{d \mathbf{P}}\right]=r \Delta t
$$

Agora, consideremos as $N$ densidades $\frac{d \pi_{n}}{d \mathbf{P}}$ e suas respectivas medidas $\mathbf{P}_{n}$ associadas. A transição de um instante para o seguinte representa uma economia de um período e, estando no $n$-ésimo instante, o problema consiste agora em determinar a expressão de $\frac{d \pi_{n}}{d \mathbf{P}}$ para cada $n=1, \ldots, N$. Faremos isso aplicando o princípio da mínima entropia relativa, ou seja, transformamos nosso problema em um problema de otimização não-linear dado por

$$
\min _{\frac{d \pi_{n}}{d \mathbf{P}}} \int \log \frac{d \pi_{n}}{d \mathbf{P}} d \pi_{n}
$$

sujeito a:

$$
\mathbf{E}\left[\frac{\Delta S}{S} \frac{d \pi_{n}}{d \mathbf{P}}\right]=r \Delta t
$$

Como já vimos na seção (2.3.2) a solução desse programa é

$$
\frac{d \pi_{n}}{d \mathbf{P}}(x)=\frac{e^{\lambda_{n}^{*} \frac{\Delta S}{S}}}{\mathbf{E}^{\mathbf{P}}\left[e^{\lambda_{n}^{*} \frac{\Delta S}{S}}\right]}
$$

onde

$$
\lambda_{n}^{*}=\operatorname{argmax}_{\lambda_{n}}\left\{\lambda_{n} r \Delta t-\log \mathrm{E}^{\mathrm{P}}\left[e^{\lambda_{n} \frac{\Delta S}{S}}\right]\right\} .
$$

Agora substituindo a equação (2.59) na equação (2.63),

$$
\begin{aligned}
\lambda_{n}^{*} & =\operatorname{argmax}_{\lambda_{n}}\left\{\lambda_{n} r \Delta t-\log \mathbf{E}^{\mathbf{P}}\left[e^{\lambda_{n}\left(\mu \Delta t+\sigma \epsilon_{n} \sqrt{\Delta t}\right)}\right]\right\} \\
& =\operatorname{argmax}_{\lambda_{n}}\left\{\lambda_{n}(r-\mu) \Delta t-\log \mathbf{E}^{\mathbf{P}}\left[e^{\lambda_{n} \sigma \epsilon_{n} \sqrt{\Delta t}}\right]\right\} \\
& =\operatorname{argmax}_{\lambda_{n}}\left\{\lambda_{n}(r-\mu) \Delta t-\log \mathbf{E}^{\mathbf{P}}\left[e^{\theta_{n}}\right]\right\}
\end{aligned}
$$

onde $\theta_{n} \sim \mathcal{N}\left(0, \lambda_{n}^{2} \sigma^{2} \Delta t\right)$, logo,

$$
\lambda_{n}^{*}=\operatorname{argmax}_{\lambda_{n}}\left\{\lambda_{n}(r-\mu) \Delta t-\frac{1}{2} \lambda_{n}^{2} \sigma^{2} \Delta t\right\}
$$


e, portanto,

$$
\lambda_{n}^{*}=-\frac{\mu-r}{\sigma^{2}} .
$$

É evidente pela fórmula acima que os $\lambda_{n}$ 's ótimos independem de $n$. Agora, introduzindo as equações (2.59) e (2.64) em (2.62), obtemos

$$
\begin{aligned}
\frac{d \pi_{n}}{d \mathbf{P}}(x) & =\frac{e^{-\frac{\mu-r}{\sigma^{2}}\left(\mu \Delta t+\sigma \epsilon_{n} \sqrt{\Delta t}\right)}}{\mathbf{E}^{\mathrm{P}}\left[e^{-\frac{\mu-r}{\sigma^{2}}\left(\mu \Delta t+\sigma \epsilon_{n} \sqrt{\Delta t}\right)}\right]} \\
& =\frac{e^{-\frac{\mu-r}{\sigma^{2}}\left(\mu \Delta t+\sigma \epsilon_{n} \sqrt{\Delta t}\right)}}{e^{-\frac{\mu-r}{\sigma^{2}} \mu \Delta t} \mathbf{E}^{\mathrm{P}}\left[e^{-\frac{\mu-r}{\sigma^{2}} \sigma \epsilon_{n} \sqrt{\Delta t}}\right]} \\
& =\frac{e^{-\frac{\mu-r}{\sigma} \epsilon_{n} \sqrt{\Delta t}}}{\mathbf{E}^{\mathrm{P}}\left[e^{-\frac{\mu-r}{\sigma} \epsilon_{n} \sqrt{\Delta t}}\right]}
\end{aligned}
$$

que é a medida de Arrow-Debreu em cada instante de tempo (discretizado). Então, a probabilidade $\pi$ de que uma determinada trajetória ocorra é o produto das $N$ medidas de probabilidades associadas a essas densidades. E assumindo que os retornos em cada instante sejam iid, a densidade dessa probabilidade "total" $\pi$ é dada por

$$
\frac{d \pi}{d \mathbf{P}}=\prod_{n=1}^{N} \frac{e^{-\frac{\mu-r}{\sigma} \epsilon_{n} \sqrt{\Delta t}}}{\mathrm{E}^{\mathbf{P}}\left[e^{-\frac{\mu-r}{\sigma} \epsilon_{n} \sqrt{\Delta t}}\right]}
$$

e, agora, tomando o logaritmo, temos

$$
\begin{aligned}
\log \frac{d \pi}{d \mathbf{P}} & =\log \prod_{n=1}^{N} \frac{e^{-\frac{\mu-r}{\sigma} \epsilon_{n} \sqrt{\Delta t}}}{\mathbf{E}^{\mathbf{P}}\left[e^{-\frac{\mu-r}{\sigma} \epsilon_{n} \sqrt{\Delta t}}\right]} \\
& =\sum_{n=1}^{N}\left(-\frac{\mu-r}{\sigma} \epsilon_{n} \sqrt{\Delta t}-\log \mathbf{E}^{\mathrm{P}}\left[e^{-\frac{\mu-r}{\sigma} \epsilon_{n} \sqrt{\Delta t}}\right]\right) \\
& =-\frac{\mu-r}{\sigma} \sqrt{\Delta t} \sum_{n=1}^{N} \epsilon_{n}-\log \mathbf{E}^{\mathbf{P}}\left[e^{-\frac{\mu-r}{\sigma} \epsilon_{n} \sqrt{\Delta t}}\right] \\
& =-\frac{\mu-r}{\sigma} \sqrt{\Delta t} \epsilon-\log \mathbf{E}^{\mathbf{P}}\left[e^{-\frac{\mu-r}{\sigma} \epsilon_{n} \sqrt{\Delta t}}\right]
\end{aligned}
$$

onde $\epsilon:=\sum_{n=1}^{N} \epsilon_{n} \sim \mathcal{N}(0, N)$ e, portanto,

$$
\log \frac{d \pi}{d \mathbf{P}}=-\frac{\mu-r}{\sigma} W_{T}-\frac{1}{2}\left(\frac{\mu-r}{\sigma}\right)^{2} T
$$

onde $W_{T} \sim \mathcal{N}(0, T)$ pois $N \Delta t=T$. 
Por outro lado, é sabido da teoria financeira que (2.66) é o logaritmo da densidade da medida de Arrow-Debreu usada para calcular a fórmula de Black e Scholes de apreçamento de opções como o valor presente esperado descontado pela taxa livre-de-risco do payoff de uma opção de compra. Com isso, demonstramos a compatibilidade do princípio da mínima entropia relativa com a teoria de Black e Scholes. Mais ainda, nós também expomos uma dedução mais simples da fórmula de Black e Scholes do que aquela usando unicamente cálculo estocástico.

\subsection{Entropia Relativa e a Informação de Fisher}

Ao assumirmos o Princípio da Mínima Entropia Relativa, a medida de ArrowDebreu que escolhemos tem a característica especial em relação às outras medidas de Arrow-Debreu admissíveis, isto é, em relação aquelas medidas de ArrowDebreu que satisfazem as restrições impostas, que é fato dela possuir a maior variância entre as mesmas. Contudo, essa é uma característica desejável, pois se o contrário acontecesse, estaríamos optando por uma medida que se concentrasse mais em torno de algum ponto e que, consequentemente, estivesse nos "dizendo" que a ocorrência de um evento na região em torno desse ponto é mais provável que eventos que em outras regiões. Porém, não há nenhuma informação nas restrições que nos leve a tal conclusão, de maneira que ao optarmos por uma medida de Arrow-Debreu de menor variância estamos considerando informações das quais não dispomos. Portanto, ao final das próximas seções teremos demonstrado o seguinte resultado:

Proposição 5 Se vale o Princípio da Mínima Entropia Relativa, então, entre todas medidas medidas de Arrow-Debreu que satisfazem um conjunto de restrições dadas nós escolhemos a de máxima variância.

\subsubsection{A Desigualdade de Cramér-Rao}

\section{Experimentos Estatísticos: uma Digressão}

Estatisticamente, nós interpretamos os resultados de observações com variáveis aleatórias $X_{1}, \ldots, X_{n}$, então, uma observação global é um vetor aleatório $X$ n-dimensional. Em geral, uma observação é uma variável aleatória $X$ assumindo valores no espaço mensurável $(\Xi, \mathcal{U})$ o qual denominamos por espaço amostral. Mais precisamente, existe um espaço de probabilidade $(\Omega, \mathcal{F}, \mathrm{P})$ e $X(\Omega, \mathcal{F}): \rightarrow(\Xi, \mathcal{U})$ é uma aplicação mensurável. Seja $\mathbf{P}^{X}(A):=\mathbf{P}(X \in A)$ a função distribuição de probabilidade de $X$ definida em $\mathcal{U}$. Em problemas estatísticos a única coisa que sabemos sobre a distribuição $\mathrm{P}^{X}$ é que ela pertence a uma classe de distribuições $\mathbf{P}$ e o conhecimento (baseado na observação $X$ ) dessa classe é um requisito da teoria. Por meio de uma escolha conveniente de um parâmetro, a família de distribuições $\mathbf{P}$ pode ser sempre parametrizada $\mathbf{e}$ representada da forma $\mathbf{P}=\left\{P_{\theta}, \theta \in \Theta\right\}$. 
Uma trinca arbitrária $\mathcal{E}:=\left\{\Xi, \mathcal{U},\left\{P_{\theta}, \theta \in \Theta\right\}\right\}$ chama-se experimento estatístico. Se um experimento estatístico é construido por meio da observação $X$, dizemos que o experimento é gerado pela observação $X$. Assumiremos que diferentes $P_{\theta^{\prime}}$ e $P_{\theta^{\prime \prime}}$ sobre $\mathcal{U}$ correspondem a diferentes $\theta^{\prime}$ e $\theta^{\prime \prime}$ em $\Theta$. Logo, vamos assumir que uma $\sigma$-álgebra $\mathcal{B}$ sobre $\Theta$ está definida.

Dizemos que uma medida $\sigma$-finita $\nu$ domina as distribuições $P_{\theta}, \theta \in \Theta$, se $P_{\theta} \ll \nu$ para todo $\theta \in \Theta$ e usamos a notação

$$
p(x, \theta):=\frac{d P_{\theta}}{d \nu}(x)
$$

e, portanto

$$
P_{\theta}(A)=\int_{A} p(x, \theta) d \nu(x)
$$

para todo $A \in \mathcal{U}$. Consideraremos apenas experimentos que possuem uma medida dominante. Assumiremos também que $p(x, \theta)$ é uma função mensurável (em relação ao par $(x, \theta)$ ).

Seja $(\Upsilon, \mathcal{L})$ um espaço mensurável. Uma estatística $T$ gerada por uma observação $X$ com valores em $(\Upsilon, \mathcal{L})$ é, por definição, qualquer função mensurável $T(x)$ a valores em $\Upsilon$. Se $\mathcal{E}=\left\{\Xi, \mathcal{U}, P_{\theta}, \theta \in \Theta\right\}$ é um experimento estatístico, uma estatística $T$ sobre $\mathcal{E}$ é, por definição, uma função mensurável $T:(\Xi, \mathcal{U}) \rightarrow(\Upsilon, \mathcal{L})$. A coleção de todas estatísticas sobre um dado experimento $\mathcal{E}$ contém toda a informação que pode ser extraída desse mesmo experimento.

Ainda considerando o experimento estatístico $\left\{\Xi, \mathcal{U}, P_{\theta}, \theta \in \Theta\right\}$ gerado pela observação $X$, seja $\varphi:(\Theta, \mathcal{B}) \rightarrow(\Upsilon, \mathcal{L})$ uma função mensurável e consideremos o problema de estimar o valor de $\varphi(\theta)$ no ponto $\theta$ baseados apenas na observação $X$, cuja distribuição é $P_{\theta}$. Nossa única informação é que $\theta \in \Theta$. Como estimador para $\varphi(\theta)$ podemos escolher qualquer função $T(X)$ com valores em $(\Upsilon, \mathcal{L})$. Um estimador de $\varphi(\theta)$ é não-enviesado se $\mathbf{E}^{\theta}[T]=\varphi(\theta)$ para todo $\theta \in \Theta$.

\section{Informação de Fisher e Experimentos Regulares}

Consideremos o experimento estatístico $\mathcal{E}=\left\{\Xi, \mathcal{U}, P_{\theta}, \theta \in \Theta\right\}$ onde $\Theta \in \mathbb{R}^{k}$ é aberto e $\nu$ uma medida dominante sobre $\mathcal{U}$. Como antes, denotemos $\frac{d P_{\theta}}{d \nu}$ por $p(x ; \theta)$. Suponhamos também que $p(x ; \theta)$ é uma função contínua de $\theta$ em $\Theta \mathrm{e}$ que é diferenciável no ponto $u=\theta$ para $\nu$-quase todo ponto $x$. Além disso, vamos assumir que

$$
g_{j j}(\theta ; \mathcal{E})=g_{j j}(\theta):=\int\left|\frac{\partial p(x ; \theta)}{\partial \theta_{j}}\right|^{2} \frac{d \nu(x)}{p(x ; \theta)}
$$

onde a integração é realizada sobre $\{x \in \Xi: p(x ; \theta) \neq 0$. Ou seja,

$$
g_{j j}(\theta)=\mathbf{E}^{\theta}\left[\left(\frac{\partial p(x ; \theta)}{\partial \theta_{j}} \frac{1}{p(x ; \theta)}\right)^{2}\right] \text {. }
$$


A desigualdade de Cauchy-Schwarz implica que as integrais

$$
g_{i j}(\theta):=\int \frac{\partial p(x ; \theta)}{\partial \theta_{i}} \frac{\partial p(x ; \theta)}{\partial \theta_{j}} \frac{d \nu(x)}{p(x ; \theta)}
$$

para $i, j=1, \ldots, k$ são convergentes.

Definição 12 A matriz

$$
g(\theta):=\left(g_{i j}(\theta)\right)_{i, j}
$$

chama-se Matriz de Informação de Fisher.

Em geral, para construir uma teoria de experimentos com informação de Fisher finita é necessário impor algumas condições de diferenciabilidade sobre a família $\{p(x ; \theta)\}$. Nós usaremos a seguinte definição:

Definição $13 U m$ experimento estatístico $\mathcal{E}$ é regular em $\Theta$ se

(a) $p(x ; \theta)$ é uma função contínua em $\Theta$ para $\nu$-quase todo ponto;

(b) $\mathcal{E}$ possui informação de Fisher finita em cada ponto $\theta \in \Theta$;

(c) A função $\psi(., \theta)$ é contínua no espaço $L^{2}(\nu)$.

A função $\psi(., \theta)$ é definida por

$$
\psi(x, \theta):=\frac{\partial}{\partial \theta} p^{\frac{1}{2}}(x, \theta) .
$$

\section{A Desigualdade de Cramér-Rao}

Teorema $22{ }^{7}$ Seja $\mathcal{E}=\left\{\Xi, \mathcal{U}, P_{\theta}, \theta \in \Theta\right\}$ um experimento regular com matriz de informação $g(\theta)>0, \Theta \subset \mathbb{R}^{k}$. Se a estatística $T=\left(T_{1}, \ldots, T_{k}\right): \Xi \rightarrow \mathbb{R}^{k}$ é tal que o risco $\mathbf{E}^{u}|T-u|^{2}$ é limitado na vizinhança do ponto $\theta \in \Theta$, então o viés

$$
b(u):=\mathbf{E}^{u}[T]-u
$$

é continuamente diferenciável em uma vizinhança de $\theta$ e a seguinte desigualdade matricial é satisfeita

$$
\mathbf{E}^{\theta}\left[(T-\theta)(T-\theta)^{T}\right] \geq\left(I+\frac{\partial}{\partial \theta} b(\theta)\right) g^{-1}(\theta)\left(I+\frac{\partial}{\partial \theta} b(\theta)\right)^{T}+b(\theta) b(\theta)^{T}
$$

onde I é a matriz identidade. Em particular, se $\Theta \subset \mathbb{R}$ e $g(\theta)>0$, então

$$
\mathrm{E}^{\theta}|T-\theta|^{2} \geq \frac{\left(1+b^{\prime}(\theta)\right)^{2}}{g(\theta)}+b^{2}(\theta) .
$$

\footnotetext{
${ }^{7}$ Para a demonstração desse teorema, veja [10].
} 
Se $T$ é uma estatística não-enviesada, isto é, se $b(u)=0$, então, as desigualdades acima podem ser reescritas da forma

$$
\operatorname{Var}(T) \geq g^{-1}(\theta) .
$$

Então, nesse caso a informação de Fisher fornece um limitante inferior para a variância dos estimadores de um parâmetro. Notemos que para estimar $\theta$ de modo que obtenhamos um estimador de mínima variância basta determinamos $\theta$ de modo a maximizarmos a informação de Fisher.

\subsubsection{Entropia Relativa e a Informação de Fisher}

Suponhamos agora que a medida dominante seja a medida de Lebesgue e que a família de distribuições admissíveis seja representada por uma família de funções densidade de probabilidade $\left(f_{\theta}\right)$, onde $\theta$ pertence a algum subconjunto $\Theta$ de $\mathbb{R}^{n}$. De (1.34) segue que:

$$
D\left(f_{\theta_{1}}, f_{\theta_{2}}\right)=\int f_{\theta_{1}} \log \left(\frac{f_{\theta_{1}}}{f_{\theta_{2}}}\right) d x .
$$

Fixemos $\theta$ e tomemos $\Delta \theta=\left(\Delta \theta_{1}, \ldots, \Delta \theta_{n}\right)$ um incremento em $\mathbb{R}^{n}$ a partir do qual definimos a seguinte função (de $\Delta \theta)$ :

$$
D_{\theta, \theta+\Delta \theta}:=D\left(f_{\theta}, f_{\theta+\Delta \theta}\right) .
$$

Então, definido $\Delta \log f_{\theta}=\log f_{\theta+\Delta \theta}-\log f_{\theta}$ como a variação em $\theta$ obtemos a seguinte fórmula:

$$
D_{\theta, \theta+\Delta \theta}=-\int f_{\theta} \Delta \log f_{\theta} d x
$$

Vamos supor agora que as distribuições $f_{\theta}$ satisfazem as seguintes condições de regularidade:

1. Para $\lambda$-quase todo ponto $x$, onde $\lambda$ é a medida de Lebesgue em $\mathbb{R}^{n}$, as derivadas parciais $\frac{\partial \log f_{\theta}}{\partial \theta_{i}}, \frac{\partial^{2} \log f}{\partial \theta_{i} \partial \theta_{j}}, \frac{\partial^{3} \log f}{\partial \theta_{i} \partial \theta_{j} \partial \theta_{l}}$ existem para todos $i, j, l \in$ $\{1 \ldots n\}$ para todo $\theta^{\prime}$ tal que $\theta<\theta^{\prime}<\theta+\Delta \theta$ (as desigualdades sendo consideradas componente a componente);

2. para todo $\theta^{\prime}$ nas condições acima, valem as desigualdades: $\left|\frac{\partial f(x)}{\partial \theta_{i}}\right|<F(x)$, $\left|\frac{\partial^{2} f(x)}{\partial \theta_{i} \partial \theta_{j}}\right|<G(x)$ e $\left|\frac{\partial^{3} f}{\partial \theta_{i} \partial \theta_{j} \partial \theta_{l}}\right|<H(x)$ para todos $i, j, l \in\{1, \ldots, n\}$, onde $F(x), G(x)$ são integráveis e existe $M>0$ tal que $\int f_{\theta}(x) H(x) d x<M<$ $\infty$;

3. $\int \frac{\partial f}{\partial \theta_{i}} d x=0, \int \frac{\partial^{2} f}{\partial \theta_{i} \partial \theta_{j}} d x=0$, para todos $i, j \in\{1, \ldots, n\}$; 
e, então, expandir $\Delta \log f_{\theta}$ em série de Taylor em torno de $\theta$ :

$$
\begin{aligned}
\log f_{\theta+\Delta \theta}= & \log f_{\theta}+\frac{1}{2} \sum_{i=1}^{n} \frac{\partial \log f_{\theta}}{\partial \theta_{i}} \Delta \theta_{i}+\sum_{i=1}^{n} \sum_{j=1}^{n} \frac{\partial^{2} \log f_{\theta}}{\partial \theta_{i} \partial \theta_{j}} \Delta \theta_{i} \Delta \theta_{j} \\
& +\sum_{i=1}^{n} \sum_{j=1}^{n} \sum_{l=1}^{n}\left[\frac{\partial^{3} \log f_{\theta}}{\partial \theta_{i} \partial \theta_{j} \partial \theta_{l}}\right]_{\theta+t \Delta \theta} \Delta \theta_{i} \Delta \theta_{j} \Delta \theta_{l}
\end{aligned}
$$

para algum $t \in[0,1]^{n}$. Logo, calculando as derivadas de primeira e segunda ordens e subtraindo ambos os lados da equação acima por $\log f_{\theta}$ obtemos:

$$
\begin{aligned}
\Delta \log f_{\theta}= & \log f_{\theta+\Delta \theta}-\log f_{\theta}=\frac{1}{2} \sum_{i=1}^{n} \frac{1}{f_{\theta}} \frac{\partial f_{\theta}}{\partial \theta_{i}} \Delta \theta_{i} \\
& +\sum_{i, j=1}^{n}\left(\frac{1}{f_{\theta}} \frac{\partial^{2} f_{\theta}}{\partial \theta_{i} \partial \theta_{j}}-\frac{1}{f_{\theta}^{2}} \frac{\partial f_{\theta}}{\partial \theta_{i}} \frac{\partial f_{\theta}}{\partial \theta_{j}}\right) \Delta \theta_{i} \Delta \theta_{j} \\
& +\sum_{i, j, l=1}^{n}\left[\frac{\partial^{3} \log f_{\theta}}{\partial \theta_{i} \partial \theta_{j} \partial \theta_{l}}\right]_{\theta+t \Delta \theta} \Delta \theta_{i} \Delta \theta_{j} \Delta \theta_{l}
\end{aligned}
$$

para algum $t \in[0,1]^{n}$. Logo, pelas condições de regularidade dadas acima:

$$
\begin{aligned}
D_{\theta, \theta+\Delta \theta}= & -\frac{1}{2} \sum_{i=1}^{n} \Delta \theta_{i} \int \frac{\partial f_{\theta}}{\partial \theta_{i}} d x \\
& -\sum_{i, j=1}^{n} \Delta \theta_{i} \Delta \theta_{j} \int\left(\frac{\partial^{2} f_{\theta}}{\partial \theta_{i} \partial \theta_{j}}-\frac{1}{f_{\theta}} \frac{\partial f_{\theta}}{\partial \theta_{i}} \frac{\partial f_{\theta}}{\partial \theta_{j}}\right) d x \\
& -\sum_{i, j, l=1}^{n} \Delta \theta_{i} \Delta \theta_{j} \Delta \theta_{l} \int\left[\frac{\partial^{3} \log f_{\theta}}{\partial \theta_{i} \partial \theta_{j} \partial \theta_{l}}\right]_{\theta+t \Delta \theta} d x \\
= & \sum_{i, j=1}^{n} \Delta \theta_{i} \Delta \theta_{j} \int \frac{1}{f_{\theta}} \frac{\partial f_{\theta}}{\partial \theta_{i}} \frac{\partial f_{\theta}}{\partial \theta_{j}} d x \\
& -\sum_{i, j, l=1}^{n} \Delta \theta_{i} \Delta \theta_{j} \Delta \theta_{l} \int\left[\frac{\partial^{3} \log f_{\theta}}{\partial \theta_{i} \partial \theta_{j} \partial \theta_{l}}\right]_{\theta+t \Delta \theta} d x
\end{aligned}
$$

para algum $t \in[0,1]^{n}$. E como pela segunda condição de regularidade o termo de terceira ordem da soma acima pode tornar-se arbitrariamente pequeno conforme diminuimos o tamanho dos incrementos $\Delta \theta_{i}, \Delta \theta_{j}$ e $\Delta \theta_{l}$, então $D_{\theta, \theta+\Delta \theta}$ pode 
ser aproximada com um grau de precisão arbitrário por:

$$
\begin{aligned}
D_{\theta+\Delta \theta} & =\sum_{i, j=1}^{n} \Delta \theta_{i} \Delta \theta_{j} \int \frac{1}{f_{\theta}} \frac{\partial f_{\theta}}{\partial \theta_{i}} \frac{\partial f_{\theta}}{\partial \theta_{j}} d x+O\left((\Delta \theta)^{3}\right) \\
& =\sum_{i, j=1}^{n} \Delta \theta_{i} \Delta \theta_{j} \int f_{\theta}\left(\frac{1}{f_{\theta}} \frac{\partial f_{\theta}}{\partial \theta_{i}}\right)\left(\frac{1}{f_{\theta}} \frac{\partial f_{\theta}}{\partial \theta_{j}}\right) d x+O\left((\Delta \theta)^{3}\right) \\
& =\sum_{i, j=1}^{n} g_{i j} \Delta \theta_{i} \Delta \theta_{j}+O\left((\Delta \theta)^{3}\right)
\end{aligned}
$$

onde $\left(g_{i j}\right)_{i j}=\left(\int f_{\theta}\left(\frac{1}{f_{\theta}} \frac{\partial f_{\theta}}{\partial \theta_{i}}\right)\left(\frac{1}{f_{\theta}} \frac{\partial f_{\theta}}{\partial \theta_{j}}\right) d x\right)_{i j}$ é a Matriz de Informação de Fisher.

Essa relação entre a entropia relativa e a Informação de Fisher reforça ainda mais o caráter métrico da entropia relativa. De fato, como podè-se observar a partir do trabalho de Amari [1], pode-se lidar com os espaços das distribuições de probabilidade com um enfoque geométrico. Mais precisamente, ele demonstra serem esses espaços variedades riemannianas. O ponto interessante aqui é que nesses espaços a métrica de Riemann por ele determinada é exatamente a Matriz de Informação de Fisher. Desse modo, quanto menor a entropia relativa entre duas distrubuições, menor a distância entre elas nessas variedades. 


\section{Capítulo 3}

\section{Modelos Intertemporais}

\subsection{Calibrando uma Superfície de Volatilidade}

Nesta seção utilizaremos o princípio da entropia relativa para modelar uma superfície de volatilidade. O processo consiste em determinar um processo difusivo livre de arbitragem que minimize a entropia relativa em relação a uma difusão dada a priori. Ao modelarmos o problema em questão, mostraremos no que necessitamos resolver um problema de controle ótimo com restrições. A resolução desse problema de otimização baseia-se no Princípio de Programação Dinâmica de Bellman. Portanto, determinaremos a EDP de Hamilton-Jacobi-Bellman associada a esse problema de otimização, mostraremos a existência e unicidade de solução para essa EDP e, finalmente, como extrair a superfície de volatilidade a partir dessa solução.

\subsubsection{Aproximando a Entropia Relativa via Árvores Tri- nomiais}

Sejam $\mathbf{P}$ e $\mathbf{Q}$ duas medidas de probabilidade (a primeira delas representa uma medida dada a priori, inferida diretamente no mercado via procedimentos econométricos) e suponha que ambas estão definidas sobre o conjunto $\Omega:=C[0, T]$, satisfazendo:

$$
\frac{d S_{t}^{(1)}}{S_{t}^{(1)}}=\sigma_{t}^{(1)} d W_{t}+\mu_{t}^{(1)} d t
$$

e

$$
\frac{d S_{t}^{(2)}}{S_{t}^{(2)}}=\sigma_{t}^{(2)} d W_{t}+\mu_{t}^{(2)} d t
$$

no sentido de Itô. Aqui, dado $\omega \in \Omega, W_{t}(\omega):=\omega(t)$ é um movimento Browniano com relação à medida de probabilidade estendida, através do teorema de 
extensão de Kolmogorov, a partir das pró-medidas Gaussianas definidas sobre cilindros do tipo:

$$
C\left(t_{1}, \ldots, t_{n} ; I_{1}, \ldots, I_{n}\right):=\left\{\omega:\left(\omega\left(t_{1}\right), \ldots, \omega\left(t_{n}\right)\right) \in I_{1} \times \ldots \times I_{n}\right\}
$$

onde $I_{1}, \ldots, I_{n}$ são intervalos na reta.

Dados $n \in \mathbb{N}, B_{1}, \ldots, B_{n} \in \mathcal{B}(\mathbb{R})$ e $t_{1}, \ldots, t_{n} \in[0, T]$ sejam:

$$
\mathbf{P}_{t_{1}, \ldots, t_{n}}\left(B_{1}, \ldots, B_{n}\right):=\mathbb{W}\left(S_{t_{1}}^{(1)} \in B_{1}, \ldots, S_{t_{n}}^{(1)} \in B_{n}\right)
$$

e analogamente,

$$
\mathrm{Q}_{t_{1}, \ldots, t_{m}}\left(B_{1}, \ldots, B_{n}\right):=\mathbb{W}\left(S_{t_{1}}^{(2)} \in B_{1}, \ldots, S_{t_{n}}^{(2)} \in B_{n}\right)
$$

definidas em $\mathcal{B}\left(\mathbb{R}^{n}\right)$. W é a medida de Wiener sobre $\Omega$. Estamos assumindo os processos $\sigma^{\star}$ e $\lambda^{\star}$ limitadas e progressivamente mensuráveis. Afirmamos que $\mathbf{P}_{t_{1}, \ldots, t_{n}}$ e $\mathbf{Q}_{t_{1}, \ldots, t_{n}}$ são, de fato, medidas de probabilidade, mais ainda, são pró-medidas que satisfazem o critério de consistência de Kolmogorov:

"Uma família de medidas de probabilidade $\left\{\mathbf{P}_{u}: u \subset \tau,|u|<\infty\right\}$ onde cada $\mathbf{P}_{u}$ é uma medida sobre $\left(\mathbb{R}^{|u|}, \mathcal{B}\left(\mathbb{R}^{|u|}\right)\right)$ é dita consistente no sentido de Kolmogorov se

$$
\mathbf{P}_{u}\left(\pi_{u, v}^{-1}(B)\right)=\mathbf{P}_{v}(B)
$$

para todo $v \subset$ u e $B \in \mathcal{B}\left(\mathbb{R}^{|v|}\right)$."

obs. 5

A função $\pi_{u, v}(B)$ é definida da seguinte maneira: seja $\tau$ um conjunto qualquer (em geral, $\tau \subset \mathbb{N}$ ou $\tau$ é um intervalo na reta) e $u=\left\{t_{1}, \ldots, t_{n}\right\} \subset \tau$ satisfazendo $u \neq \emptyset$ e $|u|=n<\infty$. Para $v=\left\{t_{i_{1}}, \ldots, t_{i_{k}}\right\} \in u$ tal que $\left\{i_{1}, \ldots, i_{k}\right\} \subset\{1, \ldots, n\}$ definimos

$$
\begin{aligned}
\pi_{u, v}: \mathbb{R}^{|u|} & \rightarrow \mathbb{R}^{|v|} \\
\left(x_{1}, \ldots, x_{n}\right) & \mapsto\left(x_{i_{1}}, \ldots, x_{i_{k}}\right)
\end{aligned}
$$

De fato,

1. $\mathbf{P}_{t_{1}, \ldots, t_{n}}\left(\mathbb{R}^{n}\right)=1$.

Esta propriedade é trivial, pois para cada $\omega$ temos que $S_{t}(\omega)$ é uma função real contínua, logo, $S_{t_{1}}^{(1)^{-1}}(\mathbb{R}) \cap \ldots \cap S_{t_{n}}^{(1)^{-1}}(\mathbb{R})=\Omega$ e, portanto, $\mathbf{P}_{t_{1}, \ldots, t_{n}}\left(\mathbb{R}^{n}\right)=\mathbb{W}(\Omega)=1$.

2. $\mathbf{P}_{t_{1}, \ldots, t_{n}} \geq 0$.

Imediato, uma vez que $\mathbb{W} \geq 0$. 
3. $\mathbf{P}_{t_{1}, \ldots, t_{n}}\left(\dot{\cup}_{j=1}^{\infty} B_{1, j} \times \ldots \times B_{n, j}\right)=\sum_{j=1}^{\infty} \mathbf{P}_{t_{1}, \ldots, t_{n}}\left(B_{1, j} \times \ldots \times B_{n, j}\right)$.

De fato, dado $j \in \mathbb{N}$ consideremos conjuntos $B_{j} \in \mathcal{B}\left(\mathbb{R}^{n}\right)$ dois a dois disjuntos. Então,

$$
\begin{aligned}
\mathbf{P}_{t_{1}, \ldots, t_{n}}\left(\bigcup_{j=1}^{\infty} B_{j}\right) & =\mathbb{W}\left(\left(S_{t_{1}}^{(1)}, \ldots, S_{t_{n}}^{(1)}\right) \in \bigcup_{j=1}^{\infty} B_{j}\right) \\
& =\mathbb{W}\left(\bigcup_{j=1}^{\infty}\left\{\omega:\left(S_{t_{1}}^{(1)}(\omega), \ldots, S_{t_{n}}^{(1)}(\omega)\right) \in B_{j}\right\}\right) \\
& =\sum_{j=1}^{\infty} \mathbb{W}\left(\left\{\omega:\left(S_{t_{1}}^{(1)}(\omega), \ldots, S_{t_{n}}^{(1)}(\omega)\right) \in B_{j}\right\}\right) \\
& =\sum_{j=1}^{\infty} \mathbf{P}_{t_{1}, \ldots, t_{n}}\left(B_{j}\right)
\end{aligned}
$$

4. $\left\{\mathbf{P}_{t_{1}, \ldots, t_{n}}: t_{1}, \ldots, t_{n} \in[0, T], n \in \mathbb{N}\right\}$ é uma família de medidas de probabilidade consistente no sentido de Kolmogorov.

De fato, sejam $u=\left\{t_{1}, \ldots, t_{n}\right\}, v=\left\{t_{i_{1}}, \ldots, t_{i_{n}}\right\} \subset u$, e $B \in \mathcal{B}\left\{\mathbb{R}^{|v|}\right)$ quaisquer. Então,

$$
\begin{aligned}
\mathbf{P}_{v}(B) & =\mathbb{W}\left(\left(S_{t_{i_{1}}}, \ldots, S_{t_{i_{k}}}\right) \in B\right) \\
& =\mathbb{W}\left(\left(S_{t_{1}}, \ldots, S_{t_{n}}\right) \in \pi_{u, v}^{-1}(B)\right) \\
& =\mathbf{P}_{u}\left(\pi_{u, v}^{-1}(B)\right)
\end{aligned}
$$

pois $\mathbb{W}$ é por definição a extensão através do teorema de Kolmogorov de pró-medidas consistentes.

Analogamente, as propriedades (1), (2), (3) e (4) são verdadeiras para o conjunto de medidas

$$
\left\{\mathrm{Q}_{t_{1}, \ldots, t_{n}}: n \in \mathbb{N} ; t_{1}, \ldots, t_{n} \in[0, T)\right\} .
$$

Agora, aplicando o teorema de extensão de Kolmogorov

"Se $\left\{\mathbf{P}_{u}: u \subset \tau,|u|<\infty\right\}$ é um conjunto consistente de medidas de probabilidade sobre $\mathcal{B}\left(\mathbb{R}^{|u|}\right)$, então, existe uma única medida de probabilidade $\mathbf{P}$ em $\mathcal{C}\left(\mathbb{R}^{\top}\right)$ que satisfaz

$$
\mathbf{P}(C(u ; B))=\mathbf{P}_{u}(B)
$$

para todo $u=\left\{t_{1}, \ldots, t_{n}\right\} \subset \tau,|u|=n<\infty$ e $B \in \mathcal{B}\left(\mathbb{R}^{|u|}\right)$."

temos a existência e unicidade de medidas de probabilidade $\mathbf{P}$ e $\mathbf{Q}$ associadas aos processos de preços $S_{t}^{(1)}$ e $S_{t}^{(2)}$, respectivamente, e definidas na $\sigma$-álgebra gerada pelos cilindros $C\left(t_{1}, \ldots, t_{n} ; I_{1}, \ldots, I_{n}\right)$, onde $I_{1}, \ldots, I_{n}$ são intervalos na reta. 
Note que utilizando essas probabilidades, um cilindro $C\left(t_{1}, \ldots, t_{n} ; I_{1}, \ldots, I_{n}\right)$ tem medida $\mathbf{P}$ (ou $\mathbf{Q}$ ) positiva se, e somente se, o conjunto de trajetórias $\omega$ que satisfazem a equação (3.1). (ou (3.2)) e que esteja contido neste cilindro também tem medida $\mathbf{P}$ (ou $\mathbf{Q}$ ) positiva. A intenção agora é calcular $D(\mathbf{P}, \mathbf{Q})$ para estas medidas. No caso em que $\sigma^{(1)}=\sigma^{(2)}=\sigma$ o cálculo é mais simples e segue do teorema de Girsanov ${ }^{1}$

Proposição 6 Se $\sigma^{(1)}=\sigma^{(2)}=\sigma$ Q-q.s. nas equações (3.1) e (3.2), tem-se:

$$
D(\mathbf{P}, \mathbf{Q})=\frac{1}{2} \mathbf{E}^{\mathbf{Q}}\left\{\int_{0}^{T}\left(\frac{\mu_{t}^{\mathrm{Q}}-\mu_{t}^{\mathrm{P}}}{\sigma_{t}}\right)^{2} d t\right\}
$$

Prova. Consideremos os processos

$$
\frac{d S^{(1)}(t)}{S^{(1)}(t)}=\sigma(t) d W(t)+\mu^{(1)}(t) d t
$$

e

$$
\frac{d S^{(2)}(t)}{S^{(2)}(t)}=\sigma(t) d W(t)+\mu^{(2)}(t) d t .
$$

Seja $\mathbf{Q}$ a distribuição de $S^{(2)}(t)$ definida acima. Então, pelo Teorema de Girsanov, tomemos

$$
u(t):=\frac{\mu^{(2)}(t)-\mu^{(1)}(t)}{\sigma(t)}
$$

e definemos a medida $\mathbf{P}_{\mathbf{1}}$ por

$$
d \mathbf{P}_{1}:=e^{-\int_{0}^{T} u(s, \omega) d W(s)-\frac{1}{2} \int_{0}^{T} u^{2}(s, \omega) d s} d \mathbf{Q}
$$

isto é

$$
\frac{d \mathbf{Q}}{d \mathbf{P}_{1}}:=e^{\int_{0}^{T} u(s, \omega) d W(s)+\frac{1}{2} \int_{0}^{T} u^{2}(s, \omega) d s} .
$$

Então, temos que o processo $S^{(1)}(t)$ acima pode ser reescrito da forma

$$
\frac{d S^{(1)}(t)}{S^{(1)}(t)}=\sigma(t) d \hat{W}(t)+\mu^{(2)}(t) d t
$$

onde

$$
d \hat{W}(t):=u(t) d t+d W(t)
$$

é um movimento browniano com relação a distribuição $\mathbf{P}_{1}$. Mais ainda, segundo o Teorema de Girsanov, $\mathbf{P}_{1}$ é a distribuição do processo $S^{(1)}(t)$ no sentido que

$$
\mathbf{P}_{1}\left(B_{1}, \ldots, B_{n}\right)=\mathbb{W}\left(S_{t_{1}}^{(1)} \in B_{1}, \ldots, S_{t_{n}}^{(1)} \in B_{n}\right)
$$

${ }^{1}$ No caso em que $\sigma_{t}^{(1)} \neq \sigma_{t}^{(2)}$ temos que $\mathrm{P}$ e $\mathrm{Q}$ são mutuamente singulares e, consequentemente, a entropia relativa entre $\mathbf{P}$ e $Q$ é, por definição, igual a $+\infty$. 
onde $B_{1}, \ldots, B_{n} \in \mathcal{B}(\mathbb{R})$ e $t_{1}, \ldots, t_{n} \in[0, T]$, para todo $n \in \mathbb{N}$.

Por outro lado, usando o Teorema de Extensão de Kolmogorov, vimos que $\mathbf{P}$ é a única medida em $\mathcal{C}\left(\mathbb{R}^{\tau}\right)$ que satisfaz (3.5). Logo, $\mathbf{P}=\mathbf{P}_{1} \mathrm{e}$, portanto,

$$
\log \frac{d \mathbf{Q}}{d \mathbf{P}}:=\int_{0}^{T} u(s, \omega) d W(s)+\frac{1}{2} \int_{0}^{T} u^{2}(s, \omega) d s .
$$

Agora, tomando a esperança em relação a medida $\mathrm{Q}$

$$
\mathbf{E}^{\mathbf{Q}}\left[\log \frac{d \mathbf{Q}}{d \mathbf{P}}\right]=\frac{1}{2} \mathbf{E}^{\mathbf{Q}} \int_{0}^{T} u^{2}(s, \omega) d s
$$

ou seja,

$$
D(\mathbf{P}, \mathbf{Q})=\mathbf{E}^{\mathbf{Q}}\left[\log \frac{d \mathbf{Q}}{d \mathbf{P}}\right]=\frac{1}{2} \mathrm{E}^{\mathbf{Q}} \int_{0}^{T}\left(\frac{\mu^{(2)}(t)-\mu^{(1)}(t)}{\sigma(t)}\right)^{2} d s .
$$

Como não estamos interessados particularmente no caso em que as volatilidades são idênticas vamos considerar aproximações em tempo discreto e analisar o comportamento da sequência de entropias quando a grade tende a zero.

Para isso, consideremos duas medidas de probabilidade $\mathbf{P}$ e $\mathbf{Q}$ definidas sobre caminhos discretos

$$
S_{0}, S_{1}, \ldots, S_{N}
$$

onde $\mathrm{N}$ é algum inteiro. A P-probabilidade de que tal trajetória ocorra é dada por:

$$
\prod_{n=0}^{N-1} \pi_{n}^{\mathbf{P}}
$$

onde $\pi_{n}^{\mathrm{P}}$ é a probabilidade condicional de que o preço do ativo no instante $n+1$ seja $S_{n+1}$ dada a informação disponível até o tempo $n$, mais precisamente:

$$
\pi_{n}^{\mathrm{P}}:=\mathbf{P}\left(. \mid \mathcal{F}_{n}\right)
$$

onde $\mathcal{F}_{n}$ representa a $\sigma$-álgebra gerada pela partição do espaço de todos os caminhos possíveis, de modo que duas trajetórias pertencem ao mesmo elemento dessa partição se, e somente se, elas coincidem até o instante $n$. Uma notação análoga será usada para $\mathbf{Q}$. De (1.32), a entropia relativa de $\mathbf{Q}$ em relação à $\mathbf{P}$ 
é dada por:

$$
\begin{aligned}
D(\mathbf{P}, \mathbf{Q}) & =\sum_{\text {trajetórias }}\left(\prod_{n=0}^{N-1} \pi_{n}^{\mathbf{Q}}\right) \log \left(\frac{\prod_{n=0}^{N-1} \pi_{n}^{\mathbf{Q}}}{\prod_{n=0}^{N-1} \pi_{n}^{\mathbf{P}}}\right) \\
& =\mathbf{E}^{\mathbf{Q}}\left[\log \left(\prod_{n=0}^{N-1} \frac{\pi_{n}^{\mathbf{Q}}}{\pi_{n}^{\mathbf{P}}}\right)\right] \\
& =\mathbf{E}^{\mathbf{Q}}\left[\sum_{n=0}^{N-1} \log \frac{\pi_{n}^{\mathbf{Q}}}{\pi_{n}^{\mathbf{P}}}\right] \\
& =\mathbf{E}^{\mathbf{Q}}\left[\mathbf{E}_{n}^{\mathbf{Q}}\left[\sum_{n=0}^{N-1} \log \frac{\pi_{n}^{\mathbf{Q}}}{\pi_{n}^{\mathbf{P}}}\right]\right] \\
& =\mathbf{E}^{\mathbf{Q}}\left[\sum_{n=0}^{N-1} \mathbf{E}_{n}^{\mathbf{Q}}\left[\log \frac{\pi_{n}^{\mathbf{Q}}}{\pi_{n}^{\mathbf{P}}}\right]\right]
\end{aligned}
$$

Em (3.6) utilizamos a notação $E_{n}^{\mathbf{Q}}$ para denotar a esperança condicional dada a informação disponível até o tempo $n$ (ou seja, $E_{n}^{\mathbf{Q}}[]=.E^{\mathrm{Q}}\left[. \mid \mathcal{F}_{n}\right]$ ) com relação à medida $\mathrm{Q}$. A penúltima passagem justifica-se pela propriedade de que dados $X$, uma variável aleatória, e $\mathbf{S}$, uma medida de probabilidade, então $\mathbf{E}^{\mathbf{S}}[X]=$ $\mathbf{E}^{\mathbf{S}}\left\{\mathbf{E}^{\mathbf{S}}[X \mid \mathcal{F}]\right\}$, onde $\mathcal{F}$ é uma sub- $\sigma$-álgebra qualquer da $\sigma$-álgebra na qual a medida de probabilidade $\mathbf{S}$ está definida.

Em outras palavras, a entropia relativa entre $\mathbf{P}$ e $\mathbf{Q}$ é obtida somando-se as entropias relativas condicionais $\mathbf{E}_{n}^{\mathbf{Q}}\left[\log \left(\frac{\pi_{n}^{\mathbf{Q}}}{\pi_{n}^{P}}\right)\right]$, ao longo de cada percurso, e então calculando-se a média delas com relação a $\mathbf{Q}$ sobre todas trajetórias possíveis.

Aproximaremos os processos de Itô (3.1) e (3.2) de modo que a entropia possa ser calculada explicitamente quando $N$ tende ao infinito. Essas aproximações são realizadas via árvores trinomiais (a escolha por árvores trinomiais é por pura conveniência no momento em que formos resolver numericamente a equação diferencial parcial de Hamilton-Jacobi-Bellman pelo método de diferenças finitas). Desse modo, assumiremos que o processo de preços é dado por:

$$
S_{n+1}=S_{n} H_{n+1}
$$

com $n=0,1, \ldots, N$ e

$$
H_{n+1}= \begin{cases}e^{\zeta \sqrt{\Delta t}} & \text { com probabilidade } \mathbf{P}_{u} \\ 1 & \text { com probabilidade } \mathbf{P}_{m} \\ e^{-\zeta \sqrt{\Delta t}} & \text { com probabilidade } \mathbf{P}_{d}\end{cases}
$$

para $\zeta>0$. Aqui o índice $n$ representa a data $t_{n}=n \frac{T}{N}$ e $\Delta t$ o intervalo entre as datas, ou seja, $\Delta t=\frac{T}{N}$. As probabilidades de transição $P_{u}, P_{m}$ e $P_{d}$ são dadas 
por:

$$
\begin{aligned}
& \mathbf{P}_{u}=\frac{p}{2}\left(1-\frac{\zeta \sqrt{\Delta t}}{2}\right)+\frac{\mu \sqrt{\Delta t}}{2 \zeta} \\
& \mathbf{P}_{m}=1-p \\
& \mathbf{P}_{d}=\frac{p}{2}\left(1+\frac{\zeta \sqrt{\Delta t}}{2}\right)-\frac{\mu \sqrt{\Delta t}}{2 \zeta}
\end{aligned}
$$

onde $p$ é um parâmetro entre 0 e 1 . Note que o processo $\log S_{n}$ descreve um passeio aleatório no conjunto $\{k \zeta \sqrt{\Delta t}: k \in \mathbb{Z}\}$. Além disso, com as probabilidades definidas acima a média e a volatilidade do processo $\log S_{n}$ são consistentes com os processos de Itô (3.1) ou (3.2). De fato,

$$
\begin{aligned}
\mathbf{E}\left[\log S_{n+1}\right] & =\log \left(S_{n} e^{\zeta \sqrt{\Delta t}}\right) \mathbf{P}_{u}+\log \left(S_{n}\right) \mathbf{P}_{m}+\log \left(S_{n} e^{-\zeta \sqrt{\Delta t}}\right) \mathbf{P}_{d} \\
& =\log S_{n}+\zeta \sqrt{\Delta t}\left(\mathbf{P}_{u}-\mathbf{P}_{d}\right) \\
& =\log S_{n}+\left(\mu-\frac{p \cdot \zeta^{2}}{2}\right) \Delta t
\end{aligned}
$$

ou seja, a média instantânea é igual a $\mu-\frac{p \cdot \zeta^{2}}{2}$. Analogamente, mostra-se que a variância instantânea de $\log S_{n}$ é igual a $p \cdot \zeta^{2}$. Interpretamos $\mu$ como o custo de carregamento e $\zeta \sqrt{p}$ como a volatilidade do índice. Esse modelo acomoda, variando-se o parâmetro $p$, processos com volatilidade variável dentro do intervalo $(0, \zeta]$

Denotaremos os parâmetros referentes às duas medidas $\mathbf{P}$ e $\mathbf{Q}$ por $(p, \mu) \mathrm{e}$ $(q, \nu)$, respectivamente. Então,

$$
\begin{aligned}
\mathbf{E}_{n}^{\mathbf{Q}}\left[\log \frac{\pi_{n}^{\mathrm{Q}}}{\pi_{n}^{\mathrm{P}}}\right] & =\left[\frac{q}{2}\left(1-\frac{\zeta \sqrt{\Delta t}}{2}\right)+\frac{\nu \sqrt{\Delta t}}{2 \zeta}\right] \log \frac{\frac{q}{2}\left(1-\frac{\zeta \sqrt{\Delta t}}{2}\right)+\frac{\nu \sqrt{\Delta t}}{2 \zeta}}{\frac{p}{2}\left(1-\frac{\zeta \sqrt{\Delta t}}{2}\right)+\frac{\mu \sqrt{\Delta t}}{2 \zeta}} \\
& +(1-q) \log \frac{1-q}{1-p} \\
& +\left[\frac{q}{2}\left(1+\frac{\zeta \sqrt{\Delta t}}{2}\right)-\frac{\nu \sqrt{\Delta t}}{2 \zeta}\right] \log \frac{\frac{q}{2}\left(1+\frac{\zeta \sqrt{\Delta t}}{2}\right)-\frac{\nu \sqrt{\Delta t}}{2 \zeta}}{\frac{p}{2}\left(1+\frac{\zeta \sqrt{\Delta t}}{2}\right)-\frac{\mu \sqrt{\Delta t}}{2 \zeta}}
\end{aligned}
$$

Comecemos, então, analisando o termo

$$
\log \frac{\frac{q}{2}\left(1-\frac{\zeta \sqrt{\Delta t}}{2}\right)+\frac{\nu \sqrt{\Delta t}}{2 \zeta}}{\frac{p}{2}\left(1-\frac{\zeta \sqrt{\Delta t}}{2}\right)+\frac{\mu \sqrt{\Delta t}}{2 \zeta}}:=L_{1} .
$$


Temos que

$$
\begin{aligned}
\frac{q}{2}\left(1-\frac{\zeta \sqrt{\Delta t}}{2}\right)+\frac{\nu \sqrt{\Delta t}}{2 \zeta} & =\frac{q}{2}+\left(\frac{\nu}{2 \zeta}-\frac{q \zeta}{4}\right) \sqrt{\Delta t} \\
& =\frac{q}{2}\left[1+\frac{2}{q}\left(\frac{\nu}{2 \zeta}-\frac{q \zeta}{4}\right) \sqrt{\Delta t}\right] \\
& =\frac{q}{2}(1+\alpha \sqrt{\Delta t})
\end{aligned}
$$

onde $\alpha=\frac{\nu}{q \zeta}-\frac{\zeta}{2} \mathrm{e}$, analogamente,

$$
\frac{p}{2}\left(1-\frac{\zeta \sqrt{\Delta t}}{2}\right)+\frac{\mu \sqrt{\Delta t}}{2 \zeta}=\frac{p}{2}(1+\beta \sqrt{\Delta t})
$$

onde $\beta=\frac{\mu}{p \zeta}-\frac{\zeta}{2}$. Portanto,

$$
L_{1}=\log \frac{q}{p}+\log (1+\alpha \sqrt{\Delta t})-(1+\beta \sqrt{\Delta t}) .
$$

Agora, usando a expansão em série de Taylor

$$
\log (1+h)=h+o(h)
$$

para $h$ suficientemente pequeno, obtemos

$$
\log (1+\alpha \sqrt{\Delta t})=\alpha \sqrt{\Delta t}+o(\Delta t)
$$

$$
\log (1+\beta \sqrt{\Delta t})=\beta \sqrt{\Delta t}+o(\Delta t)
$$

onde tomamos $h=\alpha \sqrt{\Delta t}$ e $h=\beta \sqrt{\Delta t}$, respectivamente. Então, substituindo (3.10) e (3.11) em (3.9)

$$
L_{1}=\log \frac{q}{p}+(\alpha-\beta) \sqrt{\Delta t}
$$

ou seja,

$$
L_{1}=\log \frac{q}{p}+\frac{1}{\zeta}\left(\frac{\nu}{q}-\frac{\mu}{p}\right) \sqrt{\Delta t} .
$$

De modo exatamente análogo, obtemos a igualdade

$$
\log \left(\frac{\frac{q}{2}\left(1+\frac{\zeta \sqrt{\Delta t}}{2}\right)-\frac{\nu \sqrt{\Delta t}}{2 \zeta}}{\frac{q}{2}\left(1+\frac{\zeta \sqrt{\Delta t}}{2}\right)-\frac{\nu \sqrt{\Delta t}}{2 \zeta}}\right)=\log \frac{\dot{q}}{p}+\frac{1}{\zeta}\left(\frac{\mu}{p}-\frac{\nu}{q}\right) \sqrt{\Delta t}+o(\Delta t)
$$


Então, substituindo (3.12) e (3.13) em (3.8), temos

$$
\begin{aligned}
\mathbf{E}_{n}^{\mathrm{Q}}\left[\log \frac{\pi_{n}^{\mathrm{Q}}}{\pi_{n}^{\mathrm{P}}}\right] & =\left[\frac{q}{2}\left(1-\frac{\zeta \sqrt{\Delta t}}{2}\right)+\frac{\nu \sqrt{\Delta t}}{2 \zeta}\right]\left[\log \frac{q}{p}+\frac{1}{\zeta}\left(\frac{\nu}{q}-\frac{\mu}{p}\right) \sqrt{\Delta t}+o(\Delta t)\right] \\
& +(1-q) \frac{1-q}{1-p} \\
& +\left[\frac{q}{2}\left(1+\frac{\zeta \sqrt{\Delta t}}{2}\right)-\frac{\nu \sqrt{\Delta t}}{2 \zeta}\right]\left[\log \frac{q}{p}-\frac{1}{\zeta}\left(\frac{\nu}{q}-\frac{\mu}{p}\right) \sqrt{\Delta t}+o(\Delta t)\right]
\end{aligned}
$$

ou seja

$$
\mathrm{E}_{n}^{\mathrm{Q}}\left[\log \frac{\pi_{n}^{\mathrm{Q}}}{\pi_{n}^{\mathrm{P}}}\right]=q \log \frac{q}{p}+(1-q) \log \frac{1-q}{1-p}+\left(\frac{\nu}{\zeta^{2}}-1\right)\left(\frac{\nu}{q}-\frac{\mu}{p}\right) \Delta t+o(\Delta t) .
$$

Vamos agora, por simplicidade, assumir que os dois processos têm drifts iguais, ou seja, $\mu=\nu$, que o prior $\mathbf{P}$ tem volatilidade constante

$$
\sigma_{0}^{2}:=\zeta^{2} p
$$

e que $q$ varia estocasticamente sob $\mathbf{Q}$. Então, definimos a volatilidade instantânea para o Q-processo no instante $t_{n}=n$ por

$$
\sigma^{2}\left(t_{n}\right):=\zeta^{2} q\left(t_{n}\right)
$$

Logo, substituindo as volatilidades e drifts em (3.14) pelas definidas logo acima e desprezando os termos de ordem $\Delta t$, obtemos a seguinte aproximação para a entropia relativa condicional no instante $t_{n}$ :

$$
\eta\left(\sigma^{2}\right):=\frac{\sigma^{2}}{\zeta^{2}} \log \frac{\sigma^{2}}{\sigma_{0}^{2}}+\left(1-\frac{\sigma^{2}}{\zeta^{2}}\right) \log \frac{\zeta^{2}-\sigma^{2}}{\zeta^{2}-\sigma_{0}^{2}} .
$$

Agora, substituindo (3.15) em (3.6) e tomando em consideração uma estimativa para os termos de ordem $\Delta t$, temos

$$
\begin{aligned}
D(\mathbf{P}, \mathbf{Q}) & =\mathbf{E}^{\mathbf{Q}}\left[\sum_{n=0}^{N-1} \eta\left(\sigma^{2}\left(t_{n}\right)\right)+O(\Delta t)\right] \\
& =\frac{1}{\Delta t} \mathbf{E}^{\mathbf{Q}}\left[\sum_{n=0}^{N-1} \eta\left(\sigma^{2}\left(t_{n}\right)\right) \Delta t\right]+O(\Delta t) \\
& =\frac{1}{\Delta t} \mathbf{E}^{\mathbf{Q}}\left[\int_{0}^{T} \eta\left(\sigma^{2}(\tau)\right) d \tau\right]+O(\Delta t)
\end{aligned}
$$

e, consequentemente

$$
D(\mathbf{P}, \mathbf{Q})=\frac{N}{T} \mathbf{E}^{\mathbf{Q}}\left[\int_{0}^{T} \eta\left(\sigma^{2}(\tau)\right) d \tau\right]+O(\Delta t)
$$


onde $T=N \Delta t$ e $\mathbf{E}^{\mathbf{Q}}$ é a esperança com relação a distribuição de probabilidade associada ao processo em tempo contínuo (3.2). A quantidade teórico informacional relevante para $\Delta t \ll 1$ é, portanto

$$
\frac{1}{T} \mathbf{E}^{\mathbf{Q}} \int_{0}^{T} \eta\left(\sigma^{2}(\tau)\right) d \tau
$$

a qual representa a entropia relativa por unidade de tempo de $\mathbf{Q}$ com relação a P.

É fácil ver a pela dedução acima que a entropia relativa por unidade de tempo de $\mathbf{Q}$ com relação a $\mathbf{P}$ ou, mais precisamente, $\eta\left(\sigma^{2}\right)$ não é inerente aos processos (3.1) e (3.2), mas que depende essencialmente da discretização desses processos e, consequentemente, das sequências de aproximação $\left(\mathbf{P}_{N}, \mathbf{Q}_{N}\right)$ usadas para aproximar o par $(\mathbf{P}, \mathbf{Q})$. Dessa maneira, não há unicidade com relação a $\eta$ e para ilustrar esse fato consideremos, por exemplo, notemos que a função $\eta$ em (3.15) depende da constante $\zeta$. Suponhamos, por exemplo, que $\zeta$ assuma valores muito altos, então, podemos aproximar a função $\eta$ em (3.15) por

$$
\eta\left(\sigma^{2}\right) \approx \frac{1}{\zeta^{2}}\left[\sigma^{2} \log \frac{\sigma^{2}}{\sigma_{0}^{2}}-\sigma^{2}+\sigma_{0}^{2}\right] .
$$

De fato, usando série de Taylor, temos

$$
\begin{aligned}
\log \frac{\zeta^{2}-\sigma^{2}}{\zeta^{2}-\sigma_{0}^{2}} & =\log \frac{\zeta\left(1-\frac{\sigma^{2}}{\zeta^{2}}\right)}{\zeta\left(1-\frac{\sigma_{0}^{2}}{\zeta^{2}}\right)} \\
& =\log \left(1-\frac{\sigma^{2}}{\zeta^{2}}\right)-\log \left(1-\frac{\sigma_{0}^{2}}{\zeta^{2}}\right) \\
& =-\frac{\sigma^{2}}{\zeta^{2}}+\frac{\sigma_{0}^{2}}{\zeta^{2}}+O\left(\frac{\sigma^{2}}{\zeta^{2}}\right)+O\left(\frac{\sigma_{0}^{2}}{\zeta^{2}}\right)
\end{aligned}
$$

e, portanto, aproximando $1-\frac{\sigma^{2}}{\zeta^{2}}$ por 1 e substituindo essas aproximações na fórmula (3.15) obtemos a aproximação para $\eta$ sugerida acima. Logo, poderíamos escolher minimizar o funcional (3.17) com

$$
\eta\left(\sigma^{2}\right)=\sigma^{2} \log \left(\frac{\sigma^{2}}{\sigma_{0}^{2}}\right)-\sigma^{2}+\sigma_{0}^{2}
$$

\subsection{Problema de Controle Estocástico}

Devido à não unicidade de $\eta$, desenvolveremos um ambiente para a otimização do funcional:

$$
\frac{1}{T} \mathbf{E}^{\mathbf{Q}}\left[\int_{0}^{T} \eta\left(\sigma^{2}(t)\right) d t\right]
$$


no qual $\eta\left(\sigma^{2}\right)$ pertence a uma classe geral de funções que inclui os casos apresentados na última seção.

Definição 14 Uma função densidade de entropia $\eta\left(\sigma^{2}\right)$ com 'prior' $\sigma_{0}$ é uma função diferenciável a valores reais definida em $0<\sigma^{2}<\infty$ que satisfaz:

1. $0 \leq \eta\left(\sigma^{2}\right)<\infty$;

2. $\eta\left(\sigma^{2}\right)$ é estritamente convexa;

3. $\eta\left(\sigma^{2}\right)$ atinge seu mínimo em $\sigma_{0}^{2}$, ou seja, $\eta\left(\sigma_{0}^{2}\right) \equiv 0$.

Note que as funções (3.15) e (3.18) são exemplos de funções densidade de entropia. Provavelmente, a função densidade de entropia mais simples com prior $\sigma_{0}^{2}$ é a quadrática

$$
\eta\left(\sigma^{2}\right)=\frac{1}{2}\left(\sigma^{2}-\sigma_{0}^{2}\right)^{2}
$$

onde $\sigma^{2} \geq 0$

Para modelar a minimização da entropia relativa no caso em que o tempo é contínuo, consideraremos o seguinte problema de otimização:

Dada uma função densidade de entropia $\eta$ minimizar:

$$
\mathbf{E}^{\mathbf{Q}}\left[\int_{0}^{T} e^{-r \tau} \eta\left(\sigma^{2}(s)\right) d s\right]
$$

sujeito a:

$$
\mathbf{E}^{\mathbf{Q}}\left[e^{-T_{i} r} G_{i}\left(S_{T_{i}}\right)\right]=C_{i}
$$

para $i=1, \ldots, M$, entre todas as distribuições de probabilidade $\mathbf{Q}$ do processo de Itô da forma:

$$
\frac{d S_{t}}{S_{t}}=\sigma_{t} W_{t}+\mu d t
$$

tal que $\sigma_{t}$ é um processo progressivamente mensurável satisfazendo $0<\sigma_{\min } \leq$ $\sigma_{t} \leq \sigma_{\max }<+\infty$.

Para evitar degenarações, assumimos que existe uma única opção por strike/ maturidade e que existe pelo menos um strike diferente de zero.

\section{obs. 6 Opções com mesmo strike}

Nós não consideramos op̧̧ões de venda e compra com mesmos strike e maturidade, pois seus preços devem estar perfeitamente relacionados pela paridade put-call na ausência de arbitragem. 
A restrição imposta sobre $\sigma_{t}$, isto é, $0<\sigma_{\min } \leq \sigma_{t} \leq \sigma_{\max }, 0 \leq t \leq T$ onde $\sigma_{\min }$ e $\sigma_{\max }$ são constantes positivas, é feita por razões técnicas. ${ }^{2}$ Do ponto de vista financeiro, especificar limitantes a priori sobre a volatilidade serve para incorporar no modelo crenças dos agentes em relação às volatilidades extremas.

Para resolver o problema de otimização acima definamos, para $i=1, \ldots, M$, $g_{i}\left(\sigma^{2}\right):=\mathbf{E}^{\mathbf{Q}(\sigma)}\left[e^{-r T_{i}} G_{i}\left(S_{T_{i}}\right)\right]-C_{i}$, de maneira que o problema (3.21) toma a forma:

Minimizar sobre $\sigma^{2}$

$$
\mathbf{E}^{\mathbf{Q}^{\sigma}} \int_{0}^{T} e^{-r \tau} \eta\left(\sigma^{2}(\tau)\right) d \tau
$$

sujeito $a$ :

$$
g_{i}\left(\sigma^{2}\right)=0
$$

Ora, para resolver esse problema vamos utilizar o método de Lagrange, ou seja, resolver o programa de otimização equivale a encontrar os pontos críticos do Lagrangeano

$$
\mathcal{L}\left(\sigma^{2} ; \lambda_{1}, \ldots, \lambda_{M}\right):=\mathbf{E}^{\mathrm{Q}^{\sigma}} \int_{0}^{T} e^{-r \tau} \eta\left(\sigma^{2}(\tau)\right) d \tau+\sum_{i=1}^{M} \lambda_{i} g_{i}\left(\sigma^{2}\right)
$$

ou mais explicitamente

$$
\begin{aligned}
& \mathcal{L}\left(\sigma^{2} ; \lambda_{1}, \ldots, \lambda_{M}\right)= \\
& \quad=\mathbf{E}_{n}^{\mathrm{Q}^{\sigma}} \int_{0}^{T} e^{-r \tau} \eta\left(\sigma^{2}(\tau)\right) d \tau+\sum_{i=1}^{M} \lambda_{i}\left(\mathbf{E}^{\mathbf{Q}^{\sigma}}\left[e^{-r T_{i}} G_{i}\left(S_{T_{i}}\right)\right]-C_{i}\right)
\end{aligned}
$$

e reagrupando os termos da igualdade acima, temos

$$
\begin{aligned}
& \mathcal{L}\left(\sigma^{2} ; \lambda_{1}, \ldots, \lambda_{M}\right)= \\
& \quad=\mathbf{E}^{\mathrm{Q}^{\sigma}}\left[\int_{0}^{T} e^{-r \tau} \eta\left(\sigma^{2}(\tau)\right) d \tau+\sum_{i=1}^{M} \lambda_{i} e^{-r T_{i}} G_{i}\left(S_{T_{i}}\right)\right]-\sum_{i=1}^{M} \lambda_{i} C_{i} .
\end{aligned}
$$

Podemos, então, dividir o problema acima em duas partes. Em primeiro lugar, determinar o $\sigma^{2}$-ponto crítico, $\left(\sigma^{2}\right)^{*}$, da função

$$
\begin{aligned}
& J\left(\sigma^{2} ; \lambda_{1}, \ldots, \lambda_{M}\right):= \\
& :=\mathbf{E}^{\mathbf{Q}^{\sigma}} \int_{0}^{T}\left[e^{-r \tau} \eta\left(\sigma^{2}(\tau)\right)+\sum_{i=1}^{M} \lambda_{i} \delta_{i}(\tau) e^{-r \tau} G_{i}\left(S_{\tau}\right)\right] d \tau
\end{aligned}
$$

\footnotetext{
${ }^{2}$ Essa hipótese garante que a classe de difusões consideradas no problema de controle é fechada com relação a topologia de convergência fraca das medidas sobre caminhos contínuos. É equivalente a parabolicidade uniforme da equação de Hamilton-Jacobi-Bellman e é uma característica desejável para a obtenção de estabilidade em esquemas padrões de diferença finita.
} 
onde $\delta_{i}(\tau):=\delta\left(\tau-T_{i}\right)$ e, então determinar o $\lambda$-ponto crítico de

$$
\mathcal{L}\left(\left(\sigma^{2}\right)^{*} ; \lambda_{1}, \ldots, \lambda_{M}\right)=J\left(\left(\sigma^{2}\right)^{*} ; \lambda_{1}, \ldots, \lambda_{M}\right)-\sum_{i=1}^{M} \lambda_{i} C_{i} .
$$

Agora, suponhamos que o preço inicial do ativo seja $S_{0}$ e que, além disso, $\mathcal{F}_{0}=\{\Omega$,$\} , de modo que$

$$
\mathbf{E}_{0}[X]=\mathbf{E}\left[X \mid \mathcal{F}_{0}\right]=\mathbf{E}[X]
$$

para qualquer variável aleatória $X$. Então, podemos reescrever (3.25) como

$$
\begin{aligned}
& J\left(\sigma^{2} ; \lambda_{1}, \ldots, \lambda_{M}\right)= \\
& \quad=\mathbf{E}_{0, S_{0}}^{\mathbf{Q}^{\sigma}} \int_{0}^{T}\left[e^{-r \tau} \eta\left(\sigma^{2}(\tau)\right)+\sum_{i=1}^{M} \lambda_{i} \delta_{i}(\tau) e^{-r \tau} G_{i}\left(S_{\tau}\right)\right] d \tau .
\end{aligned}
$$

Observe também que determinar o $\sigma^{2}(t)$ que minimiza (3.27) é um caso particular do problema de determinar o processo que minimiza

$$
\begin{aligned}
& J\left(t, S, \sigma^{2} ; \lambda_{1}, \ldots, \lambda_{M}\right)= \\
& =\mathbf{E}_{t, S}^{\mathbf{Q}^{\sigma}} \int_{t}^{T}\left[e^{-r \tau} \eta\left(\sigma^{2}(\tau)\right)+\sum_{i=1}^{M} \lambda_{i} \delta_{i}(\tau) e^{-r \tau} G_{i}\left(S_{\tau}\right)\right] d \tau .
\end{aligned}
$$

para todo $t \in[0, T]$ e onde $S=S(t)$. Passemos, então, a analisar o problema:

Minimizar com relação a $\sigma^{2}$

$$
J\left(t, S, \sigma^{2} ; \lambda_{1}, \ldots, \lambda_{M}\right) .
$$

Para isso lembremos que nosso processo de preços é dado por

$$
\frac{d S(t)}{S(t)}=\mu d t+\sigma(t) d Z(t) .
$$

Então, aplicando o Lema de Itô a $e^{-r t} W(t, S(t))$, obtemos

$$
\begin{aligned}
d\left(e^{-r t} W\right) & =\left(-r e^{-r t} W+e^{-r t} W_{t}\right) d t+e^{-r t} W_{S} d S(t)+\frac{1}{2} e^{-r t} W_{S S}(d S(t))^{2} \\
& =e^{-r t}\left\{W_{S} d S(t)+\left[-r W+W_{t}+\frac{1}{2} \sigma^{2}(t) S^{2}(t) W_{S S}\right] d t\right\}
\end{aligned}
$$

onde $W=W(t, S(t))$.

Antes de seguirmos com os cálculos, definamos o que é um fluxo entrópico.

Definição 15 O fluxo entrópico associado a uma função densidade de entropia $\eta$ é dado por

$$
\Psi(X):=\inf _{\sigma_{\min }^{2} \leq \sigma^{2} \leq \sigma_{\max }^{2}}\left[X \sigma^{2}+\eta\left(\sigma^{2}\right)\right]
$$

para $X \in \mathbb{R}$. 
Então,

$$
\begin{aligned}
\frac{1}{2} \sigma^{2}(t) S^{2}(t) W_{S S} & =\left[\frac{1}{2} S^{2}(t) W_{S S} \sigma^{2}(t)+\eta\left(\sigma^{2}\right)\right]-\eta\left(\sigma^{2}\right) \\
& \geq \Psi\left(\frac{1}{2} S^{2}(t) W_{S S}\right)-\eta\left(\sigma^{2}\right)
\end{aligned}
$$

Portanto,

$$
\begin{aligned}
d\left(e^{-r t} W\right) \geq & e^{-r t}\left\{W_{S} d S(t)+\left[-r W+W_{t}+\Psi\left(\frac{1}{2} S^{2}(t) W_{S S}\right)-\eta\left(\sigma^{2}(t)\right)\right] d t\right\} \\
= & e^{-r t} W_{S}(\mu S(t) d t+\sigma(t) S(t) d Z(t)) \\
& +e^{-r t}\left[-r W+W_{t}+\Psi\left(\frac{1}{2} S^{2}(t) W_{S S}\right)\right] d t-e^{-r t} \eta\left(\sigma^{2}(t)\right) d t \\
= & -e^{-r t} \eta\left(\sigma^{2}(t)\right) d t+e^{-r t} \sigma(t) S(t) W_{S} d Z(t) \\
& +e^{-r t}\left[W_{t}+\Psi\left(\frac{1}{2} S^{2}(t) W_{S S}\right)+\mu S(t) W_{S}-r W\right] d t .
\end{aligned}
$$

Logo, se $W$ é solução da equação diferencial parcial

$$
W_{t}+\Psi\left(\frac{1}{2} S^{2}(t) W_{S S}\right)+\mu S(t) W_{S}-r W=-\sum_{i=1}^{M} \lambda_{i} \delta_{i}(t) G_{i}(S(t))
$$

segue que

$$
d\left(e^{-r t} W\right) \geq-e^{-r t} \eta\left(\sigma^{2}(t)\right) d t+e^{-r t} \sigma(t) S(t) W_{S} d Z(t)-e^{-r t} \sum_{i=1}^{M} \lambda_{i} \delta_{i}(t) G_{i}(S(t))
$$

Agora, integrando a desigualdade acima e tomando a esperança condicional com relação a informação no tempo $t$, obtemos:

$$
\begin{aligned}
\mathrm{E}_{t}^{\mathrm{Q}^{\sigma}}\left[e^{-r T}\right. & \left.W(T, S(T))-e^{-r t} W(t, S(t))\right] \\
& \geq \mathrm{E}_{t}^{\mathbf{Q}^{\sigma}} \int_{t}^{T} e^{-r \tau}\left(-\eta\left(\sigma^{2}(\tau)\right)-\sum_{i=1}^{M} \lambda_{i} \delta_{i}(\tau) G_{i}(S(\tau))\right) d \tau
\end{aligned}
$$

e assumindo $W(T, S(T))=0$, temos

$$
-e^{-r t} W(t, S(t)) \geq-\mathbf{E}_{t}^{\mathbf{Q}^{\sigma}} \int_{t}^{T} e^{-r \tau}\left(\eta\left(\sigma^{2}(\tau)\right)+\sum_{i=1}^{M} \lambda_{i} \delta_{i}(\tau) G_{i}(S(\tau))\right) d \tau
$$

ou seja,

$$
W(t, S(t)) \leq \mathbf{E}_{t}^{\mathbf{Q}^{\sigma}} \int_{t}^{T} e^{-r(\tau-t)}\left(\eta\left(\sigma^{2}(\tau)\right)+\sum_{i=1}^{M} \lambda_{i} \delta_{i}(\tau) G_{i}(S(\tau))\right) d \tau .
$$


Logo, como a desigualdade acima vale para qualquer $\sigma^{2}, W$ é um limitante inferior do membro a direita na equação acima quando variamos $\sigma^{2}$. A questão agora é saber se e quando essa mesma desigualdade é, de fato, uma igualdade. Ora, evidentemente isso ocorre quando temos a igualdade na equação (3.31), isto é, para $\left(\sigma^{2}\right)^{*}(t)$ tal que

$$
\left(\sigma^{2}\right)^{*}(t)=\operatorname{argmin}_{\sigma_{\min }^{2} \leq \sigma^{2} \leq \sigma_{\max }^{2}} \hat{\Psi}\left(\frac{1}{2} S^{2}(t) W_{S S}\right)
$$

onde $\hat{\Psi}(X):=X \sigma^{2}+\eta\left(\sigma^{2}\right)$. Então,

$$
\hat{\Psi}\left(\frac{1}{2} S^{2}(t) W_{S S}\right)=\frac{1}{2} S^{2}(t) W_{S S} \sigma^{2}+\eta\left(\sigma^{2}\right)
$$

Como $\eta$ é, por hipótese, estritamente convexa segue que $\hat{\Psi}$ também é estritamente convexa na variável $\sigma^{2}$, logo, o programa de otimização em (3.33) está bem-definido, ou seja, temos existência e unicidade de solução garantidos. Então, fixando a variável independente em (3.34), derivando em relação a $\sigma^{2}$ e igualando a zero, obtemos

$$
\left(\sigma^{2}\right)^{*}(t)=\left(\eta^{\prime}\right)^{-1}\left(-\frac{1}{2} S^{2}(t) W_{S S}\right) .
$$

Portanto, a solução da equação diferencial parcial (3.31), $W=W(t, S(t))$ realiza, para $\sigma^{2}(t)=\left(\sigma^{2}\right)^{*}(t)$, o ínfimo de $J\left(t, S(t) ; \lambda, \sigma^{2}\right)$, isto é,

$$
W(t, S(t))=\inf _{\sigma_{\min }^{2} \leq \sigma^{2} \leq \sigma_{\max }^{2}} J\left(t, S(t) ; \lambda, \sigma^{2}\right)
$$

e, consequentemente, o problema (3.29) está resolvido. Resumindo todo o argumento acima temos o seguinte teorema

Teorema 23 Fixado um vetor $\left(\lambda_{1}, \ldots, \lambda_{M}\right)$ em $\mathbb{R}^{M}$, seja $W=W(t, S)=$ $W\left(t, S ; \lambda_{1}, \ldots, \lambda_{M}\right)$ solução da equação diferencial parcial

$$
W_{t}+\Psi\left(\frac{1}{2} S^{2}(t) W_{S S}\right)+\mu S(t) W_{S}-r W=-\sum_{i=1}^{M} \lambda_{i} \delta_{i}(t) G_{i}(S(t))
$$

onde $\Psi(X):=\inf _{\sigma_{\text {min }}^{2} \leq \sigma^{2} \leq \sigma_{\text {max }}^{2}}\left[X \sigma^{2}+\eta\left(\sigma^{2}\right)\right], \delta_{i}(t)=\delta\left(T_{i}-t\right), S>0, t \leq T$ com condição final $W(T, \bar{S}) \stackrel{\text { max }}{=}$. Para cada processo $\sigma^{2}(t)$ satisfazendo $\sigma_{\min }^{2} \leq$ $\sigma^{2} \leq \sigma_{m a x}^{2}$ seja $\mathbf{Q}^{\sigma}(t)$ a medida de probabilidade associada ao processo

$$
\frac{d S(t)}{S(t)}=\mu d t+\sigma(t) d Z(t)
$$

então

$$
\begin{aligned}
& W(t, S(t))= \\
& \quad=\inf _{\sigma_{\min }^{2} \leq \sigma^{2} \leq \sigma_{\max }^{2}} \mathbf{E}_{t, S}^{\mathrm{Q}^{\sigma}} \int_{t}^{T} e^{-r(\tau-t)}\left(\eta\left(\sigma^{2}(\tau)\right)+\sum_{i=1}^{M} \lambda_{i} \delta_{i}(\tau) G_{i}\left(S_{\tau}\right)\right) d \tau
\end{aligned}
$$


onde $\mathbf{E}_{t, S}^{\mathbf{Q}^{\sigma^{2}}}$ é a esperança condicionada a informação no tempo $t$ e $S=S(t)$. Além disso, o ínfimo em (3.39) é realizado pelo processo difusivo

$$
\frac{d S(t)}{S(t)}=\mu d t+\sigma^{*}(t) d Z(t)
$$

com

$$
\left(\sigma^{2}\right)^{*}(t, S)=\left(\eta^{\prime}\right)^{-1}\left(-\frac{1}{2} S^{2} W_{S S}\right)
$$

Ora, então o problema em (3.26) passa a ser encontrar o $\lambda$-ponto crítico de

$$
-\sum_{i=1}^{M} \lambda_{i} C_{i}+W(t, S ; \lambda) .
$$

Porém, antes de analisarmos essa questão consideremos o seguinte lema sobre fluxos entrópicos.

Lema 7 Se $\sigma_{\max }<\infty$, então as seguintes afirmações a respeito de $\Psi(X)=$ $\inf _{\sigma_{m i n}^{2} \leq \sigma^{2} \leq \sigma_{m a x}^{2}}\left[X \sigma^{2}+\eta\left(\sigma^{2}\right)\right]$ são verdadeiras:

1. $\Psi(X)$ é côncava em $X$;

2. $\Psi(0)=0$;

3. $\Psi$ é diferenciável;

4. $\Psi^{\prime}(0)=\sigma_{0}^{2}$;

5. $\Psi^{\prime}(X)=\sigma_{\max }$, se $X \leq-\eta^{\prime}\left(\sigma_{\max }\right)$;

6. $\Psi^{\prime}(X)=\sigma_{\min }$, se $X \geq-\eta^{\prime}\left(\sigma_{\min }\right)$.

Prova. Demonstremos em primeiro lugar a concavidade de $\Psi$. Sejam $X$ e $Y$ números reais e $\gamma \in(0,1)$, então

$$
\begin{aligned}
\Psi(\gamma X+(1-\gamma) Y)= & \inf _{\sigma_{\min }^{2} \leq \sigma^{2} \leq \sigma_{\max }^{2}}\left[\gamma X \sigma^{2}+(1-\gamma) Y \sigma^{2}+\eta\left(\sigma^{2}\right)\right] \\
= & \inf _{\sigma_{\min }^{2} \leq \sigma^{2} \leq \sigma_{\max }^{2}}\left[\gamma\left(X \sigma^{2}+\eta\left(\sigma^{2}\right)\right)+(1-\gamma)\left(Y \sigma^{2}+\eta\left(\sigma^{2}\right)\right)\right] \\
\geq & \gamma \inf _{\sigma_{\min }^{2} \leq \sigma^{2} \leq \sigma_{\max }^{2}}\left[X \sigma^{2}+\eta\left(\sigma^{2}\right)\right] \\
& +(1-\gamma) \inf _{\sigma_{\min }^{2} \leq \sigma^{2} \leq \sigma_{\text {max }}^{2}}\left[Y \sigma^{2}+\eta\left(\sigma^{2}\right)\right]
\end{aligned}
$$

ou seja,

$$
\Psi(\gamma X+(1-\gamma) Y) \geq \gamma \Psi(X)+(1-\gamma) \Psi(Y)
$$

o que mostra a concavidade de $\Psi$. 
O segundo item do lema é trivial. De fato,

$$
\Psi(0)=\inf _{\sigma_{\min }^{2} \leq \sigma^{2} \leq \sigma_{\text {max }}^{2}} \eta\left(\sigma^{2}\right)=0
$$

por definição.

Vamos agora mostrar a diferenciabilidade de $\Psi$. Para todo $X \in \mathbb{R}$, seja $\alpha:=\alpha(X) \in\left[\sigma_{\min }, \sigma_{m a x}\right]$ tal que $\Psi(X)=\alpha(X) X+\eta(\alpha(X))$, ou seja, $\alpha$ é tal que para cada $X$ fixado o ínfimo de $\hat{\Psi}_{X}\left(\sigma^{2}\right):=\sigma^{2} X+\eta\left(\sigma^{2}\right)$ quando variamos $\sigma^{2}$ no intervalo $\left[\sigma_{\text {min }}^{2}, \sigma_{\text {max }}^{2}\right]$ é realizado por $\alpha(X)$. Então, dado $\varepsilon \in \mathbb{R}$ qualquer, temos

$$
\begin{aligned}
\frac{\Psi(X+\varepsilon)-\Psi(X)}{\varepsilon}= & \frac{\alpha(X+\varepsilon)(X+\varepsilon)+\eta(\alpha(X-\varepsilon))-\alpha(X) X-\eta(\alpha(X))}{\varepsilon} \\
= & \frac{\alpha(X+\varepsilon)-\alpha(X)}{\varepsilon} X+\frac{\eta \circ \alpha(X+\varepsilon)-\eta \circ \alpha(X)}{\varepsilon} \\
& +\alpha(X+\varepsilon)
\end{aligned}
$$

portanto, $\Psi$ é diferenciável se, $\alpha$ e $\eta \circ \alpha$ são diferenciáveis. Por definição, $\eta$ é diferenciável. Logo, para que $\Psi$ seja diferenciável, basta mostrarmos que $\alpha$ é diferenciável. Para isso, esqueçamos por um instante a restrição $\sigma_{\min }^{2} \leq \sigma^{2} \leq$ $\sigma_{\text {max }}^{2}$ e suponhamos que $\alpha$ pode atingir qualquer número real. Nesse caso, $\alpha(X)$ deve satisfazer

$$
\hat{\Psi}_{X}^{\prime}(\alpha(X))=0
$$

ou seja, $X+\eta^{\prime}(\alpha(X))=0$ ou, equivalentemente

$$
\alpha(X)=\left(\eta^{\prime}\right)^{-1}(-X) .
$$

Desse modo, temos três casos para analisar:

1. se $X$ é tal que $\left(\eta^{\prime}\right)^{-1}(-X) \in\left(\sigma_{\min }^{2}, \sigma_{\max }^{2}\right)$ ou, equivalentemente, se $X \in$ $\left(-\eta^{\prime}\left(\sigma_{\max }^{2}\right),-\eta^{\prime}\left(\sigma_{\min }^{2}\right)\right)$;

2. se $\left(\eta^{\prime}\right)^{-1}(-X) \leq \sigma_{\min }^{2}$ ou, equivalentemente, se $X \geq-\eta^{\prime}\left(\sigma_{\min }^{2}\right)$;

3. se $\left(\eta^{\prime}\right)^{-1}(-X) \geq \sigma_{\max }^{2}$ ou, equivalentemente, se $X \leq-\eta^{\prime}\left(\sigma_{\max }^{2}\right)$.

Devido a convexidade de $\hat{\Psi}$ e a restrição $\sigma^{2} \in\left[\sigma_{\min }^{2}, \sigma_{\max }^{2}\right]$ temos que nos segundo e terceiro casos que $\alpha(X)=\sigma_{\min }^{2}$ e $\alpha(X)=\sigma_{\text {max }}^{2}$, respectivamente, demonstrando assim a diferenciabilidade de $\Psi$ em $\left[-\eta^{\prime}\left(\sigma_{\text {max }}^{2}\right),-\eta^{\prime}\left(\sigma_{\min }^{2}\right)\right]^{C}$. Mais ainda, temos nesses casos

$$
\Psi(X)=\sigma_{\min }^{2} X+\eta\left(\sigma_{\min }^{2}\right)
$$

e

$$
\Psi(X)=\sigma_{\text {max }}^{2} X+\eta\left(\sigma_{\text {max }}^{2}\right)
$$

respectivamente. Logo, para $X \geq-\eta^{\prime}\left(\sigma_{\min }^{2}\right)$ temos temos

$$
\Psi^{\prime}(X)=\sigma_{\min }^{2}
$$


e para $X \leq-\eta^{\prime}\left(\sigma_{\max }^{2}\right)$

$$
\Psi^{\prime}(X)=\sigma_{\max }^{2}
$$

demonstrando assim os itens (5) e (6) do lema.

Vamos analisar agora o que ocorre no intervalo aberto $\left(-\eta^{\prime}\left(\sigma_{\max }^{2}\right),-\eta^{\prime}\left(\sigma_{\min }^{2}\right)\right)$. Como esse intervalo é aberto temos

$$
\alpha(X)=\left(\eta^{\prime}\right)^{-1}(-X)
$$

e como, $\eta^{\prime}$ é invertível e, por hipótese, $\eta$ é diferenciável, segue que $\alpha$ também é diferenciável nesse intervalo. Mais ainda, é possível calcular explicitamente (em função de $\eta$ ) a expressão de sua derivada nesse intervalo. De fato

$$
\begin{aligned}
\alpha^{\prime}(X) & =\frac{d}{d X}\left(\eta^{\prime}\right)^{-1}(-X)=-\left[\left(\eta^{\prime}\right)^{-1}\right]^{\prime}(-X) \\
& =-\frac{1}{\eta^{\prime \prime}\left(\alpha_{X}\right)}
\end{aligned}
$$

onde $\alpha_{X}$ é tal que $\eta^{\prime}\left(\alpha_{X}\right)=-X$, isto é, $\alpha_{X}=\left(\eta^{\prime}\right)^{-1}(-X)$ e, portanto

$$
\alpha^{\prime}(X)=-\frac{1}{\eta^{\prime \prime}\left(\left(\eta^{\prime}\right)^{-1}(-X)\right)}
$$

Então, pela fórmula (3.42), para $X \in\left(-\eta^{\prime}\left(\sigma_{\max }^{2}\right),-\eta^{\prime}\left(\sigma_{\min }^{2}\right)\right)$

$$
\begin{aligned}
\Psi^{\prime}(X) & =\alpha^{\prime}(X)+\eta^{\prime}(\alpha(X)) \alpha^{\prime}(X)+\alpha(X) \\
& =-\frac{X}{\eta^{\prime \prime}\left(\left(\eta^{\prime}\right)^{-1}(-X)\right)}+\frac{X}{\eta^{\prime \prime}\left(\left(\eta^{\prime}\right)^{-1}(-X)\right)}+\left(\eta^{\prime}\right)^{-1}(-X)
\end{aligned}
$$

$\log 0$

$$
\Psi^{\prime}(X)=\left(\eta^{\prime}\right)^{-1}(-X)
$$

Da fórmula acima temos os limites

$$
\begin{aligned}
\lim _{X \rightarrow-\eta^{\prime}\left(\alpha\left(\sigma_{\text {max }}^{2}\right)\right)+} \Psi^{\prime}(X) & =\lim _{X \rightarrow-\eta^{\prime}\left(\alpha\left(\sigma_{\text {max }}^{2}\right)\right)+}\left(\eta^{\prime}\right)^{-1}(-X)=\left(\eta^{\prime}\right)^{-1} \eta^{\prime}\left(\alpha\left(\sigma_{\text {max }}^{2}\right)\right) \\
& =\sigma_{\text {max }}^{2}
\end{aligned}
$$

e

$$
\begin{aligned}
\lim _{X \rightarrow-\eta^{\prime}\left(\alpha\left(\sigma_{\text {min }}^{2}\right)\right)-} \Psi^{\prime}(X) & =\lim _{X \rightarrow-\eta^{\prime}\left(\alpha\left(\sigma_{\min }^{2}\right)\right)-}\left(\eta^{\prime}\right)^{-1}(-X)=\left(\eta^{\prime}\right)^{-1} \eta^{\prime}\left(\alpha\left(\sigma_{\min }^{2}\right)\right) \\
& =\sigma_{\min }^{2}
\end{aligned}
$$

os quais coincidem com os resultados expressos pelas fórmulas (3.45) e (3.44), respectivamente, o que implica a diferenciabilidade de $\Psi$ nos pontos $-\eta^{\prime}\left(\alpha\left(\sigma_{\text {max }}^{2}\right)\right)$ e $-\eta^{\prime}\left(\alpha\left(\sigma_{\max }^{2}\right)\right)$. Consequentemente, temos demonstrado o item (3) do lema. Vamos agora verificar o item (4). Esse segue imediatamente da fórmula (3.48). De fato

$$
\Psi^{\prime}(0)=\left(\eta^{\prime}\right)^{-1}(0)=\sigma_{0}^{2}
$$

como queríamos demonstrar. 
obs. 7 A concavidade estrita de $\Psi$.

Observe que

$$
\Psi^{\prime \prime}(X)=\frac{d}{d X}\left(\eta^{\prime}\right)^{-1}(-X)=-\frac{1}{\eta^{\prime \prime}\left(\left(\eta^{\prime}\right)^{-1}(-X)\right)}<0
$$

pois $\eta^{\prime \prime}$ é positiva uma vez que $\eta$ é estritamente convexa, temos que $\Psi$ é estritamente côncava.

Lembremos que uma equação diferencial parcial não-linear ${ }^{3}$

$$
L\left(t, x, u(t, x), \frac{\partial u(t, x)}{\partial x_{i}}, \frac{\partial^{2} u(t, x)}{x_{i} x_{j}}, \frac{\partial u(t, x)}{\partial t}\right)=0
$$

é parabólica em $D$ com relação a uma solução $u=u(t, x)$ se a função

$$
a(t, x):=\frac{\partial L}{\partial\left(\frac{\partial u}{\partial t}\right)}
$$

é estritamente negativa em D e a matriz $\left(b_{i j}\right)_{i j}$ dada por

$$
b_{i j}:=\frac{\partial^{2} L}{\partial\left(\frac{\partial^{2} u}{\partial x_{i} \partial x_{j}}\right)^{2}}
$$

é não-negativa definida.

Teorema 24 A equação diferencial parcial (3.37) é parabólica.

Prova. De fato, nosso operador diferencial não-linear é dado por

$$
\begin{aligned}
& L\left(t, W, \lambda_{1}, \ldots, \lambda_{M}, W_{S}, W_{\lambda_{i}}, W_{S, \lambda_{i}}, W_{\lambda_{i}, \lambda_{j}}, W_{t}\right) \\
& \quad=-\frac{\partial W}{\partial t}-\Psi\left(\frac{1}{2} S^{2}(t) \frac{\partial^{2} W}{\partial S^{2}}\right)-\mu S(t) \frac{\partial W}{\partial S}+r W-\sum_{i=1}^{M} \lambda_{i} \delta_{i}(t) G_{i}(S(t))
\end{aligned}
$$

para $i, j=1, \ldots M$. Note que os coeficientes de todas derivadas parciais envolvendo $\lambda_{i}$ para algum $i=1, \ldots, M$ é nulo. Evidentemente, nesse caso $a(t, S, \lambda)=$ -1 em todo domínio de definição da solução $W$ de modo que, para terminarmos a demonstração do teorema resta apenas mostrar que a matriz $\left(b_{i j}\right)_{i j}$ definida acima é não-negativa definida. Em primeiro lugar, notemos que

$$
\frac{\partial^{2} L}{\partial\left(\frac{\partial^{2} W}{\partial S \partial \lambda_{i}}\right)^{2}}=\frac{\partial^{2} L}{\partial\left(\frac{\partial^{2} W}{\partial \lambda_{i} \partial S}\right)^{2}}=\frac{\partial^{2} L}{\partial\left(\frac{\partial^{2} W}{\partial \lambda_{i} \partial \lambda j}\right)^{2}}=0
$$

${ }^{3}$ Ver Friedman[8]. 
para todo $i, j=1, \ldots, M$. E, finalmente

$$
\frac{\partial^{2} L}{\partial\left(\frac{\partial^{2} W}{\partial S \partial S}\right)^{2}}=-\frac{1}{4} S^{4} \Psi^{\prime \prime}\left(\frac{1}{2} S^{2} \frac{\partial^{2} W}{\partial S^{2}}\right) \geq 0
$$

pois $\Psi$ é côncava e, portanto, tem segunda derivada não-positiva. Ora, mas então a matriz $\left(b_{i j}\right)_{i j}$ é não-negativa definida e, consequentemente, o operador $L$ é parabólico.

Consideremos então o seguinte resultado sobre diferenciabilidade de soluções de equações diferenciais parabólicas.

Teorema 25 Seja $u(t, x)$ solução da equação (3.49) em $D$ e assumamos que $\frac{\partial L}{\partial x}, \frac{\partial L}{\partial u_{j}}$ são Hölder contínuas (com expoente $\alpha$ ) em $\left(x, t, u_{1}, \ldots, u_{N}\right)$ para $j=$ $1, \ldots, N$ assim como todas derivadas de ordem menor ou igual a $p \geq 0$ com relação a $x, u_{1}, \ldots, u_{N}$ para $(t, x) \in D,-\infty<u_{j}<\infty$ para $j=1, \ldots, N$. Então, se (3.49) é parabólica em $D$ com relação a $u(t, x)$ e se $D_{x} u, D_{x}^{2} u, D_{t} u$ são Hölder contínuas em $D$ com algum expoente $\epsilon>0$, temos que

$$
D_{x}^{m} u
$$

$e$

$$
D_{t} D_{x}^{k} u
$$

existem e são Hölder contínuas (com expoente $\alpha$ ) em $D$ para $0 \leq m \leq p+3 e$ $0 \leq k \leq p+1$.

Então, em consequência da parabolicidade da equação (3.37), a primeira parte da próxima proposição abaixo segue imediatamente do teorema 25 :

Proposição $7^{4}$ A função $W\left(t, S ; \lambda_{1}, \ldots, \lambda_{M}\right)$ é continuamente diferenciável $e$ côncava em $\left(\lambda_{1}, \ldots, \lambda_{M}\right)$.

Prova. Como a diferenciabilidade de $W$ em relação a $\lambda$ é consequência do teorema acima, resta-nos apenas verificar a concavidade de $W$ em relação a $\left(\lambda_{1}, \ldots, \lambda_{M}\right)$. obtemos

Usando a notação $W_{i}:=\frac{\partial W}{\partial \lambda_{i}}$ e derivando a equação (3.37) em relação a $\lambda_{i}$,

$$
W_{i t}+\frac{1}{2} \Psi^{\prime}\left(\frac{1}{2} S^{2}(t) W_{S S}\right) S^{2} W_{i S S}+\mu S(t) W_{i S}-r W_{i}=-\delta_{i}(t) G_{i}(S(t))
$$

${ }^{4}$ Para concluir que $H$ é estritamente côncava em todo seu domínio, isto é, que $H\left(t^{*}, S^{*}\right)<0$ em todo o domínio, basta notar que $H\left(t^{*}, S^{*}\right)<0$ em um conjunto com medida de Lebesgue positiva. $\mathrm{E}$ isso é verdade, pois, $H$ é estritamente negativa no interior do seu domínio (devido a estrita côncavidade de $\Psi$, ver obsevação 7 ). Então, como a solução fundamental de $H$ é estritamente negativa segue que $H\left(t^{*}, S^{*}\right)<0$ em todo o domínio. 
para $i=1, \ldots, M$ com $W_{i}(T, S)=0$. Então, derivando novamente em relação a $\lambda_{j}$ obtemos

$$
\begin{aligned}
W_{i j t} & +\frac{1}{2} \Psi^{\prime}\left(\frac{1}{2} S^{2}(t) W_{S S}\right) S^{2} W_{i j S S} \\
& +\frac{1}{4} \Psi^{\prime \prime}\left(\frac{1}{2} S^{2}(t) W_{S S}\right) S^{4} W_{i S S} W_{j S S}+\mu S(t) W_{i j S}-r W_{i j}=0
\end{aligned}
$$

Seja $H$ a matriz Hessiana de $W$, isto é $H=\left(W_{i j}\right)_{i, j}$. Para mostrarmos que $W$ é côncava em relação a $\lambda=\left(\lambda_{1}, \ldots, \lambda_{M}\right)$ basta demonstrarmos que $H$ é negativa-definida, ou seja, que dado um vetor $\theta \in \mathbb{R}^{M}$ qualquer, então, $H(\theta)=$ $\sum_{i, j=1}^{M} W_{i j} \theta_{i} \theta_{j} \leq 0$. De (3.51) temos que

$$
\begin{aligned}
\sum_{i, j=1}^{M} W_{i j t} \theta_{i} \theta_{j} & +\frac{1}{2} \Psi^{\prime}\left(\frac{1}{2} S^{2}(t) W_{S S}\right) S^{2} \sum_{i, j=1}^{M} W_{i j S S} \theta_{i} \theta_{j} \\
& +\frac{1}{4} \Psi^{\prime \prime}\left(\frac{1}{2} S^{2}(t) W_{S S}\right) S^{4} \sum_{i, j=1}^{M} W_{i S S} W_{j S S} \theta_{i} \theta_{j} \\
& +\mu S(t) \sum_{i, j=1}^{M} W_{i j S} \theta_{i} \theta_{j}-r \sum_{i, j=1}^{M} W_{i j} \theta_{i} \theta_{j}=0
\end{aligned}
$$

e, portanto, observando que $\sum_{i, j=1}^{M} W_{i S S} W_{j S S} \theta_{i} \theta_{j}=\left(\sum_{i, j=1}^{M} W_{i S S} \theta_{i}\right)^{2}$ temos

$$
\begin{aligned}
H_{t} & +\frac{1}{2} \Psi^{\prime}\left(\frac{1}{2} S^{2}(t) W_{S S}\right) S^{2} H_{S S} \\
& +\frac{1}{4} \Psi^{\prime \prime}\left(\frac{1}{2} S^{2}(t) W_{S S}\right) S^{4}\left(\sum_{i, j=1}^{M} W_{i S S} \theta_{i}\right)^{2}+\mu S(t) H_{S}-r H=0 .
\end{aligned}
$$

Agora suponhamos que $H$ atinge seu máximo em $\left(t^{*}, S^{*}\right)$ no interior do seu domínio e que $H\left(t^{*}, S^{*}\right)>0$. Então,

$$
H_{t}\left(t^{*}, S^{*}\right)=H_{S}\left(t^{*}, S^{*}\right)=0
$$

portanto, nesse ponto temos

$$
\begin{aligned}
& \frac{1}{2} \Psi^{\prime}\left(\frac{1}{2}\left(S^{*}\right)^{2} W_{S S}\left(t^{*}, S^{*}\right)\right) S^{2} H_{S S}\left(t^{*}, S^{*}\right) \\
& \quad+\frac{1}{4} \Psi^{\prime \prime}\left(\frac{1}{2}\left(S^{*}\right)^{2} W_{S S}\left(t^{*}, S^{*}\right)\right)\left(S^{*}\right)^{4}\left(\sum_{i, j=1}^{M} W_{i S S}\left(t^{*}, S^{*}\right) \theta_{i}\right)^{2} \\
& \quad-r H\left(t^{*}, S^{*}\right)<0
\end{aligned}
$$


devido a concavidade de $\Psi$ e pelo fato que $\Psi^{\prime}(X) \geq \sigma_{\min }>0$. Mas isso contradiz (3.52). Portanto, devemos ter

$$
H\left(t^{*}, S^{*}\right) \leq 0
$$

para todo ponto interior (em relação ao domínio de $W$ ). Por continuidade,

$$
H\left(t^{*}, S^{*}\right) \leq 0
$$

em todo seu domínio. Logo, $W$ deve ser côncava.

Ora, como vimos na demonstração acima, derivando a equação (3.37) com relação a $\lambda$ obtemos as equações diferenciais parciais

$$
W_{i t}+\frac{1}{2} \Psi^{\prime}\left(\frac{1}{2} S^{2}(t) W_{S S}\right) S^{2} W_{i S S}+\mu S(t) W_{i S}-r W_{i}=-\delta_{i}(t) G_{i}(S(t))
$$

para $i=1, \ldots, M$ com $W_{i}(T, S)=0$. Essas equações podem ser interpretadas como equações de apreçamento para as $M$ opções usando o processo difusivo com volatilidade

$$
\left(\sigma^{2}\right)^{*}(t, S)=\left(\eta^{\prime}\right)^{-1}\left(-\frac{1}{2} S^{2} W_{S S}\right)
$$

Em particular, o modelo estará calibrado se

$$
W_{i}(0, S)=C_{i}
$$

ou, equivalentemente, se

$$
\frac{\partial}{\partial \lambda_{i}} W(0, S ; \lambda)=C_{i}
$$

Isso mostra que a calibração é equivalente a minimizar a função $W(0, S ; \lambda)$ $\sum \lambda_{i} C_{i}$. A proposição abaixo formaliza essa afirmação e mostra que a escolha da volatilidade resolve o problema de controle estocástico.

\section{Proposição 8 Defina}

$$
V\left(t, S ; \lambda_{1}, \ldots, \lambda_{M}\right):=W\left(t, S ; \lambda_{1}, \ldots, \lambda_{M}\right)-\sum_{i=1}^{M} \lambda_{i} C_{i}
$$

e suponha que, para $S$ fixo, $V\left(0, S ; \lambda_{1}, \ldots, \lambda_{M}\right)$ atinge um máximo global no ponto $\left(\lambda_{1}^{*}, \ldots, \lambda_{M}^{*}\right)$ em $\mathbb{R}^{M}$. Então, a classe de medidas de probabilidade que satisfazem as restrições de preço e os limites de volatilidade é não-vazia e o problema de controle estocástico admite uma única solução. A solução corresponde ao processo difusivo com volatilidade

$$
\left(\sigma^{2}\right)^{*}(t, S)=\left(\eta^{\prime}\right)^{-1}\left(-\frac{1}{2} S^{2} W_{S S}\right) .
$$

para $0 \leq t \leq T$. 
Prova. Observe que

$$
V\left(0, S ; \lambda_{1}, \ldots, \lambda_{M}\right)=\inf _{\sigma_{\min }^{2} \leq \sigma^{2} \leq \sigma_{\max }^{2}}\left(a\left(\sigma^{2}\right)+\sum_{i=1}^{M} \lambda_{i} b_{i}\left(\sigma^{2}\right)\right)
$$

onde

$$
a\left(\sigma^{2}\right)=\mathbf{E}^{\mathbf{Q}^{\sigma}} \int_{0}^{T} e^{-r \tau} \eta\left(\sigma^{2}(\tau)\right) d \tau
$$

$\mathrm{e}$

$$
b_{i}\left(\sigma^{2}\right)=\mathbf{E}^{\mathrm{Q}^{\sigma}}\left[e^{-r T_{i}} G_{i}\left(S_{T_{i}}\right)\right]-C_{i}
$$

para $i=1, \ldots, M$. Seja $\left(\sigma^{2}\right)^{*}$ a volatilidade ótima que realiza o ínfimo em (3.55) (cuja existência e unicidade estão garantidos pelo teorema 23) para o máximo global $\left(\lambda_{1}^{*}, \ldots, \lambda_{M}^{*}\right)$. Note agora que

$$
a\left(\left(\sigma^{2}\right)^{*}\right)+\sum_{i=1}^{M} \lambda_{i} b_{i}\left(\left(\sigma^{2}\right)^{*}\right)
$$

descreve um híper-plano passando por $\left(\lambda_{1}^{*}, \ldots, \lambda_{M}^{*}\right)$, ou seja, pelo mínimo de $V(0, S)$ e, portanto, para todo $\left(\lambda_{1}, \ldots, \lambda_{M}\right)$ temos $a\left(\left(\sigma^{2}\right)^{*}\right)+\sum_{i=1}^{M} \lambda_{i} b_{i}\left(\left(\sigma^{2}\right)^{*}\right) \leq$ $V\left(0, S ; \lambda_{1}, \ldots, \lambda_{M}\right)$. Além disso, devido a concavidade e diferenciabilidade de $V(0, S)$ (em relação a $\left.\left(\lambda_{1}, \ldots, \lambda_{M}\right)\right)$ temos que esse híper-plano é tangente a $V(0, S)$ no ponto $\left(\lambda_{1}^{*}, \ldots, \lambda_{M}^{*}\right)$. Consequentemente,

$$
0=\left(\frac{\partial V}{\partial \lambda_{i}}\right)_{\lambda=\lambda^{*}}=b_{i}\left(\left(\sigma^{*}\right)^{2}\right)=\mathbf{E}^{\mathbf{Q}^{\sigma^{*}}}\left[e^{-r T_{i}} G_{i}\left(S_{T_{i}}\right)\right]-C_{i}
$$

para $i=1, \ldots, m$. Logo, a classe de medidas de probabilidade que satisfazem as restrições de preço e os limites de volatilidade é não-vazia, pois, $\mathrm{Q}^{\left(\sigma^{2}\right)^{*}}$ pertence a essa classe.

Vamos agora mostrar que dentre todos elementos da classe acima $\mathrm{Q}^{\left(\sigma^{2}\right)^{*}}$ é aquele que minimiza a entropia relativa. De fato, suponha que $\hat{\sigma}^{2}$ cuja medida de probabilidade associada pertence a classe de medidas descritas no teorema. Então,

$$
\mathbf{E}^{\mathbf{Q}^{\tilde{j}}}\left[e^{-r T_{i}} G_{i}\left(S_{T_{i}}\right)\right]=C_{i}
$$

para $i=1, \ldots, M$. Então, $b_{i}\left(\hat{\sigma}^{2}\right)=0$, então

$$
\begin{aligned}
a\left(\hat{\sigma}^{2}\right) & =a\left(\hat{\sigma}^{2}\right) \\
& \geq \inf _{\sigma_{\min }^{2} \leq \sigma^{2} \leq \sigma_{\max }^{2}}\left(a\left(\sigma^{2}\right)+\sum_{i=1}^{M} \lambda_{i} b_{i}\left(\sigma^{2}\right)\right) \\
& =a\left(\left(\sigma^{2}\right)^{*}\right) .
\end{aligned}
$$

Ora, então mostramos que se existir o vetor $\left(\lambda_{1}^{*}, \ldots, \lambda_{M}^{*}\right)$, existe uma solução do problema de otimização que nos propomos resolver. Mais ainda, tal solução é 
única. Consequentemente, uma vez obtida a solução $W$ da equação de HamiltonJacobi-Bellman, é possível determinar a expressão da volatilidade implícita:

$$
\left(\sigma^{2}\right)^{*}(t, S)=\left(\eta^{\prime}\right)^{-1}\left(-\frac{1}{2} S^{2} W_{S S}\right) .
$$

para $0 \leq t \leq T$. Ou seja, obtemos assim a superfície de volatilidade implícita via minimização da entropia relativa. 


\section{Referências Bibliográficas}

[1] AMARI, S., Differential-Geometrical Methods in Statistics. 1a edição. New York, Springer-Verlag, Lecture Notes in Statistics, 28, 1985. 290p.

[2] AVELlanedA, M., FRIEDMAN, C., HOLMES, R. e SAMPERI, D. Calibrating Volatility Surfaces via Relative-Entropy Minimization. Courant Institute, New York University. Working Paper.

[3] COChrane, J. H. Asset Pricing. 1a edição. Princeton, New Jersey, Princeton University Press, 2001. 530p.

[4] COVER, T. M. e THOMAS, J. A., Elements of Information Theory. 1a edição. New York, John Willey \& Sons, 1991.

[5] CSISZÁR, I. I-divergence Geometry of Probability Distributions and Minimization Problems. Ann. Probability, 3: 146-158, 1975.

[6] DUfFIE, D., Dynamic Asset Pricing Theory. 3a edição. Princeton, New Jersey, Princeton University Press, 2001. 465p.

[7] Fleming, W. H. e SONER, H. M., Controlled Markov Processes and Viscosity Solutions. 1a edição. New York, Springer-Verlag, 1992. 428p.

[8] FRIEDMAN, A. Partial Differential Equations of Parabolic Type. 1a edição. Englewood Cliffs, N. J., Prentice Hall, INC., 1964. 347p.

[9] GULKO, B. The Entropy Pricing Theory. Tese de Doutorado, Yale University.

[10] IBRAGIMOV, I. A. e HAS'MINSKII, R. Z. Statistical Estimation Asymptotic Theory. 1a edição. New York, Springer-Verlag, 1981. 403p.

[11] IHARA, S. Information Theory. 1a edição. Singapura, World Scientific Publishing Co., 1993. 308p.

[12] OKSENDAL, B. Stochastic Differential Equations. 5a edição. New York, Springer-Verlag, 2000. 326p.

[13] STUTZER, M. J. Simple Entropic Derivation of a Generalized BlackScholes Option Pricing Model. Entropy, 2, 50-77, 2000. 